$\$ 5.00$

A2.2.1988-407

\title{
Synopsis of the
}

Digenea of
Mammals of
North Amerioa

Murray J. Kennedy

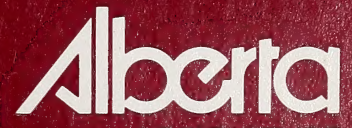


Other titles in this series -

Synopsis of the Parasites of Domesticated Mammals of Canada Murray J. Kennedy and Robert A. Newman, 1986.

Revised edition available summer 1988.

Synopsis of the Parasites of Vertebrates of Canada - Helminths and Protozoa of Terrestrial Mammals Murray J. Kennedy, 1986.

Synopsis of the Parasites of Vertebrates of Canada

- Ectoparasites of Terrestrial Mammals

Murray J. Kennedy and Robert A. Newman, 1986.

Copies of these publications are available from the Queen's Printer, 11510 Kingsway Avenue, Edmonton T5G 2 Y5 Each order must be accompanied by cheque for $\$ 5.00$ made out to The Provincial Treasurer 


\section{Synopsis of the}

Digenea of
Mammals of
North America

Murray J. Kennedy

Alberta Agriculture

Veterinary Laboratory

6909 - 116 Street

Edmonton, Alberta

Canada

T6H 4P2 


\section{ACKNOWLEDGEMENTS}

I am particularly indebted to three people for their help during the preparation of this manuscript. Dr. Rafael Lamothe Argumedo, Instituto de Biologia, Universidad Nacional Autonoma de Mexico, sent me copies of many of the publications on the Digenea of mammals of Mexico; Mr. Robert Kralka helped during the early stages of the manuscript; and Mrs. Darcy Whelihan patiently typed the numerous revisions of the manuscript. I am also indebted to the staff of the Print Media Branch, Alberta Agriculture, for publishing the manuscript. Mr. Andrew Bartley typeset the manuscript, and Mrs. Anne Packer coordinated the printing of the publication. I would also like to thank the members of our library who helped secure copies of many of the papers reviewed for the Synopsis. 


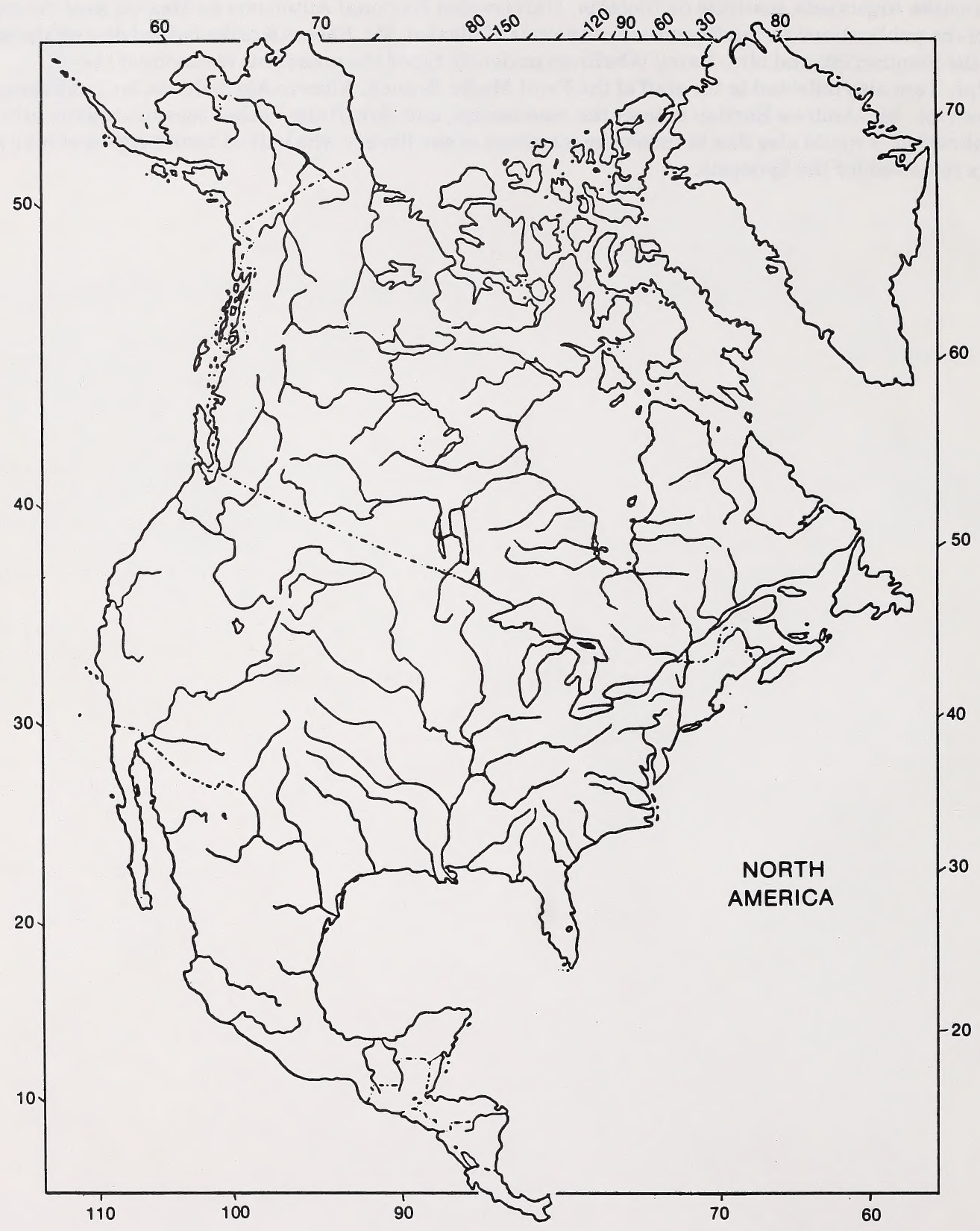

Figure 1. Geographic range of hosts covered in this synopsis. 


\section{INTRODUCTION}

The Digenea of North American mammals are still incompletely known, and this synopsis should not be used as a primary source of zoogeographic information, since the completeness of coverage and the geographical range covered vary from author to author and from group to group. The synopsis includes records from continental North America north of Panama (terrestrial mammals) and adjacent oceans (marine mammals)(Figure 1). Canadian arctic islands and Greenland are included in the coverage because of their interconnection with ice and the wide migration of many mammals. Conversely, oceanic islands such as Hawaii (USA), Guadalupe (Mexico), etc., have not been included because of their distance from continental North America.

The compiled information on the Digenea of North America covers the period from 1858 to June 1987, and was extracted from numerous publications in the primary scientific literature. Journal series such as the Canadian Journal of Zoology, Canadian Journal of Research, Journal of Wildlife Diseases, Proceedings of the Helminthological Society of Washington, and the Journal of Parasitology were searched in their entirety. Abstracting series such as Zoological Records and Helminthological Abstracts were scanned for additional references. Theses and reports of federal and provincial governments and of other institutions were not searched extensively, hence, some existing records may have been omitted.

I conducted a least squares regression analysis on the number of papers published since 1858 (Figure 2) to estimate the number of publications we may expect to see in the $1980 \mathrm{~s}$. The estimate was based on the multivariate general linear model: $y=m x+b$ where $y$ is a vector of dependent variables, $x$ is a vector of independent variables, $b$ is a vector of regression coefficients, and $\mathrm{m}$ is a vector of random errors. The analysis predicted $123 \pm 18$ papers will be published in the 1980 s. In the present synopsis 50 papers up to June 1987 were reviewed.

In providing references to the parasite-host-distribution data, I attempted to include only reports based on original records. A list of publications not providing original parasite-host-distribution records but dealing with some aspect of Digenea in North American mammals has been included in a supplementary list of references.

The synopsis indicates that up to June 1987, at least 214 species of Digenea divided among 28 families have been reported from 138 species of mammals of North America. Parasites and hosts not identified to the species level are not included in the total number. The 138 species of mammals are divided among 11 orders and 35 families.

The taxonomic arrangement adopted for the Digenea for the most part follows that of Brooks et al. (1985)(families and orders) and Schell (1985) (genera). Host nomenclature and classification conforms to that of Hall (1981), except that Odocoileus rather than Dama is used for the white-tailed, mule, and black-tailed deer. The entries in the synopsis are organized in accordance with the format adopted by Margolis and Arthur (1979) in their Synopsis of the Parasites of Fishes of Canada.

In the Parasite-Host list, species, genera, family, and order names are listed alphabetically. For each parasite species listed, the following information is provided:

1. The current scientific name, including author(s) and date(s), followed by any recognized synonyms that may have been used in establishing the North American record(s). Adult and larval stages are recorded separately for the Diplostomidae. No attempt was made to evaluate systematically the validity of published records, but attention is drawn to obvious errors.

2. The site of occurrence of the parasite in its host(s) (aberrant sites usually are not included). When the site was not given, the likely site was determined from other records.

3. The hosts. Only currently accepted scientific names are given in the Parasite-Host List. Numbers in parentheses after each host name correspond with the numbers assigned to the references establishing the particular parasite-host record(s).

4. Distribution (Dist.) Species distribution is indicated by province or territorial boundary (Canada), state (United States) or country (Central America) and are listed in alphabetical order according to the abbreviations listed in Table 1. 
5. Authors responsible for the records are listed in chronological order of the published records. The records are numbered and each reference is followed by the locality or localities from which the parasite was (were) reported. A corresponding number is placed after the host name to indicate the author(s) responsible for particular host-parasite locality records. When only one host is listed for a particular parasite, the references are not numbered, and when all records are from the same locality, the latter was not listed after the authors' names.

6. Under remarks, explanatory comments, as required, are offered on systematics, nomenclature, misidentifications, and synonyms of parasites, and on the hosts, sites within hosts, and validity of previous records.

In the Host-Parasite List, within each order, the families, genera, species, and subspecies are listed alphabetically. Host synonyms included are only those that were used in establishing the North American parasite records. After the name of each parasite species, its geographic distribution in the host in question is given in parentheses.

The References are given in two parts, the first includes those directly referred to in the text, and a second, Supplementary References contains a list of publications dealing with various aspects of Digenea of North America mammals but which do not contain original parasite-host records.

Figure 2 Number of papers published on the Digenea of mammals from 1850 to June, 1987.

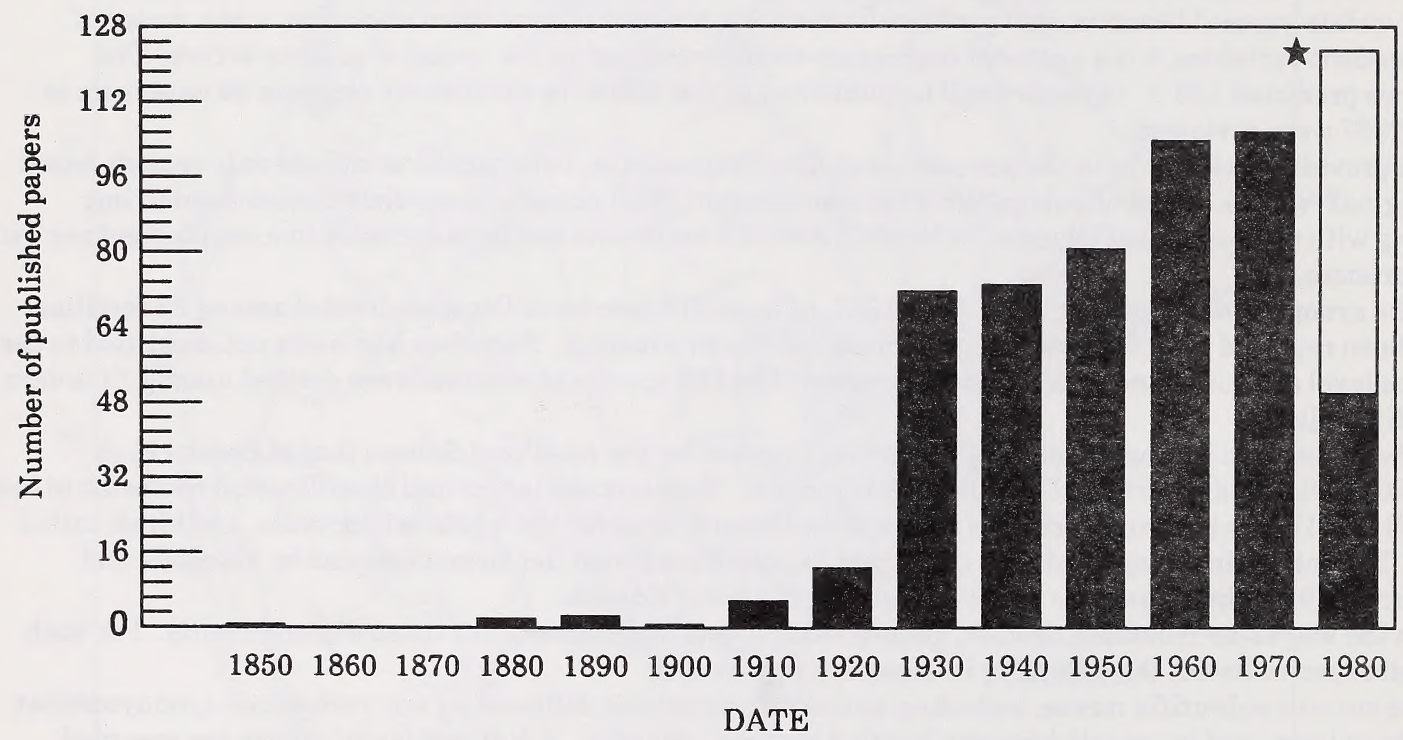

$\star$ Predicted number of papers published by the end of 1989 . 
Table 1. List of abbreviations used for parasite distribution data in the synopsis.

\begin{tabular}{|c|c|c|c|c|}
\hline \multirow{15}{*}{$\begin{array}{l}\text { Country } \\
\text { Canada }\end{array}$} & Province/Territory/ & Abbreviation & Country/State & Abbreviation \\
\hline & Alberta & Alta & Minnesota & MN \\
\hline & British Columbia & $\mathrm{BC}$ & Mississippi & MS \\
\hline & Manitoba & Man & Missouri & MO \\
\hline & New Brunswick & NB & Montana & MT \\
\hline & Newfoundland & Nfld & Nebraska & NB \\
\hline & (including Labrador) & & Nevada & NV \\
\hline & Nova Scotia & NS & New Hampshire & NH \\
\hline & Northwest Territories & NWT & New Jersey & NJ \\
\hline & Ontario & Ont & New Mexico & NM \\
\hline & Prince Edward Island & PEI & New York & NY \\
\hline & Quebec & Que & North Carolina & $\mathrm{NC}$ \\
\hline & Saskatchewan & Sask & North Dakota & ND \\
\hline & Yukon Territories & YT & Ohio & $\mathrm{OH}$ \\
\hline & State & & Oklahoma & OK \\
\hline \multicolumn{3}{|c|}{ United States } & Oregon & OR \\
\hline & Alabama & $\mathrm{AL}$ & Pennsylvania & PA \\
\hline & Alaska & AK & Puerto Rico & PR \\
\hline & Arizona & $\mathrm{AZ}$ & Rhode Island & RI \\
\hline & Arkansas & $\mathrm{AR}$ & South Carolina & $\mathrm{SC}$ \\
\hline & California & $\mathrm{CA}$ & South Dakota & $\mathrm{SD}$ \\
\hline & Colorado & $\mathrm{CO}$ & Tennessee & TN \\
\hline & Connecticut & CT & Texas & $\mathrm{TX}$ \\
\hline & Delaware & $\mathrm{DE}$ & Utah & UT \\
\hline & District of Columbia & $\mathrm{DC}$ & Vermont & VT \\
\hline & Florida & FL & Virginia & VA \\
\hline & Georgia & GA & Washington & WA \\
\hline & Hawaii & HI & West Virginia & WV \\
\hline & Idaho & ID & Wisconsin & WI \\
\hline & Illinois & IL & Wyoming & WY \\
\hline & Indiana & IN & & \\
\hline & Iowa & ID & Mexico & -- \\
\hline & Kansas & $\mathrm{KS}$ & & \\
\hline & Kentucky & KY & (Central America) & \\
\hline & Louisiana & LA & Belize & -- \\
\hline & Maine & ME & Costa Rica & -- \\
\hline & Maryland & MD & El Salvador & -- \\
\hline & Massachusetts & MA & Honduras & -- \\
\hline & Michigan & MI & Nicaragua & -- \\
\hline & & & Panama & -- \\
\hline
\end{tabular}


Digitized by the Internet Archive in 2015 


\section{PARASITE - HOST LIST}




$$
2
$$


CLASS TREMATODA Rudolphi, 1808 SUBCLASS DIGENEA

van Beneden, 1858

ORDER ECHINOSTOMATIFORMES

\section{LaRue, 1957}

FAMILY CAMPULIDAE Odhner, 1926

Campula oblongata Cobbold, 1858

Site: bile duct, pancreatic duct, hepatopancreatic duct, beneath the serous membrane of the liver.

Hosts:Phocoena phocoena $(1,4,5)$

Phocoenoides dalli $(2,3,6)$

Dist.: CA, Nfld, OR, WA

Records: 1. Ching and Robinson 1959 (WA);

2. Dailey 1971 (CA); 3. Migaki et al. 1971

(unspecified); 4. Smith and Threlfall 1973

(Nfld); 5. Dailey and Stroud 1978 (OR); 6.

Dailey and Walker 1978 (CA)

Campula palliata (Looss, 1885)

Syn.: Distomum palliatum Looss, 1885

Site: bile duct, pancreatric duct, liver

Hosts:Delphinus delphis (1)

Steno bredanensis (3)

Tursiops nesarnack (2)

Dist.: Costa Rica, FL

Records: 1. Looss 1885 (Costa Rica); 2.

Woodard et al. 1969 (FL); 3. Forrester and

Robertson 1975 (FL)

Campula rochebruni (Poirier, 1886)

Syn.: Distomum rochebruni Poirier, 1886

Site: bile duct, pancreatic duct, hepatopancreatic duct

Host: Delphinus delphis

Dist.: CA

Records: Ridgway and Dailey 1972; Dailey and Walker 1978

Hadwenius mironovus (Krotov and Deliamure, 1952)

Syn.: Leucasiella mironovi Krotov and

Deliamure, 1952

Site: stomach, duodenum

Host: Phocoena phocoena

Dist.: OR

Record: Dailey and Stroud 1978

Hadwenius nipponicus Yamaguti, 1950

Site: stomach, duodenum

Host: Phocoena phocoena

Dist.: OR, WA
Records: Ching and Robinson 1959 (WA);

Dailey and Stroud 1978 (OR)

Hadwenius seymouri (Price, 1932)

Syn.: Odhneriella seymouri (Price, 1932) Skrjabin, 1944

Site: intestine

Host: Delphinapteras leucas

Dist.: AK

Record: Price 1932

Lecithodesmus goliath (van Beneden, 1858)

Odhner, 1905

Site: bile duct

Host: Balaenoptera physalus (1)

Eschrichtius gibbosus (2)

Dist.: CA, BC

Record: 1. Margolis and Pike 1955 (BC); 2.

Rice and Wolman 1971 (CA)

Lecithodesmus spinosus Margolis and Pike, 1955

Site: bile ducts

Host: Balaenoptera borealis

Dist.: BC

Record: Margolis and Pike 1955

Orthosplanchnus arcticus Odhner, 1905

Site: liver, bile ducts

Hosts:Globicephala melaena $(2,3)$

Phoca hispida (1)

Dist.: Nfld, NWT

Records: 1. Cooper 1921 (NWT); 2. Cowan 1966 (Nfld), 3. 1967 (Nfld)

Remarks: Cowan (1966) initially reported this species simply as "trematode worms."

Orthosplanchnus fraterculus Odhner, 1905

Site: gall bladder, bile duct

Hosts: Enhydra lutris $(1,2)$

Erignathus barbatus (1)

Dist.: AK

Records: 1. Rausch and Locker 1951; 2.

Rausch 1953

Synthesium tursionis (Marchi, 1873)

Site: intestine

Host: Steno bredanensis

Dist.: FL

Record: Forrester and Robertson 1975

Zalophotrema hepaticum Stunkard and Alvey, 1929

Site: bile duct, liver, small intestine

Hosts: Eumetopias jubatus (7)

Tursiops nesarnack (3)

Zalophus californianus $(1,2,4,5,6,7)$

Dist.: CA, NY, OR 
Records: 1. Stunkard and Alvey 1929 (NY); 2. 1930 (NY); 3. Brown et al. 1960 (CA); 4. Fleischman and Squire 1970 (CA); 5.

Sweeney 1974 (CA); 6. Sweeney and Gilmartin 1974 (CA); 7. Stroud and Dailey 1978 (OR)

\section{Zalophotrema spp.}

Site: brain, liver

Hosts:Lagenorhynchus obliquidens (1) Zalophus californianus (2)

Dist.: CA

Records: 1. Ridgway and Dailey 1972; 2.

Schroeder et al. 1973

Campulidae gen. spp.

Site: brain, pancreas

Hosts:Delphinus delphis (1)

Erignathus barbatus (2)

Dist.: AK, CA

Records: 1. Ridgway and Johnston 1965

(CA); 2. Bishop 1979 (AK)

\section{FAMILY ECHINOSTOMATIDAE Looss, 1899}

Baschkirovitrema incrassatum (Diesing, 1850)

Skrjabin, 1944

Site: stomach, small intestine

Hosts: Lutra canadensis $(1,3,4)$ Mustela vison (2)

Dist.: AL, GA, NY, NC

Records: 1. Sawyer 1961 (GA, NY); 2. Miller and Harkema 1964 (NC); 3.1968 (NC); 4.

Fleming et al. 1977 (AL)

Echinochasmus schwartzi Price, 1931

Site: small intestine

Hosts: Ondatra zibethicus $(1,2,3)$

O. z. macrodon (6)

$O$. $\boldsymbol{z}$. rivalicius $(4,5)$

Dist.: LA, MD, Ont, TX

Records: 1. Price 1931b (MD); 2. Law and Kennedy 1932 (Ont); 3. Chandler 1941 (TX); 4. Byrd and Reiber 1942b (LA); 5. Penn 1942 (LA); 6. Abram 1969 (MD)

Echinoparyphium contiguum Barker and Bastron, 1915

Site: duodenum

Host: Ondatra zibethicus $(1,2,3,4,5,6)$

O. z. obscurus (7)

Dist.: BC, KS, MI, NE, Nfld, Ont

Records: 1. Barker 1915 (NE); 2. Law and

Kennedy 1932 (Ont); 3. Ameel 1942 (MI); 4.

Knight 1951 (BC); 5. Sweatman 1952 (Ont);
6. Gash and Hanna 1972 (KS); 7. Rigby and Threlfall 1981 (Nfld)

Echinoparyphium recurvatum (vonLinstow, 1873) Luhe, 1909

Syn.: Distoma recurvatum vonLinstow, 1873

Site: small intestine

Host: Ondatra zibethicus obscurus (4)

O. z. zibethicus $(1,2,3)$

Dist.: IL, ME, Nfld, OH

Records: 1. Meyer and Reilly 1950 (ME); 2. Beckett and Gallicchio $1967(\mathrm{OH})$; 3. Jilek 1977 (IL); 4. Rigby and Threlfall 1981 (Nfld)

Echinoparyphium spp.

Site: intestine

Hosts:Alopex lagopus (3)

Ondatra zibethicus (2)

Vulpes vulpes (1)

Dist.: GD, IL, NB

Records: 1. Leigh 1940 (IL); 2. MacKinnon and Burt 1978 (NB); 3. Rausch et al. 1983 (GD)

Echinostoma callawayensis Barker and Noll, 1915 Syn.: Echinostomum callawayensis Barker and Noll, 1915

Site: duodenum

Host: Ondatra zibethicus

Dist.: AK, NE, Ont

Records: Barker 1915 (NE); Law and

Kennedy 1932 (Ont); Sweatman 1952 (Ont);

Dunagan 1957a (AK), 1957b (AK)

\section{Echinostoma echinatum}

Syn.: Distomum echinatum

Site: duodenum

Host: Ondatra zibethicus

Dist.: PA

Record: Leidy 1888

Echinostoma revolutum (Froelich, 1802) Looss, 1899

Syn.: Echinostomum armigerum Barker and Irvine, 1915

Echinostomum coalitum Barker and Beaver, 1915

Site: stomach, small intestine, cecum

Hosts: Castor canadensis (25)

Didelphis virginiana $(5,7)$

D. v. virginiana (13)

Myocastor coypus bonariensis (20)

Ondatra zibethicus

$(1,2,3,4,5,6,8,9,11,13,15,16,18,21$,

$22,27,29,31,33,35)$

O. $z$. obscurus (36)

O. $z$. occipitalis (10) 
O. z. osoyoosensis $(19,24)$

O. z. zibethicus $(12,13,14,17,23,26,32)$

Procyon lotor (29)

Ursus arctos (30)

Vulpes vulpes (34)

Dist.: BC, IL, KS, LA, MA, Man, MD, ME, MI, MN, MT, NB, NE, Nfld, NS, NY, OH, Ont, OR, PA, Que, UT, WI, WY Record: 1. Barker 1915 (NE); 2. Anonymous 1930 (Ont); 3. Law and Kennedy 1932 (Ont); 4. Krull 1935 (MD); 5. Leigh 1940 (IL); 6. Ameel 1942 (MI); 7. Byrd and Reiber 1942a (IL); 8. Rankin 1946 (MA); 9. Rausch 1946b $(\mathrm{OH}) ; 10$. Rider and Macy 1947 (OR); 11. Tiner and Chin 19 8 (IL); 12. Edwards 1949 (NY); 13. Rausch and Tiner 1949 (MI,WI); 14. Meyer and Reilly 1950 (ME); 15. Knight 1951 (BC); 16. Sweatman 1952 (Ont); 17. Gilford 1954 (IL); 18. Senger and Neiland 1955 (OR); 19. Senger and Bates 1957 (UT); 20. Babero and Lee 1961 (LA); 21. Gupta 1926b (Que); 22. Anderson and Beaudoin 1966 (PA); 23. Beckett and Gallicchio 1967 (OH); 24. Grundmann and Tsai 1967 (UT); 25. Smith and Archibald 1967 (NS); 26.

Abram 1969 (MD); 27. Gash and Hanna 1972 (KS); 28. Larson and Scharf 1975 (MN); 29. Rice and Heck $1975(\mathrm{OH}) ; 30$. Worley et al. 1976 (MT,WY); 31. Dyer and Klimstra 1977 (IL); 32. Jilek 1977 (IL); 33. MacKinnon and Burt 1978 (NB); 34. Smith 1978 (NB,NS); 35. McKenzie and Welch 1979 (Man); 36. Rigby and Threlfall 1981 (Nfld)

Echinostoma spp.

Syn.: Echinostomum sp.

Site: small intestine

Hosts: Didelphis virginiana (4)

Martes pennanti (1)

Ondatra zibethicus (2)

O. $z$. osoyooensis (3)

Dist.: Alta, CO, NC, Ont

Records: 1. Anonymous 1930 (Ont); 2.

Swales 1933 (Alta); 3. Ball 1952 (CO); 4.

Feldman et al. 1972 (NC)

Euparyphium beaveri Yamaguti, 1957

Site: duodenum, small intestine

Hosts: Mustela vison $(1,3,4)$

Procyon lotor $(2,5,6)$

Dist.: GA, LA, NC, SC, TN, WI

Records: 1. Lumsden and Zischke 1961 (LA); 2. Harkema and Miller 1964 (GA,NC,SC); 3. Miller and Harkema 1964 (NC); 4. Dorney and Lauerman 1969 (WI); 5. Bafundo et al. $1980(\mathrm{TN}) ; 6$. Schaffer et al. 1981 (NC)
Euparyphium inerme (Fuhrmann, 1904)

Syn.: Echinostomum inerme Fuhrmann, 1904

Site: intestine

Host: Mustela vison

Dist.: Ont

Records: Law and Kennedy; Price 1932

Euparyphium melis (Schrank, 1788) Dietz, 1909

Site: stomach, small intestine

Hosts:Lepus americanus phaeonotus (2)

Lutra canadensis (3)

Mustela erminea cicognanii (4)

$M$. vison $(1,3,4)$

M. v. letifera (5)

Taxidea taxus $(6,7)$

Dist.: IA, MI, MN, ND, Ont, WI

Records: 1. Law and Kennedy 1932 (Ont); 2.

Erickson 1940 (MN); 3. Beaver 1941

(MI,MN); 4. Erickson 1946 (MN); 5. Rausch

and Tiner 1949 (WI); 6. Leiby et al. 1971

(ND); 7. Wittrock and Ulmer 1974 (IA)

Euparyphium spp.

Site: small intestine

Host: Taxidea taxus

Dist.: MN

Records: Erickson 1946

Stephanoprora spinosa

Site: intestine

Host: Procyon lotor

Dist.: GA

Record: Schaffer et al. 1981

Echinostomatidae gen. sp.

Site: intestine

Host: Ondatra zibethicus

Dist.: NY

Records: Bump 1941, 1942

FAMILY FASCIOLIDAE Railliet, 1895

Fasciola hepatica Linnaeus, 1758

Site: bile ducts, liver

Hosts: Bison bison $(6,9,10)$

Bos taurus (11)

Castor canadensis (12)

Cervus elaphus (3)

Lepus americanus (12)

L. californicus merriami (5)

Odocoileus hemionus (12)

O. h. columbianus $(1,7,8)$

O. virginianus (12)

Odocoileus spp. $(3,4)$

Sus scrofa (11) 
Sylvilagus floridanus (5)

Rabbit (2)

Dist.: BC, CA, GA, LA, MT, TX, WA, WY

Records: 1. Hadwen 1916 (BC); 2. Dikmans 1930 (LA); 3. Butler 1932 (MT); 4. Herman 1945 (CA); 5. Olsen 1948 (TX); 6. Locker 1953 (MT); 7. Longhurst and Douglas 1953 (CA); 8. Browning and Lauppe 1964 (CA); 9. Bergstrom 1967 (WY); 10. Dunn 1968 (unspecified); 11. Prestwood et al. 1975 (GA); 12. Lang 1977 (WA)

Fascioloides magna (Bassi, 1875) Ward, 1917

Syn.: Distoma magnum Bassi, 1875

Site: liver

Fasciola magna (Bassi, 1875)

Hosts: Alces alces

$(28,30,31,35,40,41,51,54,57)$

A. a. americana $(5,7,44)$

Bison bison $(3,8,9,30)$

Cervus elaphus

$(9,23,24,27,28,30,33,36,41)$

C.e. nelsoni (42)

C.e. roosevelti (42)

Dicotyles tajacu angulatus (47)

Odocoileus hemionus columbianus

$(1,4,8,19,30,37,42)$

O.h. hemionus $(18,30)$

$O$. virginianus

$(1,8,9,10,11,12,13,14,15,16,17,21,25$

$26,28,29,32,34,39,42,43,44,46,50,52$,

$53,56,58,59,60$ )

O. v. borealis $(22,35)$

O. v. leucurus (61)

Odocoileus spp. $(6,38,41,48)$

Rangifer tarandus caribou $(20,49)$

Sus scrofa $(50,55)$

Dist.: AL, Alta, AR, BC, FL, GA, LA, Man, MI, MN, MS, MT, NC, NY, Ont, OR, Que, SC, TN, TX, WA, WI,

Records: 1. Stiles and Hassall 1894a (NY); 2. Hadwen 1916 (BC); 3. Cameron 1923 (Alta); 4. Hadwen 1932 (BC); 5. Fenstermacher 1934 (MN); 6. Levin 1934 (NY); 7. Wallace 1934 (MN); 8. Swales 1935 (Alta,BC), 9. 1936 (Que); 10. Bump 1937 (NY); 11. Aiton 1938 (MT); 12. Dinaburg 1939 (FL); 13. Whitlock 1939 (MI); 14. Bump 1940 (NY), 15. 1941 (NY), 16. 1942 (NY); 17. Cheatum 1942 (NY); 18. Cowan 1942 (BC), 19. 1946 (BC); 20. Erickson and Highby 1942 (MN); 21. Fenstermacher et al. $1943(\mathrm{MN}) ; 22$. Olsen and Fenstermacher 1943 (MN); 23. Kingscote 1947 (Ont); 24. Shaw 1947 (OR); 25. Chaddock 1948 (WI); 26. Olsen 1949b (TX); 27. Anonymous 1950 (Ont); 28.

Kingscote 1950 (Ont); 29. Cheatum 1951
(NY); 30. Cowan 1951 (Alta,BC); 31. Peterson 1955 (Ont); 32. Dahlberg and Guettinger 1956 (WI); 33. Kingscote 1957 (Ont); 34. Holland 1959 (AL,FL,LA, MS,SC,TN); 35. Griffiths 1962 (MN); 36. Stelfox 1962 (Alta); 37. Knapp and Shaw 1963 (OR); 38. Senger 1963 (MT); 39. Harlow and Jones 1965 (AL,FL); 40. Loken et al. 1965 (MN); 41 . Fyvie 1966 (Ont); 42. Dutson et al. 1967 (OR,WA); 43. Glazener and Knowlton 1967 (TX); 44. Dunn 1968 (unspecified); 45. Flook and Stenton 1969 (Alta,BC); 46. Samuel and Trainer 1969 (WI); 47. Samuel and Low 1970 (TX); 48. Walker and Becklund 1970 (NC); 49. Choquette et al. 1971 (Que); 50. Foreyt and Todd 1972 (TX); 51. Lankester 1972 (Man); 52. Behrend et al. 1973 (NY); 53. Foreyt and Todd 1973 (TX); 54. Lankester 1974 (Man); 55. Foreyt et al. 1975 (TX);56. Foreyt and Todd 1976 (TX); 57. Samuel et al. 1976 (Alta); 58. Foreyt et al. 1977 (TX); 59. Pursglove et al. 1977 (AL,AR,FL,GA,LA,MS,NC,SC,TN); 60. Foreyt and Drawe 1978 (TX); 61. Foreyt and Hunter 1980 (OR)

\section{FAMILY NASITREMATIDAE (Ozaki, 1953)}

Nasitrema dalli Yamaguti, 1950

Site: nasal sinus, air sinus

Host: Phocoenoides dalli

Dist.: CA

Records: Dailey 1971; Dailey and Walker 1978

Nasitrema delphini Neiland, Rice, and Holden, 1970

Site: air sinus

Host: Delphinus delphis

Dist.: CA

Record: Dailey and Walker 1978

Nasitrema globicephalae

Site: air sinus

Hosts:Delphinus delphis

Lagenorhynchus obliquidens

Lissodelphis borealis

Dist.: CA

Record: Dailey and Walker 1978

Nasitrema stenosoma Neiland, Rice, and Holden, 1970

Site: air sinus

Host: Delphinus delphis

Dist.: CA 
Record: Dailey and Walker 1978

Nasitrema spp.

Site: air sinus, brain, muscle

Hosts:Delphinus delphis $(1,2)$

Lagenorhynchus obliquidens $(1,2)$

Lissodelphis borealis $(1,2)$

Phocoenoides dalli (2)

Dist.: CA

Records: 1. Schroeder et al. 1973; 2. Dailey

and Walker 1978

\section{UNIDENTIFIED NASITREMATIDAE}

Nasitrematidae gen. spp.

Site: round window (ear)

Host: Tursiops nesarnack

Dist.: unspecified

Record: Dailey and Ridgway 1976

\section{FAMILY PSILOSTOMIDAE Looss, 1900}

Gyrosoma singularis Byrd, Bogitsh, and Maples, 1961

Site: small intestine

Hosts: Mustela vison (3) Procyon vison $(1,2)$

Dist.: GA, LA, SC

Records: 1. Byrd et al. 1961 (GA); 2.

Harkema and Miller 1964 (GA, SC); 3. Shoop

and Corkum 1982 (LA)

\section{Psilostomum ondatrae Price, 1931}

Site: liver

Host: Ondatra zibethicus

Dist.: Ont

Record: Price 1931b; Law and Kennedy 1932

\section{Psilostomum spp.}

Site: intestine

Host: Mephitis mephitis

Dist.: LA

Record: Babero 1960b

Psilotrema spp.

Site: intestine

Host: Ondatra zibethicus osoyoosensis

Dist.: UT

Record: Grundmann and Tsai 1967

Ribeiroia ondatrae Babero, 1972, nec Price, 1931

Site: small intestine

Host: Procyon lotor (2) skunks (1)

Dist.: FL
Record: 1. Babero 1972; 2. Schaffer et al. 1981

Stephanoproraoides lawi Price, 1934

Site: small intestine

Host: Castor canadensis

Dist.: MN, NS, Ont

Record: Price 1934 (Ont); Erickson 1944

(MN); Smith and Archibald 1967 (NS)

FAMILY RHOPALIASIDAE Looss, 1899

Rhopalias baculifer Braun, 1900

Site: intestine

Hosts: Didelphis marsupialis (1)

Metachirops opossum fuscogriseus (2)

Dist.: Costa Rica, FL

Records: 1. Loftin 1960 (FL); 2. Caballero

and Gei 1961 (Costa Rica)

Rhopalias coronatus (Rudolphi, 1819) Stiles and Hassall, 1898

Site: intestine

Hosts:Didelphis marsupialis (5)

D. m. caucae (4)

D. m. tabascensis $(2,3)$

D. virginiana $(1,3)$

Marmosa mexicana mexicana (3)

Metachirops opossum fuscogriseus (6)

Dist.: Costa Rica, FL, Guatemala, Mexico, TX, VA

Records: 1. Stiles and Hassall 1894a (VA); 2.

Caballero, Bravo-Hollis and Cerecoro 1944

(Mexico); 3. Caballero 1946

(Guatemala,Mexico,TX); 4. Caballero, et al. 1957 (Costa Rica); 5. Loftin 1960 (FL); 6.

Caballero and Gei 1961 (Costa Rica)

Rhopalias horridus (Diesing, 1850) Stiles and Hassall, 1898

Site: intestine

Hosts:Didelphis marsupialis caucae (2)

D. virginiana (1)

Metachirops opossum fuscogriseus (3)

Dist.: Costa Rica, Guatemala

Records: 1. Caballero 1946 (Guatemala); 2.

Caballero et al. 1957 (Costa Rica); 3.

Caballero and Gei 1961 (Costa Rica)

Rhopalias macracanthus Chandler, 1932

Site: small intestine

Hosts:Didelphis marsupialis (14)

D. m. tabascensis $(5,6)$

D. virginiana

$(1,2,3,4,7,8,10,11,12,13,15)$ 
D. v. californica (16)

Didelphis sp. (9)

Mustela vison (11)

Dist.: FL, GA, IL, LA, Mexico, NC, OK, PA, TX, TN,

Records: 1. Chandler 1932 (TX); 2. Canavan 1934 (PA); 3. Leigh 1940 (IL); 4. Byrd and

Reiber 1942a (TN); 5. Caballero, BravoHollis and Cerecoro 1944 (Mexico); 6.

Caballero 1946 (Mexico); 7. Ciordia 1949

(TN); 8. Self and McKnight 1950 (OK); 9.

Babero 1957 (IL), 10. 1960a (GA); 11.

Lumsden and Zischke 1961 (LA); 12. Miller and Harkema 1970 (NC); 13. Feldman et al. 1972 (NC); 14. Lamothe-Argumedo 1978a (Mexico), 15. Premvati and Bair 1979 (FL); 16. Miyazaki, Kifune, and LamotheArgumedo 1980 (Mexico)

\section{ORDER OPISTHORCHIIFORMES}

\section{LaRue, 1957}

FAMILY HETEROPHYIDAE Odhner, 1914

Apophallus venustus (Ransom, 1920)Cameron, 1936

Syn.: Cotylophallus venustus Ransom, 1920

Rossicotrema venustus Deliamure (1956)

Site: small intestine

Host: Alopex lagopus (1)

Phoca vitulina (4)

Procyon lotor $(2,3,4,5,7)$

Dist.: DC, GA, NC, Ont, OR, SC, VA

Records: 1. Ransom 1920 (DC); 2. Cameron 1936 (Ont), 3. 1937a (Can); 4. Babero and

Shepperson 1958 (GA); 5. Harkema and

Miller 1964 (GA, NC, SC, VA); 6. Stroud and

Dailey 1978 (OR); 7. Schaffer et al. 1981

(NC, VA)

Remarks: The Alaskan fox came from the

National Zoological Park in Washington,

D.C.

Apophallus spp.

Site: intestine

Host: Mephitis mephitis

Dist.: Que

Record: Webster and Wolfgang 1956

Remarks: Webster and Wolfgang (1956)

believed their species may be Apophallus

venustus.

Ascocotyle ampullacea Miller and Harkema, 1962

Site: small intestine

Host: Procyon lotor
Dist.: NC

Records: Miller and Harkema 1962;

Harkema and Miller 1964

Ascocotyle leighi Burton, 1956

Site: small intestine

Hosts:Procyon lotor (2)

Dist.: SC

P. l. solutus (1)

Records: 1. Harkema and Miller 1962, 2.

1964

Ascocotyle pachycystis Schroeder and Leigh, 1965

Site: small intestine

Host: Procyon lotor

Dist.: FL

Record: Schroeder and Leigh 1965

Cotylophallus similis Ransom, 1920

Site: intestine

Host: Phoca vitulina

Dist.: DC

Record: Ransom 1920

Remarks: The Harbor seal came from the

National Zoological Park in Washington,

D.C.

Cryptocotyle concavum (Creplin, 1825)

Site: intestine

Host: Alopex lagopus

Dist.: GD

Record: Rausch et al. 1983

Cryptocotyle jejuna (Nicoll, 1907)

Site: small intestine

Host: Callorhinus ursinus

Dist.: AK

Record: Neiland 1961

Cryptocotyle lingua (Creplin, 1825) Fischoeder, 1903

Syn.: Distomum lingua Creplin, 1825

Site: intestine

Hosts:Phoca vitulina (1)

Vulpes vulpes (2)

Dist.: DC, NB, NS

Record: 1. Ransom 1920 (DC); 2. Smith 1978 (NB,NS)

Remarks: The specimens were collected by

Dr. Albert Hassall from the intestine of

Phoca vitulina housed at the National

Zoological Park, Washington, D.C.

Euryhelmis monorchis Ameel, 1938

Site: small intestine

Hosts: Mustela vison (1)

Procyon lotor lotor (2) 
Dist.: MI, OH, WI

Records: 1. Ameel 1938 (MI,OH,WI); 2.

Rausch and Tiner 1949 (MI)

Euryhelmis pacificus Senger and Macy, 1952

Site: small intestine

Hosts:Mustela vison $(1,2)$

Ondatra zibethicus $(1,2)$

Sorex bendirii palmeri (1)

Dist.: OR

Records: 1. Senger and Macy 1952; 2. Senger and Neiland 1955

Euryhelmis pyriformis Webster and Wolfgang, 1956

Site: intestine

Host: Mephitis mephitis

Dist.: Que

Record: Webster and Wolfgang 1956

Euryhelmis squamula (Rudolphi, 1819)

Site: small intestine

Hosts: Mustela vison $(2,5)$

Peromyscus leucopus (7)

Procyon lotor $(1,3,4,8)$

Urocyon cinereoargenteus (6)

Dist.: GA, NC, OR, TN, WV

Records: 1. Parker 1950 (NC); 2. Senger and

Neiland 1955 (OR); 3. Babero and

Shepperson 1958 (GA); 4. Harkema and

Miller 1964 (NC); 5. Miller and Harkema 1964 (NC), 6. 1968 (GA); 7. Zenchak and

Hall 1971 (WV); 8. Bafundo et al. 1980 (TN)

Metagonimoides oregonensis Price, 1931

Site: small intestine

Hosts: Mustela vison $(4,7,11)$

Procyon lotor $(1,2,5,6,9,10,12,13)$

P. l. lotor (8)

$P$. l. pacificus (3)

Dist.: GA, MI, NC, OH, OR, TN, VA

Records: 1. Price 1931a (OR); 2. Shaw 1947

(OR); 3. Burns and Pratt 1953 (OR); 4.

Senger and Neiland 1955 (OR);5. Sawyer

1958 (GA); 6. Harkema and Miller 1964

(NC); 7. Miller and Harkema 1964 (NC); 8.

Williams and Myer $1964(\mathrm{OH}) ; 9$. Wallace and Wilson 1965 (TN); 10. Lang et al. 1968

(NC); 11. Lang and Vail 1970 (MI); 12.

Bafundo et al. 1980 (TN); 13. Schaffer 1981

(GA,NC,VA)

Phagicola diminuta (Stunkard and Haviland, 1924)

Syn.: Ascocotyle diminuta Stunkard and Haviland, 1924
Parascocotyle lageniformis (Chandler, 1941)

Phagicola lageniformis Chandler, 1941

Site: small intestine

Hosts:Didelphis virginiana (7)

Ondatra zibethicus (2)

Procyon lotor $(5,6)$

P. l. solutus (4)

Rattus sp. $(1,3)$

Dist.: FL, NC, NY, SC, TX

Records: 1. Stunkard and Haviland 1924

(NY); 2. Chandler 1941 (TX); 3. Stunkard and Uzmann 1955 (NY); 4. Harkema and Miller 1962 (SC); 5. Miller and Harkema 1963 (NC,SC); 6. Harkema and Miller 1964 (NC,SC); 7. Premvati and Bair 1979 (FL)

Phagicola longa (Ransom, 1920) Price, 1935

Syn.: Ascocotyle longa Ransom, 1920

Site: small intestine

Hosts:Alopex lagopus (1)

Procyon lotor $(4,5)$

P. l. solutus (3)

Vulpes vulpes (2)

Dist.: DC, NC, Ont, Que, SC

Records: 1. Ransom 1920 (DC); 2. Swales

1933 (Ont,Que); 3. Harkema and Miller 1962

(SC); 4. Miller and Harkema 1963 (NC,SC);

5. Harkema and Miller 1964 (NC,SC)

Remarks: Ransom's material was described from an Alaskan fox housed at the National Zoological Park, Washington, D.C.

Phagicola nana (Ransom, 1920)

Syn.: Ascocotyle nana Ransom, 1920

Site: small intestine

Host: Alopex lagopus (1)

Ondatra zibethicus rivalicius (2)

Dist.: DC, LA

Records: 1. Ransom 1920 (DC); 2. Byrd and

Reiber 1942b (LA)

Remarks: Ransom's (1920) material was

from an Alaskan fox housed at the National

Zoological Park, Washington, D.C.

Phocitrema fusiforme Goto and Ozaki,1930

Site: small intestine

Hosts:Callorhinus ursinus (3)

Enhydra lutris $(1,2)$

Phoca vitulina largha (4)

Dist.: AK

Records: 1. Rausch and Locker 1951; 2.

Rausch 1953; 3. Neiland 1961; 4. Shults 1982

Pholeter gastrophilus (Kossack, 1910) 
Syn.: Distomum gastrophilum Kossack, 1910

Site: stomach

Hosts:Steno bredanensis (3)

Tursiops gillii (2)

T. nesarnack $(1,2)$

Dist.: FL

Records: 1. Woodard et al. 1969 (FL); 2.

Migaki et al. 1971 (unspecified); 3. Forrester and Robertson 1975 (FL)

Pricetrema eumetopii Shults, 1978

Site: small intestine

Host: Eumetopias jubatus

Dist.: AK

Records: Shults 1978

Pricetrema phocae Shults, 1978

Site: intestine

Host: Phoca vitulina richardsii

Dist.: AK

Records: Shults 1978

Pricetrema zalophi (Price, 1932)

Syn.: Apophallus zalophi Price, 1932

Site: small intestine

Host: Callorhinus ursinus (3)

Enhydra lutris $(1,2)$

Eumetopias jubatus (4)

Mirounga angustirostris (4)

Phoca vitulina (4)

Zalophus californianus (4)

Dist.: AK, OR

Records: 1. Rausch and Locker 1951 (AK); 2.

Rausch 1953 (AK); 3. Neiland 1961 (AK); 4.

Stroud and Dailey 1978 (OR)

Pricetrema spp.

Site: small intestine

Host: Erignathus barbatus

Dist.: AK

Record: Bishop 1979

\section{FAMILY OPISTHORCHIIDAE Looss, 1899}

Amphimerus neotropicalis Caballero, Gei, and Caballero, 1963

Site: pancreatic duct

Host: Metachirops opossum fuscogriseus

Dist.: Costa Rica

Record: Caballero, Gei and Caballero 1963

Amphimerus pseudofelineus (Ward, 1901) Barker, 1911

Site: bile duct, gall bladder

Host: Didelphis virginiana (1)
Ondatra zibethicus zibethicus (2)

Dist.: IL

Record: 1. Leigh 1940; 2. Gilford 1954

Metorchis conjunctus (Cobbald, 1860) Looss, 1899

Site: bile ducts, gall bladder, pancreas

Hosts: Canis latrans (3)

Canis lupus $(3,8)$

Martes pennanti (5)

Ondatra zibethicus (2)

Procyon lotor (6)

Sigmodon hispidus (7)

Vulpes vulpes (4)

Dist.: Alta, Man, NB, NS, Ont, PA, Que, Sask, VA

Records: 1. Sweatman 1952 (Ont); 2.

Anderson and Beaudoin 1966 (PA); 3.

Holmes and Podesta 1968 (Alta); 4. Smith

1978 (NB,NS); 5. Dick and Leonard 1979

(Man); 6. Schaffer et al. 1981 (VA); 7.

Watson 1981 exp. (Que); 8. Wobeser et al. 1983 (Sask)

Opisthorchis pricei Foster, 1939

Site: bile ducts

Host: Caluromys derbianus pallidus

Dist.: Panama

Record: Foster 1939

Opisthorchis tenuicollis (Rud. 1819)

Site: bile duct

Host: Lynx canadensis

Dist.: $\mathrm{Pa}$

Record: Canavan 1934

Remarks: The specimens came from an

animal from the Pennsylvania Zoo.

Opisthorchis tonkae Wallace and Penner, 1939

Site: bile duct, gall bladder, pancreas, small

intestine

Hosts: Ondatra zibethicus $(1,3)$

O. z. zibethicus (2)

Dist.: MN, NB, NY

Records: 1. Wallace and Penner 1939 (MN);

2. Edwards 1949 (NY); 3. MacKinnon and

Burt 1978 (NB)

Parametorchis canadensis Price, 1929

Site: gallbladder

Host: Mustela vison

Dist.: Ont

Records: Price 1929; Anonymous 1930, 1931; Law and Kennedy 1932

Parametorchis complexus (Stiles and Hassall, 1894)

Site: liver 
Host: Procyon lotor

Dist.: NC, VA

Records: Harkema and Miller 1964 (NC);

Schaffer et al. 1981 (NC,VA)

Parametorchis spp.

Site: liver

Host: Ondatra zibethicus

Dist.: MD

Record: Smith 1938

\section{ORDER PARAMPHISTOMIFORMES}

\section{Szidat, 1936}

FAMILY NOTOCOTYLIDAE Luhe, 1909

Catatropis fimbriata Barker, 1915

Site: duodenum

Host: Ondatra zibethicus

Dist.: NE

Record: Barker 1915

Catatropis johnstoni Martin, 1956

Site: cecum and large intestine

Host: Oryzomys palustris

Dist.: FL

Record: Bush and Kinsella 1972

Notocotylus filamentis (Barker, 1915) Harwood, 1939

Syn.: Catatropis filamentis Barker, 1915

Site: large intestine

Hosts:Ondatra zibethicus $(1,2,3,4,6,7,8,9)$

O. z. zibethicus (5)

Dist.: AK, IL, ME, Man, MI, Ont

Records: 1. Law and Kennedy 1932 (Ont); 2.

Harwood 1939 (MI); 3. Leigh 1940 (IL); 4.

Ameel 1942 (MI); 5. Meyer and Reilly 1950

(ME); 6. Sweatman 1952 (Ont); 7. Dunagan

1957a (AK), 8. 1957b (AK); 9. McKenzie and

Welch 1979 (Man)

Notocotylus hassalli McIntosh and McIntosh, 1934

Site: cecum, small intestine

Hosts:Microtus pennsylvanicus

pennsylvanicus $(1,2)$

Zapus hudsonius hudsonius (3)

Dist.: MD, MI, VA

Records: 1. Stiles and Hassall 1894a (MD); 2.

McIntosh and McIntosh 1934 (MD,VA); 3.

Erickson 1938 (MI)

Remarks: Stiles and Hassall recorded their trematode as Monostoma sp.. McIntosh and McIntosh reexamined the Monostoma sp. and believed it to be $N$. hassalli.
Notocotylus urbanensis (Cort, 1914) Harrah, 1922

Site: large intestine

Hosts: Ondatra zibethicus $(3,4,7,8)$

$$
\begin{aligned}
& \text { O.z.occipitalis (1) } \\
& \text { O.z. osoyoosensis }(6) \\
& \text { O.z.zibethicus }(2,5)
\end{aligned}
$$

Dist.: BC, MI, NB, NY, OH, Ont, OR, PA, UT Records: 1. Rider and Macy 1947 (OR); 2.

Edwards 1949 (NY); 3. Knight 1951 (BC); 4. Herber 1955 (MI,PA); 5. Beckett and Gallicchio $1967(\mathrm{OH})$; 6. Grundmann and Tsai 1967 (UT); 7. Beverley-Burton and Sweeny 1972 (Ont); 8. MacKinnon and Burt $1978(\mathrm{NB})$

Notocotylus spp.

Site: intestine

Hosts:Ondatra zibethicus (2)

O. z. zibethicus (1)

Dist.: IL, OR

Records: 1. Gilford 1954 (IL); 2. Senger and Neiland 1955 (OR)

Nudacotyle novicia Barker, 1916

Site: bile duct, stomach, small intestine, large intestine

Hosts:Microtus pennsylvanicus pennsylvanicus (6) Ondatra zibethicus $(1,2,3,4,6,13)$

O. z. macrodon (10)

O. $z$. rivalicius $(5)$

O. $z$. zibethicus $(7,8,9)$

Sciurus carolinensis (12) Sylvilagus palustris (11)

Dist.: AL, IA, IL, LA, MD, ME, MI, MN, NB, $\mathrm{NC}, \mathrm{OH}$, Ont, TX

Records: 1. Barker $1916(\mathrm{MN})$; 2. Law and Kennedy 1932 (Ont); 3. Chandler 1941 (TX); 4. Ameel 1942 (MI); 5. Penn 1942 (LA); 6. Ameel 1944 (IA,MI); 7. Meyer and Reilly 1950 (ME); 8. Gilford 1954 (IL); 9. Beckett and Gallicchio $1967(\mathrm{OH}) ; 10$. Abram 1969 (MD); 11. Stringer et al. 1969 (NC); 12. Davidson 1976 (AL); 13. MacKinnon and Burt 1978 (NB)

Nudacotyle spp.

Site: small intestine

Host: Sciurus carolinensis carolinesis

Dist.: TN

Record: Olexik et al. 1969

Ogmogaster antarctica Johnston, 1931

Syn.: Ogmogaster plicatus of Margolis and Pike (1955), in part

Site: rectum, large intestine

Hosts:Balaenoptera borealis (1) 
B. physalus (2)

Eschrichtius gibbosus (3)

Dist.: CA, BC

Records: 1. Rausch and Fay 1966 (BC); 2.

Rausch and Rice 1970 (CA); 3. Rice and

Wolman 1971 (CA)

Ogmogaster pentalineatus Rausch and Fay, 1966

Site: small intestine, rectum

Host: Eschrichtius gibbosus

Dist.: AL, CA

Records: Rausch and Fay 1966 (AL,CA);

Rice and Wolman 1971 (CA)

Ogmogaster plicatus (Creplin, 1829) Jagerskiold, 1891

Site: rectum, small intestine

Hosts:Balaenoptera borealis (1)

B. physalus $(1,2,3,4)$

Balaenoptera sp. (3)

Dist.: CA, BC

Records: 1. Margolis and Pike 1955 (BC); 2.

Rausch and Fay 1966 (BC); 3. Rausch and

Rice 1970 (CA); 4. Rice and Wolman 1971

(CA); 5. Gibson and Harris 1979 (BC)

Ogmogaster trilineatus Rausch and Rice, 1970

Site: rectum

Host: Balaenoptera physalus

Dist.: CA

Record: Rausch and Rice 1970

Paramonostomum pseudalveatum Price, 1931

Site: small intestine, large intestine

Hosts:Ondatra zibethicus (1)

O. z. osoyoosensis (3)

O. $z$. rivalicius $(2)$

Dist.: LA, MD, UT

Records: 1. Price 1931b (MD); 2. Penn 1942

(LA); 3. Grundmann and Tsai 1967 (UT)

Quinqueserialis floridensis Rausch, 1952

Site: cecum

Host: Neofiber alleni nigrescens

Dist.: FL

Record: Rausch 1952a

Quinqueserialis hassalli (McIntosh and McIntosh, 1934) Harwood, 1939

Site: cecum

Hosts:Marmota monax (2)

Microtus montanus fusus (5)

M. m. nanus $(4,5)$

$M$. pennsylvanicus modestus $(4,5)$

Thomomys talpoides (5)

Dist.: CO, MD, MN, NM, VA, WI, WY
Records: 1. Harwood 1939 (MD,MN,VA); 2.

Rausch and Tiner 1948 (WI); 3. 1949 (WI); 4.

Kuns and Rausch 1950 (WY); 5. Smith 1954

(CO,NM,WY)

Quinqueserialis quinqueserialis (Barker and Laughlin, 1911) Harwood, 1939

Syn.: Notocotyle quinqueseriale Barker and Laughlin, 1911

Site: small intestine, large intestine, cecum

Hosts: Microtus oeconomus macfarlani (14)

M. pennsylvanicus (17)

M.p. drummondii (14)

Ondatra zibethicus $(1,2,3,4,5,6,7,11$,

$12,14,15,18,19,20,22$,

$23,27,28,29,30,31,33,34)$

O. z. obscurus (35)

O.z. osoyoosensis $(13,21,25)$

O. z. zibethicus $(8,9,10,16,24,26,32)$

Zapus hudsonius (17)

Dist.: AK, BC, CO, IA, IL, Lab, Man, MD, ME, MI, MT, NB, NE, Nfld, NY, OH, Ont, OR, PA, Que, UT, WI

Records: 1. Barker and Laughlin 1911 (NE); 2. Barker 1915 (NE); 3. Law and Kennedy 1932 (Ont); 4. Harwood 1939 (IA,MD,MT);5. Ameel 1942 (MI); 6. Herber 1942 (MI,PA); 7. Rausch 1946b (OH); 8. Edwards 1949 (NY); 9. Rausch and Tiner 1949 (WI); 10. Meyer and Reilly 1950 (ME); 11. Dubois 1951 (MD); 12. Knight 1951 (BC); 13. Ball 1952 (CO); 14. Rausch 1952b (AK); 15. Sweatman 1952 (Ont); 16. Gilford 1954 (IL); 17. Schad 1954 (Nfld,Que); 18. Senger and Neiland 1955 (OR); 19. Dunagan 1957a (AK), 20. 1957b (AK); 21. Senger and Bates 1957 (UT); 22. Arata 1959 (IL); 23. Anderson and Beaudoin 1966 (PA); 24. Beckett and Gallicchio 1967 (OH); 25. Grundmann and Tsai 1967 (UT); 26. Abram 1969 (MD); 27. Kinsella 1971 (MT); 28. Beverley-Burton and Sweeny 1972 (Ont); 29. Rice and Heck 1975 (OH); 30. Wittrock 1976 (IA); 31. Dyer and Klimstra 1977 (IL); 32. Jilek 1977 (IL); 33.

MacKinnon and Burt 1978 (NB); 34.

McKenzie and Welch 1979 (Man); 35. Rigby and Threlfall 1981 (Nfld)

Quinqueserialis zibethicai Gupta 1962

Site: duodenum

Host: Ondatra zibethicus

Dist.: Que

Record: Gupta 1962a (Que)

Quinqueserialis spp.

Site: intestine

Host: Marmota monax 
Dist.: WI

Record: Rausch and Tiner 1949

Notocotylidae of Undetermined Species

Monostomum affinis Leidy, 1858

Site: gall bladder and bile duct

Host: Ondatra zibethicus

Dist.: PA

Record: Leidy 1858

\section{FAMILY PARAMPHISTOMIDAE}

Fischoeder, 1901

Calicophoron microbothrioides (Price and

McIntosh, 1944) Eduardo, 1983

Syn.: Paramphistomum microbothrioides

Price and McIntosh, 1944

Site: rumen

Host: Bos taurus

Dist.: GA

Record: Prestwood et al. 1975

Chiorchis fabaceus (Diesing, 1838)

Syn.: Amphistoma fabaceum Diesing, 1838

Site: nasal passages, intestine, colon

Host: Trichechus manatus

Dist.: FL, PA

Record: Leidy 1890 (PA); Stiles and Hassall 1894a (FL); Canavan 1934 (PA)

Remarks: Liedy (1890) collected specimens from a seacow that died in the Zoological Gardens in 1875. Canavan (1934) obtained specimens from a manatee that died at the Pennsylvania Zoo.

Paramphistomum cervi (Zeder, 1790) Fischoeder, 1901

Site: rumen

Hosts: Alces alces $(1,2,4,5,7,9,10)$
A. a. americana $(3,8)$
Odocoileus virginianus $(6,8)$

Dist.: AL, MN, Nfld, Ont, Que, Sask

Records: 1. Fenstermacher and Jellison 1933

(MN); 2. Fenstermacher $1934(\mathrm{MN}) ; 3$.

Wallace 1934 (MN); 4. Fenstermacher 1937

(MN); 5. Peterson 1955 (Ont,Que); 6. Harlow and Jones 1965 (AL); 7. Threlfall 1967

(Nfld); 8. Dunn 1968 (unspecified); 9.

Lankester et al. 1979 (Ont); 10. Kennedy et al. 1985 (Ont,Sask)

Paramphistomum liorchis Fischoeder, 1901

Site: rumen, small intestine

Hosts: Alces alces (5)
Cervus unicolor (6)

Odocoileus virginianus $(1,2,6)$

Odocoileus spp. $(3,4)$

Dist.: AL, FL, GA, LA, Ont, SC

Records: 1. Dunn 1968 (unspecified); 2.

Prestwood et al. 1970 (AL,FL,GA,LA,SC); 3.

Prestwood et al. 1975 (GA); 4. Walker and

Becklund 1970 (AL); 5. Kennedy et al. 1985

(Ont); 6. Davidson et al. 1987 (FL)

Paramphistomum spp.

Site: rumen

Host: Alces alces

Dist.: Alta, MN

Record: Loken et al. 1965 (MN); Samuel et al. 1976 (Alta)

Stichorchis subtriquetrus (Rudolphi, 1814)

Fischoeder, 1901

Syn.: Amphistomum subtriquetrum

Rudolphi, 1819

Paramphistomum castori Kofoid and Park, 1937

Site: intestine, cecum

Hosts: Castor canadensis

$(2,3,6,8,9,10,11,12,13,14,15,16)$

C. c. carolinensis (4)

C. c. leucodontus (5)

C. c. mexicanus (7)

Ondatra zibethicus (1)

Dist.: AK, Alta, BC, CO, LA, MEXICO, MN, Nfld, NS, Ont, OR, PA, Que, TX

Records: 1. Leidy 1888 (PA); 2. Duff 1914

(Ont,Que); 3. Law and Kennedy 1932 (Ont);

4. Bennett and Humes 1939 (LA); 5. Cowan

1942 (BC); 6. Erickson 1944 (MN); 7.

Caballero 1947 (Mexico); 8. Shaw 1947 (OR);

9. Olsen 1949a (CO); 10. Babero 1953 (AK);

11. Senger and Neiland 1955 (OR); 12.

Choquette and Pimlott 1956 (Nfld); 13.

Dunagan 1957a (AK); 14. Smith and

Archibald 1967 (NS); 15. Fedynich et al.

1986 (TX); 16. Bush and Samuel 1981 (Alta)

Wardius zibethicus Barker and East, 1915

Syn.: Pseudodiscus zibethicus (Barker and East, 1915) Fukui, 1929

Site: cecum

Hosts:Ondatra zibethicus
$(1,2,3,4,8,9,10,13,14,15)$
O. $z$. zibethicus $(4,5,6,7,11,12)$

Dist.: IL, Man, MD, ME, MI, NB, NE, NY, $\mathrm{OH}$, Ont, PA

Records: 1. Barker 1915 (NE); 2. Law and

Kennedy 1932 (Ont); 3. Ameel 1942 (MI); 4.

Rausch 1946b (OH); 5. Edwards 1949 (NY);

6. Meyer and Reilly 1950 (ME); 7. Gilford 
1954 (IL); 8. Smith 1958 (MI); 9. Murrell 1965 (MI); 10. Anderson and Beaudoin 1966 (PA); 11. Beckett and Gallicchio $1967(\mathrm{OH})$; 12. Abram 1969 (MD); 13. Rice and Heck $1975(\mathrm{OH}) ; 14$. MacKinnon and Burt 1978 (NB); 15. McKenzie and Welch 1979 (Man)

Zygocotyle lunata (Diesing, 1836)

Site: cecum

Hosts: Alces alces $(2,3)$

Odocoileus virginianus (1)

Dist.: Alta, MI

Record: 1. Swanson 1960 (MI); 2. Samuel et al. 1976 (Alta); 3. Stock and Barrett 1983 (Alta)

\section{UNIDENTIFIED PARAMPHISTOMIDAE}

Paramphistomidae gen. spp.

Site: rumen

Hosts: Odocoileus virginianus (1)

Ondatra zibethicus obscurus (2)

Dist.: FL, Nfld

Record: 1. Dinaburg 1939 (FL); 2. Rigby and

Threlfall 1981 (Nfld)

\section{ORDER PLAGIORCHIIFORMES}

\section{LaRue, 1957}

FAMILY ALLASSOGONOPORIDAE Odening, 1964

Allassogonoporus marginalis Olivier, 1938

Site: small intestine

Hosts:Eptesicus fuscus (8)

Myotis grisescens $(3,4)$

M. lucifugus $(5,7)$

M. sodalis (2)

$M$. velifer incautus (3)

Ondatra zibethicus (1)

Tadarida brasiliensis cynocephala (6)

Dist.: IA, KS, KY, LA, MI, WI

Records: 1. Olivier 1938 (MI); 2. Williams 1962 (KY); 3. Ubelaker 1966 (KS); 4. Nickel and Hansen 1967 (KS); 5. Blankespoor and Ulmer 1970 (IA); 6. Martin 1976 (LA); 7.

Coggins et al. 1982 (WI); 8. Lotz and Font 1983 (WI)

Allassogonoporus vespertilionis Macy, 1940

Site: intestine

Hosts: Eptesicus fuscus (3)

Myotis californicus caurinus $(1,2)$

M. lucifugus (3)

Dist.: OR
Records: 1. Macy 1940c, 2. 1947; 3. Knight and Pratt 1955

Cephalophallus obscurus Macy and Moore, 1954

Site: intestine

Host: Mustela vison

Dist.: MI, OR

Record: 1. Macy and Moore 1954 (OR); 2.

Lang and Dronen 1972 (MI)

Myotitrema asymmetrica Macy, 1940

Site: intestine

Host: Myotis lucifugus

Dist.: MN

Record: Macy 1940a

\section{FAMILY ANENTEROTREMATIDAE}

Anenterotrema freitasi Caballero, 1964

Site: intestine

Host: Micronycteris hirsuta

Dist.: Costa Rica

Record: Caballero 1964b

FAMILY DICROCOELIIDAE Looss, 1899

Brachylecithum spp.

Site: bile ducts

Host: Blarina brevicauda

Dist.: NC

Record: Miller et al. 1974

Conspicuum icteridorum Denton and Byrd, 1951

Syn.: Quiscalus spp. Denton and Byrd, 1951

Site: bile ducts

Hosts:Tadarida brasiliensis cynocephala T. b. mexicana

Dist.: LA, TX

Record: Martin 1976

Dicrocoelium dendriticum (Rudolphi, 1819) Looss, 1899

Site: bile duct, liver

Hosts:Lepus americanus struthopus (4)

Marmota monax rufescens (2)

Mustela spp. (1)

Odocoileus virginianus (3)

Dist.: Nfld, NY, PEI

Records: 1. Conklin and Baker 1930 (PEI); 2. Mapes 1950 (NY); 3. Mapes and Baker 1950

(NY); 4. Dodds and Mackiewicz 1961 (Nfld);

5. Dunn 1968 (unspecified)

Dicrocoelium lasiuri McIntosh, 1933

Site: bile ducts, gall bladder 
Host: Nycteris borealis

Dist.: DC

Records: 1. McIntosh 1933; 2. McIntosh and McIntosh 1935

Dicrocoelium rileyi Macy, 1932

Site: bile ducts, gall bladder

Hosts: Eptesicus fuscus (7) Myotis velifer incautus (6)

Nycticeius humeralis (2)

Tadarida brasiliensis (4)

T. b. cynocephala $(1,3,8)$

T. b. mexicana $(5,6,8)$

Dist.: IA, KS, LA, NM, OK, TX

Records: 1. Macy 1932 (KS,OK); 2. Chandler 1938 (TX); 3. Byrd and Macy 1942 (LA); 4. Jameson 1959 (TX); 5. Cain 1966 (NM); 6. Nickel and Hansen 1967 (KS,OK); 7.

Blankespoor and Ulmer 1970 (IA); 8. Martin 1976 (LA,TX)

Dictyonograptus chamelensis Lamothe-Argumedo, 1980

Site: bile ducts

Host: Peromyscus banderanus banderanus

Dist.: Mexico

Record: Lamonthe-Argumedo 1980

Eurytrema allentoshi (Foster, 1939)

Syn.: Platynosomum allentoshi Foster, 1939

Site: bile ducts, gall bladder

Host: Caluromys derbianus pallidus (1)

Didelphis virginiana (2)

Dist.: Panama, TX

Record: 1. Foster 1939 (Panama); 2. Denton 1944a (TX)

Eurytrema komareki McIntosh, 1939

Site: stomach

Host: Peromyscus gossypinus gossypinus

Dist.: GA

Record: McIntosh 1939

Eurytrema procyonis Denton, 1942

Syn.: Eurytrema vulpis Stunkard, 1947

Site: pancreatic ducts, small intestine

Hosts: Canis latrans $x$ Canis rufus (12)

Procyon lotor $(2,3,4,5,6,7,8,9,11,12,13)$

P. l. lotor (1)

Urocyon cinereoargenteus $(6,10)$

Vulpes vulpes (4)

Fox (14)

Dist.: CT, GA, LA, MD, NC, NY, TN, TX, VA

Records: 1. Chandler 1942a (TX); 2. Denton 1942 (TX), 3. 1944b (TX); 4. Stunkard 1947

(NY); 5. Penner et al. 1954 (CT); 6. Herman et al. 1957 (MD); 7. Babero and Shepperson
1958 (GA); 8. Sawyer 1958 (GA); 9.

Harkema and Miller 1964 (NC,VA); 10.

Miller and Harkema 1968 (NC); 11. Bafundo et al. 1980 (TN); 12. Custer and Pence 1981

(TX); 13. Schaffer et al. 1981 (VA); 14. Foley et al. 1987 (NY)

Lyperosomum intermedium Denton and Kinsella, 1972

Site: pancreas

Host: Oryzomys palustris

Dist.: FL, GA

Record: Denton and Kinsella 1972

Lyperosomum sinuosum Travassos, 1917

Site: pancreatic ducts, small intestine

Hosts:Procyon lotor $(2,3)$

P. l. solutus (1)

Dist.: SC, TE

Records: 1. Harkema and Miller 1962 (SC), 2.

1964 (SC); 3. Bafundo et al. 1980 (TE)

Platynosomum beltrani Caballero and Caballero, 1969

Site: intestine

Host: Tadarida brasiliensis mexicana

Dist.: Mexico

Record: Caballero and Caballero 1969b

Robertdigenea dollfusi Caballero and Caballero, 1969

Site: bile ducts and gall bladder

Host: Procyon lotor crassidens

Dist.: Costa Rica

Record: Caballero and Caballero 1969a

Zonorchis komareki (McIntosh, 1939) Travassos, 1944

Site: bile ducts

Hosts:Oryzomys palustris (1)

Reithrodontomys humulis (2)

Dist.: GA, LA

Records: 1. Lumsden and Zischke 1961 (LA);

2. McKeever 1971 (GA)

\section{FAMILY LECITHODENDRIIDAE Odhner, 1910}

Acanthatrium alicatai Macy, 1940

Site: intestine

Host: Eptesicus fuscus

Dist.: DC

Record: Macy 1940b

Acanthatrium amphidymum Cheng, 1957

Site: small intestine 
Host: Eptesicus fuscus fuscus

Dist.: VA

Record: Cheng 1957, 1959

Acanthatrium beuschleini Cheng, 1959

Site: small intestine

Hosts: Eptesicus fuscus fuscus Myotis lucifugus

Dist.: VA

Record: Cheng 1959

Acanthatrium eptesici Alicata, 1932

Site: small intestine

Hosts: Eptesicus fuscus $(1,2,5,6)$

E. f. fuscus (3)

Nycteris borealis (4)

Dist.: BC, DC, IA, MN, VA, WI

Records: 1. Alicata 1932 (DC); 2. Macy

1940b (MN); 3. Cheng 1959 (VA); 4.

Blankespoor and Ulmer 1970 (IA); 5.

Webster and Casey 1973 (BC); 6. Lotz and

Font 1983 (WI)

Acanthatrium microcanthum Macy, 1940

Site: small intestine

Host: Eptesicus fuscus

Dist.: MN, WI

Records: Macy 1940b(MN); Lotz and Font 1983 (WI)

Acanthatrium nycteridis Faust, 1919

Site: small intestine

Hosts: Eptesicus fuscus fuscus (3)

Lasionycteris noctivagans (4)

Myotis lucifugus lucifugus (2)

Nycteris borealis borealis (1)

Tadarida brasiliensis mexicana (5)

Dist.: BC, IL, MA, TX, VA

Records: 1. Faust 1919 (IL); 2. Rankin 1946

(MA); 3. Cheng 1959 (VA); 4. Webster and

Casey 1973 (BC); 5. Martin 1976 (TX)

Acanthatrium oligacanthum Cheng, 1957

Site: small intestine

Hosts: Eptesicus fuscus (2)

E.f. fuscus (1)

Dist.: VA, WI

Records: 1. Cheng 1957 (VA); 2. Lotz and

Font 1983 (WI)

Acanthatrium oregonense Macy, 1939

Site: intestine

Hosts: Myotis californicus caurinus $(1,2)$

$M$. evotis evotis $(1,2)$

M. lucifugus (3)

Dist.: OR
Records: 1. Macy 1939 , 2. 1947; 3. Knight and Pratt 1955

Acanthatrium pipistrelli Macy, 1940

Syn.: Acanthatrium lunatum Williams, 1960

Site: small intestine

Hosts:Eptesicus fuscus $(3,4)$

E.f. fuscus (2)

Myotis sodalis (2)

Nycteris borealis (3)

Pipistrellus subflavus (1)

Dist.: IA, KY, MN, OH, WI

Records: 1. Macy 1940b (MN); 2. Williams

1962 (KY); 3. Blankespoor and Ulmer 1970

(IA); 4. Lotz and Font 1983 (MN,WI)

Acanthatrium umbraculatum Christian, 1972

Site: small intestine

Host: Myotis lucifugus

Dist.: $\mathrm{OH}$

Record: Christian 1972

Acanthatrium spp.

Site: intestine

Host: Myotis grisescens

Dist.: KS

Record: Nickel and Hansen 1967

Castroia silvai Travassos, 1928

Site: intestine

Host: Micronycteris hirsuta

Dist.: Costa Rica

Record: Caballero and Gei 1963

Glyptoporus noctophilus Macy, 1936

Site: small intestine

Hosts: Eptesicus fuscus (2)

Dist.: MN

Myotis lucifugus (1)

Records: 1. Macy 1936a; 2. Lotz and Font 1983

Gyrabascus brevigastrus Macy, 1935

Site: small intestine

Host: Myotis lucifugus

Dist.: WI

Record: Macy 1935a

Lecithodendrium anticum Stafford, 1905

Site: intestine

Host: Myotis lucifugus

Dist.: Not specified

Record: Stafford 1905

Lecithodendrium posticum Stafford, 1905

Site: intestine

Host: Myotis lucifugus 
Dist.: Not specified

Record: Stafford 1905

Lecithodendrium spp.

Site: small intestine

Hosts: Eptesicus fuscus fuscus (1) Nycteris intermedia floridana (2)

Dist.: FL, VA

Records: 1. Cheng 1959 (VA); 2. Loftin 1960

(FL)

Limatulum costarricensis (Caballero y Brenes, 1957) Caballero, 1964

Site: intestine

Host: Myotis nigricans nigricans

Dist.: Panama

Records: Caballero 1964a

Limatulum gastroides Macy, 1935

Site: stomach, intestine

Hosts: Myotis californicus caurinus (3)

M. keenii (7)

$M$. lucifugus $(1,5,8)$

M. l. lucifugus (2)

M. sodalis (4)

Nycteris cinerea (6)

Dist.: BC, IA, KY, MA, MN, OR, WI

Records: 1. Macy 1935b (MN,WI); 2. Rankin 1946 (MA); 3. Macy 1947 (OR); 4. Williams 1962 (KY); 5. Blankespoor and Ulmer 1970 (IA); 6. Webster and Casey 1973 (BC); 7. Coggins et al. 1981 (WI), 8.1982 (WI)

Limatulum istmicus Caballero 1964

Site: intestine

Host: Micronycteris hirsuta

Dist.: Costa Rica

Record: Caballero 1964b

Limatulum limatulum (Braun, 1900) Travassos, 1921

Syn.: Distomum limatulum Braun, 1900

Site: intestine

Host: Natalus stramineus mexicanus

Dist.: Mexico Record: Caballero 1943a

Limatulum mcdanieli Brooks and Coggins, 1983

Site: stomach

Host: Myotis lucifugus

Dist.: WI

Record: Brooks and Coggins 1983

Limatulum oklahomensis Macy, 1932

Site: intestine

Hosts: Myotis grisescens (2)

Tadarida brasiliensis cynocephala (1)

T. b. mexicana (3)

Dist.: KS, OK, TX
Records: 1. Macy 1932 (KS,OK); 2. Ubelaker 1966 (KS); 3. Martin 1976 (TX)

Limatulum spp.

Site: stomach

Host: Myotis lucifugus

Dist.: WI

Record: Coggins et al. 1982

Maxbraunium tubiporum (Braun, 1900) Caballero and Ma, 1942

Syn.: Distomum tubiporum Braun, 1900

Site: intestine

Hosts: Nycteris borealis

$N$. b. borealis

Dist.: Mexico

Pteronotus parnellii rubiginosus

Record: Caballero and Cerecero 1942

Ochoterenatrema breckenridgei (Macy, 1936)

Syn.: Lecithodendrium breckenridge Macy, 1936

Site: small intestine

Host: Eptesicus fuscus (2)

Dist.: MN

Pipistrellus sp. (1)

Record: 1. Macy 1936c; 2. Lotz and Font 1983

Ochoterenatrema diminutum (Chandler, 1938)

Syn.: Limatulum diminutum Chandler, 1938

Ochoterenatrema caballeroi Freitas, 1957

Prosthodendrium diminutum

Chandler, 1938

Site: small intestine

Host: Eptesicus fuscus (4)

Myotis nigricans nigricans (2)

Nycticeius humeralis $(1,3)$

Dist.: IA, Panama, TX, WI

Records: 1. Chandler 1938 (TX); 2. Caballero

1964 (Panama); 3. Ubelaker and Kunz 1971

(IA); 4. Lotz and Font 1983 (WI)

Ochoterenatrema labda Caballero, 1943

Site: small intestine

Hosts: Myotis nigricans nigricans (3)

Natalus stramineus mexicanus (1)

Tadarida brasiliensis $(1,2)$

T. b. cynocephala (5)

T. b. mexicana $(4,5)$

Dist.: LA, Mexico, NM, Panama, TX

Records: 1. Caballero 1943a (Mexico); 2

Jameson 1959 (TX); 3. Caballero 1964a

(Panama); 4. Cain 1966 (NM); 5. Martin

1976 (LA,TX) 
Ochoterenatrema travassosi (Macy, 1938)

Syn.: Prosthodendrium travassosi Macy, 1938

Site: small intestine

Host: Eptesicus fuscus

Dist.: MN, WI

Records: Macy 1938 (MN); Lotz and Font 1983 (WI)

Ototrema schildti Font, 1978

Site: intestine

Hosts:Myotis keenii (2)

Dist.: WI

$M$. lucifugus $(1,3)$

Records: 1. Font 1978; 2. Coggins et al. 1981, 3. 1982

Paralecithodendrium carlsbadensis Cain, 1966

Site: small intestine

Host: Tadarida brasiliensis mexicana

Dist.: NM

Record: Cain 1966

Paralecithodendrium chilostomum (Mehlis, 1831)

Syn.: Prosthodendrium oligolecithum Manter and Dubois, 1945

Site: small intestine

Host: Eptesicus fuscus (2)

Myotis californicus (1)

Dist.: NE, WI

Records: 1. Manter and Debus 1945 (NE); 2.

Lotz and Font 1983 (WI)

Paralecithodendrium emollidum (Caballero, 1943)

Syn.: Prosthodendrium emollidum

Caballero, 1943

Site: intestine

Host: Natalus stramineus mexicanus

Dist.: Mexico

Record: Caballero 1943a

Paralecithodendrium macnabi (Macy, 1936)

Syn.: Prosthodendrium duboisi Neiland, 1962

Prosthodendrium macnabi Macy, 1936

Prosthodendrium mizellei Seamster and Stevens, 1948

Site: small intestine

Host: Eptesicus fuscus $(1,2,3,5,6)$

Lasionycteris noctivagans (6)

Myotis lucifugus $(5,7)$

Nycteris cinerea (4)

Dist.: AK, BC, IN, Mexico, MN, WI

Records: 1. Macy 1936a (MN), 2. 1936b (MN);

3. Seamster and Stevens 1948 (IN); 4.

Caballero and Zerecero 1951 (Mexico); 5.
Neiland 1962 (AK); 6. Webster and Casey

1973 (BC); 7. Lotz and Font 1983 (MN,WI)

Paralecithodendrium naviculum (Macy, 1936)

Lotz and Font 1983

Syn.: Limatulum scabrum Caballero, 1940

Prosthodendrium naviculum

Macy, 1936

Prosthodendrium paeminosum

Caballero, 1943

Prosthodendrium scabrum (Caballero, 1940) Caballero, 1943

Prosthodendrium tetralobulatum

Caballero, 1943

Site: small intestine

Host: Balantiopteryx plicata (5)

Eptesicus fuscus $(1,2,12)$

Mustela vison (10)

Myotis sodalis (8)

$M$. volans longicrus (11)

Nycteris cinerea (6)

$N$. intermedia floridana (4)

Procyon lotor (9)

P. l. lotor (7)

Tadarida brasiliensis (3)

Dist.: BC, GA, KY, Mexico, MI, MN, MS, $\mathrm{OH}, \mathrm{WI}$

Records: 1. Macy 1936a (MN), 2. 1936b (MN);

3. Caballero 1940 (Mexico); 4. Byrd and

Macy 1942 (MS); 5. Caballero 1943b

(Mexico); 6. Caballero and Zerecero 1951

(Mexico); 7. Williams $1961(\mathrm{OH}) ; 8$. Williams

1962 (KY); 9. Harkema and Miller 1964

(GA); 10. Lang and Dronen 1972 (MI); 11.

Webster and Casey 1973 (BC); 12. Lotz and

Font 1983 (MN,WI)

Paralecithodendrium nokomis (Macy, 1937)

Syn.: Prosthodendrium nokomis, Macy, 1937

Site: small intestine

Host: Eptesicus fuscus (2)

Nycteris borealis (1)

Dist.: IA, WI

Records: 1. Blankespoor and Ulmer 1970

(IA); 2. Lotz and Font 1983 (WI)

Paralecithodendrium swansoni (Macy, 1936) Lotz and Font, 1983

Syn.: Prosthodendrium swansoni Macy, 1936

Site: small intestine

Host: Eptesicus fuscus $(5,8)$

Myotis grisescens (4)

M. lucifugus $(1,2,5,6,7)$

Nycteris borealis (5)

Tadarida brasiliensis cynocephala (3)

Dist.: BC, IA, KS, LA, MN, WI 
Records: 1. Macy 1936a (MN), 2. 1936b (MN); 3. Byrd and Macy 1942 (LA); 4. Nickel and Hansen 1967 (KS); 5. Blankespoor and Ulmer 1970 (IA); 6. Webster and Casey 1973 (BC); 7. Coggins et al. 1982 (WI); 8. Lotz and Font 1983 (MN,WI)

Paralecithodendrium transversum (Byrd and Macy, 1942) Lotz and Font, 1983

Syn.: Prosthodendrium transversum Byrd and Macy, 1942

Prosthodendrium volaticum

Blankespoor and Ulmer, 1972

Site: small intestine

Host: Eptesicus fuscus $(4,8)$

Myotis keenii $(5,6)$

$M$. sodalis (2)

Nycteris borealis $(1,3)$

$N$. b. borealis (1)

Dist.: IA, KY, MN, TN, WI

Records: 1. Byrd and Macy $1942(\mathrm{TN}) ; 2$.

Williams 1962 (KY): 3. Kunz 1968 (IA); 4.

Blankespoor and Ulmer 1972 (IA); 5.

Blankespoor and Szymusiak 1974 (IA); 6.

Coggins et al. 1981 (WI), 7. 1982 (WI); 8.

Lotz and Font 1983 (MN,WI)

Prosthodendrium alaskensis Neiland, 1962

Site: intestine

Host: Myotis lucifugus

Dist.: AK

Record: Neiland 1962

Prosthodendrium longiforme Bhalerao, 1926

Site: intestine

Host: Eptesicus fuscus fuscus

Dist.: KS

Record: Nickel and Hansen 1967

Prosthodendrium silvai (Travassos, 1928)

Site: intestine

Host: Nycteris cinerea

Dist.: BC

Record: Webster and Casey 1973

Prosthodendrium singularium Byrd and Macy, 1942

Site: small intestine

Host: Nycteris intermedia floridana

Dist.: MS

Record: Byrd and Macy 1942

Prosthodendrium spp.

Site: intestine

Hosts:Eptesicus fuscus $(1,2)$

Lasionycteris noctivagans (2)

Myotis lucifugus (2)
Nycteris borealis (1)

$N$. cinerea (2)

Dist.: BC, IA

Record: 1. Blankespoor and Ulmer 1970 (IA);

2. Webster and Casey 1973 (BC)

Tremajoannes buckleyi Saoud, 1964

Site: small intestine

Hosts: Tadarida brasiliensis mexicana

Dist.: NM

Record: Cain 1966 (NM)

\section{UNIDENTIFIED LECITHODENDRIIDAE}

Lecithodendriidae gen. spp.

Site: intestine

Host: Myotis californicus caurinus

Dist.: OR

Record: Macy 1947

FAMILY MICROPHALLIDAE Ward, 1901

Carneophallus turgidus Leigh, 1958

Site: small intestine

Hosts:Procyon lotor $(1,2,4,5)$

$$
\begin{aligned}
& \text { P. l. litoreus (6) } \\
& \text { P. l. solutus (3) }
\end{aligned}
$$

Dist.: FL, GA, NC, SC, TX

Records: 1. Leigh 1958 (FL); 2. Jordan and Hayes 1959 (GA); 3. Harkema and Miller 1962 (SC), 4. 1964 (NC,SC); 5. Schaffer et al. 1981 (GA,TX); 6. Price and Harman 1983 (GA)

Gynaecotyla adunca (Linton, 1905)

Site: small intestine

Hosts:Procyon lotor $(2,3)$

$$
\text { P. l. solutus (1) }
$$

Dist.: GA, NC, SC

Records: 1. Harkema and Miller 1962 (SC), 2.

1964 (NC,SC); 3. Schaffer et al. 1981 (GA)

Maritrema prolixum Caballero and Gei, 1961

Site: intestine

Host: Metachirops opossum fuscogriseus

Dist.: Costa Rica

Record: Caballero and Gei 1961

Maritreminoides nettae (Gower, 1936) Rankin, 1939

Site: small intestine

Hosts: Didelphis virginiana (2)

Procyon lotor $(1,3)$

Dist.: GA, MN, NC, SC 
Records: 1. Harkema and Miller 1964

(GA,NC,SC); 2. Miller and Harkema 1970

(NC); 3. Larson and Scharf 1975 (MN)

Maritreminoides spp.

Site: small intestine

Hosts: Procyon lotor (2)

P. l. solutus (1)

Dist.: NC, SC

Records: 1. Harkema and Miller 1962 (SC), 2. 1964 (NC,SC)

Microphallus aspalacis Rausch, 1962

Site: small intestine

Host: Neurotrichus gibbsii

Dist.: OR

Record: Rausch 1962

Microphallus enhydrae Rausch and Locker, 1951

Site: small intestine

Host: Enhydra lutris

Dist.: AK

Record: Rausch and Locker 1951

Microphallus pirum (Afanas'ev, 1941)

Site: intestine

Host: Enhydra lutris

Dist.: AK

Record: Rausch 1953

Microphallus spp.

Site: small intestine

Hosts: Procyon lotor (1)

P. l. hirtus (2)

Dist.: $\mathrm{OH}$, Sask

Record: 1. Rausch 1946a (OH); 2. Hoberg

and McGee 1982 (Sask)

Pseudomaritrema beolopolskoi Caballero 1964

Site: intestine

Host: Micronycteris hirsuta

Dist.: Costa Rica

Record: Caballero 1964b

Pseudospelotrematoides spp.

Site: small intestine

Host: Sus scrofa

Dist.: GA

Record: Babero et al. 1959

FAMILY NANOPHYETIDAE Dollfus, 1939

Nanophyetus salmincola (Chapin, 1926) Chapin, 1927

Syn.: Nanophyes salmincola Chapin, 1926

Site: small intestine
Hosts: Canis latrans (4)

C. l. lestes (1)

Felis concolor (6)

Lutra canadensis (4)

Lynx rufus (4)

L. r. fasciatus (1)

Mustela erminea (4)

$M$. vison $(2,3,4)$

Procyon lotor (4)

P. l. pacificus (1)

Rattus norvegicus (4)

Spilogale putorius (4)

Vulpes vulpes (4)

Zalophus californianus (5)

Dist.: Ont, OR, WA

Records: 1. Cram 1926 (WA); 2. Anonymous 1930 (Ont); 3. Senger and Neiland 1955

(OR); 4. Schlegel et al. 1968 (OR,WA); 5.

Stroud and Dailey 1978 (OR); 6. Kistner et al. $1979(\mathrm{OR})$

FAMILY PARAGONIMIDAE Dollfus, 1939

Paragonimus kellicotti (Ward, 1908)

Site: lungs

Hosts:Canis latrans $(19,20)$

Didelphis marsupialis $(8,17)$

Lynx rufus $(7,8,14,21)$

Mephitis mephitis $(8,15,16,19,20)$

Mustel vison $(1,2,3,4,5,9,11,12,19,20)$

Ondatra zibethicus (18)

Procyon lotor $(8,10)$

Vulpes vulpes $(6,19,20)$

Mink (13)

Dist.: GA, OH, Ont, KY, MD, MI, MN, NC, WI, WV

Records: 1. Ameel 1931 (MI), 2. 1934 (WI); 3.

La Rue and Ameel 1937 (MI,OH); 4.

Sealander 1943 (MI); 5. Erickson 1946 (MN),

6. 1950 (MN); 7. Jordan and Byrd 1958 (GA);

8. McKeever 1958 (GA); 9. Gesinski et al. $1964(\mathrm{OH}) ; 10$. Harkema and Miller 1964

(GA,NC); 11. Miller and Harkema 1964

(NC); 12. Beckett and Gallicchio $1966(\mathrm{OH})$; 13. Fyvie 1966 (Ont); 14. Miller and

Harkema 1968 (NC); 15. Bemrick and

Schlotthauer $1971(\mathrm{MN}) ; 16$. Alexander et al. 1972 (MD); 17. Feldman et al. 1972 (NC); 18.

Harley $1972(\mathrm{KY}) ; 1$ 19. Presidente and

Ramsden 1975 (Ont); 20. Ramsden and

Presidente 1975 (Ont); 21. Watson et al. 1981 (GA,WV)

Paragonimus mexicanus Miyazaki and Ishii, 1968

Syn.: Paragonimus ecuadoriensis

Paragonimus inca 
Paragonimus peruvianus Miyazaki, Ibanez, and Miranda, 1969

Site: lungs

Hosts:Didelphis marsupialis $(1,2)$

D. virginiana californica $(3,4,5,6)$

Felis pardalis (2)

Metachirops opossum fuscogriseus (2)

M. o. pallidus $(4,6,7)$

Procyon lotor (2)

Urocyon cinereoargenteus (2)

Dist.: Costa Rica, Mexico

Records: 1. Miyazaki and Ishii 1968

(Mexico); 2. Brenes et al. 1980 (Costa Rica);

3. Miyazaki, Kifune and Lamothe-Argumedo

1980 (Mexico); 4. Lamothe-Argumedo 1981

(Mexico); 5. Lamothe-Argumedo et al. 1981

(Mexico); 6. Lamothe-Argumedo 1984

(Mexico); 7. Lamothe-Argumedo et al. 1985

(Mexico)

Remarks: Brenes et al. (1980) considered $P$. peruvianus a synonym of $P$. mexicanus.

Brenes et al. (1980) redescribed the material he called and reported in 1968 as

Paragonimus sp.. See supplementary

reading for Brenes et al. (1968).

Paragonimus rudis (Diesing, 1850) Braun, 1899

Site: lungs

Hosts:Didelphis marsupialis (4)

D. virginiana (1)

Felis onca (3)

Mephitis macroura macroura (1)

Metachirops opossum (4)

M. o. fuscogriseus (2)

Nasua nasua (4)

Procyon lotor (3)

Dist.: Costa Rica, GA, Guatemala, NC, Panama

Records: 1. Caballero 1946 (Guatemala); 2.

Caballero and Gei 1961 (Costa Rica); 3.

Harkema and Miller 1964 (GA, NC); 4.

Thatcher 1967 (Panama)

Paragonimus westermani (Kerbert, 1878)

Site: lungs

Host: Didelphis virginiana

Dist.: TN

Records: Byrd and Reiber 1942a, 1942b;

Byrd, Reiber, et al. 1942

Paragonimus spp.

Site: lungs

Hosts:Didelphis marsupialis $(5,6)$

Felis onca (7)

F. pardalis (7)

Felidae gen. spp. (7)

Mustela vison (2)
Ondatra zibethicus (1)

Procyon lotor lotor (4)

Mink (3)

Dist.: Belize, Canada, Mexico, MI, Ont

Records: 1. Ameel 1932 (MI); 2. Swales 1933

(Ont): 3. Cameron 1937b (Canada); 4.

Rausch and Tiner 1949 (MI); 5. Mazzotti and

Miyazaki 1965 (Mexico); 6. Miyazaki and

Ishii 1968 (Mexico); 7. Patton et al. 1986

(Belize)

Remarks: Raush and Tiner (1949) considered

$P$. l. lotor to be an accidental host for

Paragonimus spp.

FAMILY PLAGIORCHIIDAE Ward, 1917

Neoglyphe locellus (Kossacks, 1910)

Syn.: Opisthioglyphe locellus Kossacks, 1910

Site: intestine

Hosts:Sorex cinereus

S. palustris

S. vagrans

Dist.: $\mathrm{CO}$

Records: Leiby 1961

Neoglyphe soricis (Pojmanska, 1957)

Site: intestine

Hosts:Sorex cinereus

S. palustris

S. vagrans

Dist.: MT

Record: Hendricks 1973

Plagiorchis elegans (Rudolphi, 1802)

Site: intestine

Hosts: Alopex lagopus (2)

Procyon lotor hirtus (1)

Vulpes vulpes (2)

Dist.: AK, GD, Sask

Record: 1. Hoberg and McGee 1982 (Sask); 2.

Rausch et al. 1983 (AK,GD)

Plagiorchis massino Petrov and Tikhonov, 1927

Site: small intestine

Host: Vulpes vulpes alascensis

Dist.: AK

Record: Babero and Rausch 1952

Plagiorchis micracanthos Macy, 1932

Syn.: Plagiorchis micracanthum Macy, 1931

Site: intestine

Hosts:Eptesicus fuscus (1)

Myotis californicus (2)

$M$. grisescens (4)

$M$. lucifugus $(1,7)$

M. l. occultus (6) 
M. sodalis (3)

Pipistrellus hesperus (7)

P. subflavus subflavus (5)

Dist.: KS, KY, MN, NE, NM, NV

Records: 1. Macy 1932 (MN); 2. Manter and Debus 1945 (NE); 3. Williams 1962 (KY); 4. Ubelaker 1966 (KS); 5. Nickel and Hansen 1967 (NE); 6. Cain and Studier 1972 (NM), 7. 1974 (NM,NV)

Plagiorchis muris (Tanabe, 1922) Yamaguti, 1933

Site: small intestine

Hosts:Mephitis mephitis (3)

Microtus pennsylvanicus (2)

Natalus stramineus mexicanus (1)

Procyon lotor (5)

Sigmodon hispidus (4)

Dist.: Mexico, ND, Nfld, Que, TX

Records: 1. Caballero 1943a (Mexico); 2.

Schad 1954 (Nfld, Que); 3. Dyer 1970 (ND);

4. Rodenberg and Pence 1978 (TX); 5.

Schaffer et al. 1981 (TX)

Plagiorchis noblei Fedorova, 1969

Site: intestine

Host: Ondatra zibethicus

Dist.: Man

Record: McKenzie and Welch 1979

Plagiorchis proximus Barker, 1915

Site: duodenum, small intestine

Hosts: Glaucomys sabrinus (23)

Mustela vison $(2,3)$

Ondatra zibethicus

$(1,3,4,5,6,11,13,14,17,18,19,22)$

O. z. obscurus $(2,3)$

O. z. osoyooensis $(12,21)$

O. z. zibethicus $(8,9,10,15,20)$

Spermophilus franklinii (23)

$S$. richardsonii $(7,23)$

Tamiasciurus hudsonicus (23)

Zapus hudsonius (16)

Dist.: AK, BC, CO, IL, MA, Man, ME, MI, NB, NE, Nfld, NY, OH, Ont, PA, Que, Sask, UT, WI

Records: 1. Barker 1915 (NE); 2. Anonymous 1930 (Ont); 3. Law and Kennedy 1932 (Ont); 4. Ameel 1942 (MI); 5. Rankin 1946 (MA); 6. Rausch 1946b (OH); 7. Rausch and Tiner 1948 (Man); 8. Edwards 1949 (NY); 9.

Rausch and Tiner 1949 (WI); 10. Meyer and Reilly 1950 (ME); 11. Knight 1951 (BC); 12. Ball 1952 (CO); 13. Rausch 1952b (AK); 14.

Sweatman 1952 (Ont); 15. Gilford 1954 (IL); 16. Schad 1954 (Nfld,Que); 17. Dunagan 1957a (AK), 18. 1957b (AK);19. Anderson and Beaudoin 1966 (PA); 20. Beckett and
Gallicchio $1967(\mathrm{OH}) ; 21$. Grundmann and Tsai 1967 (UT); 22. MacKinnon and Burt 1978 (NB); 23. McGee 1980 (Sask); 24. Rigby and Threlfall 1981 (Nfld)

Plagiorchis vespertilionis (Mueller, 1784) Braun, 1900

Site: small intestine

Hosts:Eptesicus fuscus $(5,6)$

E. f. fuscus (4)

Lasionycteris noctivagans (6)

Myotis californicus (6)

$M$. keenii $(7,9)$

$M$. lucifugus $(1,5,6,7,10)$

M. l. lucifugus (4)

$M$. volans longicrus (6)

Nycteris cinerea $(5,6)$

Tadarida brasiliensis $(2,3)$

T. b. cynocephala (8)

T. b. mexicana (8)

Dist.: BC, IA, KS, LA, Mexico, NE, TX, WI Records: 1. Stafford 1905 (not specified); 2.

Caballero 1940 (Mexico), 3. 1943a (Mexico);

4. Nickel and Hansen 1967 (KS,NE); 5.

Blankespoor and Ulmer 1970 (IA); 6.

Webster and Casey 1973 (BC); 7.

Blankespoor and Szymusiak 1974 (IA); 8.

Martin 1976 (LA,TX); 9. Coggins et al. 1981 (WI), 10.1982 (WI)

Plagiorchis vespertilionis parorchis Macy, 1960

Site: small intestine

Host: Myotis lucifugus alascensis

Dist.: WA

Record: Macy 1960 (WA)

Plagiorchis spp.

Site: small intestine

Hosts:Aello megalophylla (4)

Clethrionomys gapperi (3)

C. rutilus dawsoni (2)

Eptesicus fuscus (6)

Lasionycteris noctivagans (6)

Myotis lucifugus (5)

Nycticeius humeralis (1)

Dist.: AK, BC, Nfld, Que, TN, TX

Records: 1. Byrd and Macy $1942(\mathrm{TN}) ; 2$.

Rausch 1952b (AK); 3. Schad 1954

(Nfld,Que); 4. Jameson 1959 (TX); 5.

Neiland 1962 (AK); 6. Webster and Casey 1973 (BC)

\section{FAMILY PROSTHOGONIMIDAE Luhe, 1909}

Mediogonimus ovilacus Woodhead and Malewitz, 1936 
Site: bile ducts, beneath liver capsule

Hosts: Microtus pennsylvanicus pennsylvanicus $(1,2)$ Ondatra zibethicus zibethicus (3)

Dist.: MI, OH

Records: 1. Woodhead and Malewitz 1936

(MI); 2. Rausch and Tiner 1949 (MI); 3.

Beckett and Gallicchio 1967 (OH)

FAMILY TELORCHIIDAE Nicoll, 1924

Telorchis spp.

Site: small intestine

Host: Lutra canadensis

Dist.: AL

Record: Fleming et al. 1977

\section{FAMILY TROGLOTREMATIDAE Braun, 1915}

Sellacotyle vitellosa Sogandares-Bernal, 1961

Site: small intestine

Host: Mustela vison vulgivaga

Dist.: LA

Record: Sogandares-Bernal 1961

Troglotrema mustelae Wallace, 1932

Syn.: Sellacotyle mustelae Wallace, 1934

Site: small intestine

Hosts: Mustela vison $(1,2,3,6)$

Procyon lotor $(4,5)$

Urocyon cinereoargenteus (7)

Dist.: GA, MN, NC, SC, VA

Records: 1. Wallace $1932(\mathrm{MN})$; 2. Wallace 1935 (MN); 3. Erickson 1946 (MN); 4.

Sawyer 1958 (GA); 5. Harkema and Miller 1964 (GA,NC,SC,VA); 6. Miller and

Harkema 1964 (NC), 7. 1968 (GA)

Xiphidiotrema lockerae Senger, 1953

Site: small intestine

Hosts:Sorex bendirii palmeri

S. palustrus navigator

S. vagrans permiliensis

Dist.: MT, OR

Record: Senger 1953

FAMILY UROTREMATIDAE Poche, 1926

Urotrema scabridum (Braun, 1900) Caballero, 1942

Site: small intestine

Hosts: Eptesicus nilssoni (2)

Myotis grisescens $(3,4)$

M. keenii (7)
M. lucifugus $(5,8)$

Natalus stramineus mexicanus (1)

Tadarida brasiliensis (1)

T. b. cynocephala (6)

T. b. mexicana (6)

Dist.: Costa Rica, IA, KS, LA, Mexico, TX, WI

Records: 1. Caballero 1942 (Mexico); 2.

Caballero et al. 1957 (Costa Rica); 3.

Ubelaker 1966 (KS); 4. Nickel and Hansen 1967 (KS); 5. Blankespoor and Ulmer 1970 (IA); 6. Martin 1976 (LA,TX); 7. Coggins et al. 1981 (WI), 8.1982 (WI)

Remarks: Eptesicus propinguus Peters, 1872 is regarded as a synonym of Eptesicus nilssoni (Keyserling and Blasius, 1839) but this species does not occur in the New World (Hall 1981). Therefore, the host record is uncertain.

Urotrema shillingeri Price, 1931

Site: small intestine

Hosts: Nycticeius humeralis (2)

Ondatra zibethicus (1)

O. z. macrodon (3)

Dist.: MD, TX

Records: 1. Price 1931b(MD); 2. Chandler 1938 (TX); 3. Penner 1941 (MD)

\section{ORDER STRIGEIFORMES}

\section{LaRue, 1926}

FAMILY BRACHYLAEMIDAE

Joyeux and Foley, 1930

Brachylaima bravoae (Deloya, 1970)

Syn.: Brachylaemus bravoae Deloya, 1970

Site: small intestine

Host: Liomys pictus pictus

Dist.: Mexico

Record: Deloya 1970

Brachylaima chiapensis Ubelaker and Dailey, 1966

Site: small intestine

Host: Peromyscus guatemalensis guatemalensis

Dist.: Mexico

Record: Ubelaker and Dailey 1966

Brachylaima didelphus Premvati and Bair, 1979

Site: intestine

Host: Didelphis virginiana

Dist.: FL

Record: Premvati and Bair 1979 
Brachylaima dolichodirus Mason, 1953

Site: intestine

Host: Blarina brevicauda

Dist.: NC, TN

Record: 1. Mason $1953(\mathrm{TN}) ;$ 2. Miller et al. 1974

Brachylaima opisthotrias (Lutz, 1895) Dollfus, 1935

Syn.: Distomum opisthotrias Lutz, 1895

Harmostomum opisthotrias (Lutz, 1895) Braun, 1899

Site: small intestine, ileum

Host: Didelphis virginiana $(1,2,3,4,5)$

Didelphis sp. (6)

Dist.: IL, PA, TN, TX, VA

Records: 1. Dickerson 1930 (VA); 2.

Chandler 1932 (TX); 3. Canavan 1934 (PA);

4. Leigh 1940 (IL); 5. Byrd and Reiber 1942a

(TN); 6. Babero 1957 (IL)

Brachylaima opisthotrias virginiana (Dickerson, 1930)

Syn.: Harmostomum opisthotrias virginiana Dickerson, 1930

Site: small intestine

Host: Didelphis virginiana

Dist.: VA

Record: Dickerson 1930

Brachylaima ovatus (Sinitsin, 1931)

Syn.: Ectosiphonus ovatus Sinitsin, 1931

Site: small intestine

Host: Blarina brevicauda

Dist.: IA

Record: Wittrock and Hendrickson 1979

Brachylaima peromysci Reynolds, 1938

Site: small intestine

Hosts:Mus musculus (2)

Peromyscus leucopus (2)

P.l. leucopus (1)

Dist.: KY, MD, VA

Records: 1. Reynolds 1938 (VA); 2. Hall and

Sonnenberg 1955 (KY, MD)

Brachylaima rauschi McIntosh, 1950

Site: intestine

Hosts:Dicrostonyx groenlandicus rubricatus Microtus miurus muriei

Dist.: AK

Record: McIntosh 1950; Rausch 1952a

Brachylaima rhomboideus (Sinitsin, 1931)

Site: stomach, small intestine

Host: Blarina brevicauda

Dist.: CT, IA
Records: Wittrock and Hendrickson 1979

(IA); Huffman and Penner 1981 (CT)

Brachylaima thompsoni (Sinitsin, 1931)

Syn.: Entosiphonus thompsoni (Sinitsin, 1931)

Site: small intestine

Hosts: Blarina brevicauda $(3,4,5,6)$
B. b. brevicauda (2)
B. b. kirtlandi (2)
Peromyscus leucopus leucopus (1)
$P$. $l$. noveboracensis (3)
Sorex cinereus cinerus (2)

Dist.: CT, IA, MI, NC, WI

Records: 1. Harkema 1936 (NC); 2. Rausch and Tiner 1949 (MI,WI); 3. Villella 1953

(MI); 4. Miller et al. 1974 (NC); 5. Wittrock and Hendrickson 1979 (IA); 6. Huffman and Penner 1981 (CT)

Brachylaima virginiana (Dickerson, 1930)

Syn.: Brachylaemus migrans of Sinitzin, 1931

Brachylaimus virginianus Dickerson, 1930

Site: small intestine

Hosts:Dasypus novemcinctus (3)

Didelphis marsupialis (15)

D. virginiana $(2,7,10,12,14)$

D. v. virginiana $(4,11)$

Didelphis sp. $(1,5,6)$

Mephitis mephitis (8)

Mustela vison (9)

Peromyscus leucopus (12)

$P$. l. noveboracensis (4)

Procyon lotor (16)

Dist.: CA, GA, IL, LA, MI, NC, TN, TX, VA, WV

Records: 1. Reynolds 1938(VA); 2. Byrd and Reiber 1942a (GA, TN); 3. Chandler 1946 (TX); 4. Rausch and Tiner 1949 (MI); 5.

Hand and Voge 1952 (CA); 6. Babero 1957

(IL), 7. 1960a (LA), 8. 1960b (GA); 9.

Lumsden and Zischke 1961 (LA); 10. Kaplan

1964 (LA); 11. Holloway 1966 (VA); 12.

Miller and Harkema 1970 (NC); 13. Zenchak and Hall 1971 (WV); 14. Feldman et al. 1972 (NC); 15. Nettles et al. 1975 (GA); 16.

Schaffer et al. 1981 (VA)

Brachylaima spp.

Site: small intestine

Hosts:Microtus pennsylvanicus terraenovae (1) Ochotona collaris (2) Sciurus carolinensis (3)

Dist.: AK, MD, NC, Nfld 
Records: 1. Rausch 1952b (Nfld); 2. Dunagan

1957a (AK); 3. Davidson 1976 (MD, NC)

Ityogonimus scalopi Turner and McKeever, 1980

Site: intestine

Host: Scalopus aquaticus

Dist.: SC

Record: Turner and McKeever 1980

Panopistus pricei Sinitsin, 1931

Site: small and large intestine

Hosts:Blarina brevicauda $(2,3,4,5,6)$

B. b. brevicauda (1)

B. b. kirtlandi (1)

Dist.: CT, IL, IA, MI, NC, WI

Records: 1. Rausch and Tiner 1949 (MI,WI);

2. Villella 1954 (MI); 3. Levin 1957 (IL); 4.

Miller et al. 1974 (NC); 5 . Wittrock and

Hendrickson 1979 (IA); 6. Huffman and

Penner 1981 (CT)

Postharmostomum helicis (Leidy, 1847) Robinson, 1949

Syn.: Distoma helicis Leidy, 1847 Postharmostomum laruei, 1934

Site: caecum

Host: Peromyscus maniculatus (2)

Tamias striatus lysteri (1)

Dist.: MI, Que

Record: 1. McIntosh 1934 (MI); 2. Schad

1956 (Que)

Postharmostomum noveboracensis McIntosh, 1937

Site: intestine

Host: Tamias striatus lysteri

Dist.: NY

Record: McIntosh 1937

Scaphiostomum microti (Kruidenier and Gallicchio, 1959) Mas-Coma, Esteban and Valero, 1986

Syn.: Brachylaime microti Kruidenier and Gallicchio, 1959

Site: bile ducts, pancreatic duct

Hosts: Microtus longicaudus baileyi (1) Peromyscus maniculatus sonoriensis $(2,3,4)$

Spermophilus variegatus utah (5)

Dist.: CO, UT

Records: 1. Kruidenier and Gallicchio 1959

(CO); 2. Frandsen and Grundmann 1960

(UT); 3. Grundmann and Frandsen 1960

(UT); 4. Frandsen and Grundmann 1961

(UT); 5. Jenkins and Grundmann 1973 (UT)

Scaphiostomum pancreaticum McIntosh, 1934

Site: pancreatic duct
Hosts:Peromyscus gossypinus

gossypinus (2)

Tamias striatus $(3,4)$

T. s. lysteri (1)

Dist.: GA, MI, Ont, WI

Records: 1. McIntosh 1934 (MI), 2. 1935

(GA); 3. Rausch and Tiner 1948 (WI); 4.

Jensen 1972 (Ont)

FAMILY CYATHOCOTYLIDAE Muhling, 1898

Linstowiella szidati (Anderson, 1944)

Syn.: Cercaria szidati, 1944

Site: small intestine

Host: Didelphis virginiana

Dist.: LA

Record: Lumsden and Winkler 1962

FAMILY DIPLOSTOMIDAE Poirier, 1886

Alaria alata (Goeze, 1782) Krause, 1914

Site: intestine

Host: Vulpes vulpes

Dist.: Man, PEI, Que

Record: Swales 1933 (PEI,Que); Baron 1970

(Man)

Alaria americana (Hall and Wigdor, 1918)

Syn.: Alaria canis LaRue and Fallis, 1936

Site: intestine

Hosts: Canis latrans $(7,10,13)$

C. lupus $(1,5,7,8,9,10,12,15)$

Lynx canadensis (7)

L. rufus $(7,11)$

Martes pennanti (7)

Urocyon cinereoargenteus $(4,11)$

Ursus americanus (15)

Vulpes vulpes $(1,2,4,6,7,8,14,16,17)$

V. v. alaskensis $(3,5)$

Dist.: AK, Alta, MN, NB, NC, NS, Ont, PEI, Que, TX, WI, YT

Records: 1. Law and Kennedy 1932 (Ont); 2.

Swales 1933b (PEI,Que); 3. Dubois and

Rausch 1950 (AK); 4. Erickson 1950 (MN) 5.

Babero and Rausch 1952 (AK); 6. Pearson

1954 exp. (Ont), 7. 1956 (Ont); 8. Dunagan

1957a (AK); 9. Rausch and Williamson 1959

(AK); 10. Holmes and Podesta 1968 (Alta);

11. Miller and Harkema 1968 (NC); 12.

Choquette, Gibson et al. 1973 (YT); 13.

Thornton et al. 1974 (TX); 14. Gilbertson

1977 (MN); 15. Addison et al. 1978 (Ont); 16.

Smith 1978 (NB,NS); 17. Dibble et al. 1983

(WI) 
Alaria americana Hall and Wigdor, 1918

(mesocercaria)

Site: many tissues

Hosts: Lutra canadensis (2)

Mus musculus (2)

Mustela sp.(1,2)

Peromyscus maniculatus $(1,2)$

Dist.: Ont

Records: 1. Pearson 1954 exp., 2. 1956 exp.

Alaria arisaemoides Augustine and Uribe, 1927

Syn.: Alaria oregonensis La Rue and Barone, 1927

Site: intestine

Hosts: Canis latrans $(2,7,8,11)$

C. l. lestes (3)

C. lupus $(9,11)$

Urocyon cinereoargenteus (5)

Vulpes vulpes $(1,4,5,6,10,11,12,13)$

Dist.: Alta, MA, Man, MN, NB, NS, NWT, Ont, OR, WI, YT

Records: 1. Augustine and Uribe 1927 (MA);

2. La Rue and Barone 1927 (OR), 3. 1932

(OR); 4. Law and Kennedy 1932 (Ont); 5.

Rankin 1946 (MA); 6. Pearson 1954 exp.

(Ont); 7. 1956 (Ont); 8. Holmes and Podesta

1968 (Alta); 9. Choquette, Gibson et al. 1973

(NWT,YT); 10. Gilbertson 1977 (MN); 11.

Samuel, Ramalingam et al. 1978 (Man); 12.

Smith 1978 (NB,NS); 13. Dibble et al. 1983

(WI)

Alaria arisaemoides Augustine and Uribe, 1927

(mesocercaria)

Site: many tissues

Hosts: Mus musculus (2)

Mustela sp. (1)

Peromyscus maniculatus $(1,2)$

Ferret (1)

Lab. mice (1)

Dist.: Ont

Records: 1. Pearson 1954 exp. , 2. 1956

Alaria craterum (Barker and Noll, 1915)

Site: duodenum

Hosts: Mustela vison (1)

Ondatra zibethicus (1)

Dist.: Ont

Record: Law and Kennedy 1932

Alaria marcianae (LaRue, 1917) Walton, 1949

Site: small intestine

Hosts: Canis latrans $(2,4,6)$

C. lupus (7)

Lynx canadensis (8)

L. rufus (3)

Mephitis mephitis (1)
Procyon lotor hirtus (5)

Spilogale putorius (1)

Taxidea taxus (1)

Urocyon cinereoargenteus (1)

Vulpes vulpes $(1,5)$

Dist.: Man, MN, MT, Ont, Que, Sask, TX

Records: 1. Johnson 1968 (MN); 2. Samuel,

Ramalingan et al. 1978 (Man); 3. Stone and

Pence 1978 (TX); 4. Pence and Meinzer 1979

(TX); 5. Hoberg and McGee 1982 (Sask); 6.

Seesee et al. 1983 (MT); 7. McNeill et al.

1984 (Que); 8. Smith et al. 1986 (Ont)

Alaria minnesotae Chandler, 1954

Site: small intestine

Host: Mephitis mephitis

Dist.: MN

Record: Chandler 1954

Alaria mustelae Bosma, 1931

Syn.: Alaria canadensis Webster and

Wolfgang, 1956

Alaria dubia Chandler and Rausch, 1946

Alaria intermedia Olivier and Odlaug, 1938

Alaria minuta Chandler and Rausch, 1946

Alaria taxideae Swanson and

Erickson, 1946

Site: small intestine

Hosts: Canis latrans (9)

Gulo luscus $(14,16)$

Martes americana (27)

M. a. actuosa (17)

$M$. pennanti (24)

Mephitis mephitis $(5,7,13,20,25)$

M. m. nigra $(6,8,11)$

Mustela erminea (25)

M. e. cicognanii $(5,7)$

$M$. frenata $(5,7,12,25)$

$M$. f. noveboracensis $(3,7)$

M.vison $(1,2,3,4,5,12,18,19,25)$

M. v. letifera $(7)$

Mustela spp. (1)

Ondatra zibethicus $(2,10,14,15,26)$

Spilogale putorius $(5,7,9,25)$

Taxidea taxus $(4,7,21,22,25)$

Urocyon c. cinereoargenteus (6)

Vulpes vulpes $(2,23)$

Dist.: AK, IA, MA, Man, MD, MI, MN, NB, NC, ND, NS, NWT, Ont, OR, Que, WI

Records: 1. Bosma 1931 (MI); 2. Law and

Kennedy 1932 (Ont); 3. Bosma 1934 (MI); 4.

Chandler and Rausch 1946 (MI); 5. Erickson 1946 (MN); 6. Rankin 1946 (MA); 7.

Swanson and Erickson 1946 (MN); 8. Rausch 
and Tiner 1949 (MI); 9. Erickson 1950 (MN); 10. Sweatman 1952 (Ont); 11. Goldberg 1954 (MD); 12. Senger and Neiland 1955 (OR); 13. Webster and Wolfgang 1956 (Que); 14.

Dunagan 1957a (AK), 15. 1957b (AK); 16.

Dubois and Rausch 1960 (AK); 17. Holmes

1963 (NWT); 18. Miller and Harkema 1964

(NC); 19. Dorney and Lauerman 1969 (WI);

20. Dyer 1970 (ND); 21. Leiby et al. 1971

(ND); 22. Wittrock and Ulmer 1974 (IA); 23.

Smith 1978b (NB,NS); 24. Dick and Leonard 1979 (Man); 25. Johnson 1979 (MN); 26.

McKenzie and Welch 1979 (Man); 27. Poole et al. 1983 (Man)

Alaria mustelae Bosma, 1931 (mesocercaria)

Site: lungs, spleen, musculature

Host: Spermophilus franklinii

Dist.: Sask

Record: McGee 1980 (Sask)

Alaria mustelae Bosma, 1931 (metacercaria)

Site: muscle

Host: Peromyscus leucopus noveboracensis

Dist.: MI

Record: Bosma 1934

Alaria nasuae LaRue and Townsend, 1927

Site: ceca

Host: Nasua nasua

Dist.: DC

Records: LaRue and Townsend 1927, 1932

Remarks: The specimens for LaRue and

Townsend's (1927) study came from material

deposited in a collection housed at the

Bureau of Animal Industry, Washington,

DC. The collection site was not given in the paper.

Alaria spp.

Site: small intestine

Hosts:Canis latrans $(1,7)$

C. lupus (8)

Gulo luscus $(4,6)$

Lynx canadensis (5)

Martes pennanti (2)

Mephitis mephitis avia (3)

Dist.: AK, Alta, NWT, Ont, OR, TX, WI

Records: 1. Cram 1926 (OR); 2. Anonymous

1930 (Ont); 3. Rausch and Tiner 1949 (WI);

4. Rausch 1959 (AK); 5. Zyll DeJong 1966

(Alta,NWT); 6. Addison and Boles 1978

(NWT); 7. Pence and Meinzer 1979 (TX); 8.

Archer et al. 1986 (WI)

Didelphodiplostomum variabile (Chandler, 1932)

Dubois, 1945
Syn.: Diplostomum variabile (Chandler, 1932)

Proalaria variabilis Chandler, 1932

Site: intestine

Host: Didelphis virginiana $(1,2,3,4,6,7,8)$

Didelphis sp. (5)

Dist.: FL, GA, IL, NC, TN, TX

Records: 1. Chandler 1932 (TX); 2. Leigh

1940 (IL); 3. Byrd and Reiber 1942a (IL,TN);

4. Babero 1957 (IL), 5. 1960a (GA); 6. Miller

and Harkema 1970 (NC); 7. Feldman et al.

1972 (NC); 8. Premvati and Bair 1979 (FL)

Diplostomum mergi Dubois, 1932

Site: intestine

Host: Ondatra zibethicus obscurus

Dist.: Nfld

Record: Rigby and Threlfall 1981

Enhydridiplostomum alarioides (Dubois, 1937)

Dubois, 1944

Site: small intestine

Hosts: Lutra canadensis $(1,3,4)$

Dist.: AL, GA, NC

Records: 1. Sawyer 1961 (GA); 2. Miller and

Harkema 1964 (NC), 3. 1968 (NC); 4.

Flemming et al. 1977 (AL)

Fibricola cratera (Barker and Noll, 1915) Dubois, 1932

Syn.: Hemistomum craterum Barker and Noll, 1915

Fibricola laruei Miller, 1940

Fibricola nana Chandler and Rausch, 1946

Site: intestine

Hosts:Didelphis virginiana $(3,16)$

D. v. virginiana (5)

Mephitis mephitis $(7,10)$

M. m. nigra (6)

Mustela vison $(5,11)$

Ondatra zibethicus (1)

O. z. osoyoosensis (14)

O. z. zibethicus $(8,13)$

Peromyscus maniculatus bairdii (6)

Procyon lotor $(2,7,9,11,12,17)$

P. l. lotor (6)

Tamiasciurus hudsonicus (4)

T.h. loquax (4)

Taxidea taxus (15)

Dist.: FL, GA, IA, IL, LA, MI, NC, NE, OH, Que, SC, TN, TX, UT, WI, WY

Records: 1. Barker 1915 (NE); 2. Miller 1940

(Que); 3. Byrd and Reiber 1942a (GA,TN); 4.

Rausch and Tiner 1948 (MI); 5. Read 1948

(TX); 6. Rausch and Tiner 1949 (MI,WI); 7.

Dubois and Rausch 1950 (WY); 8. Gilford 
1954 (IL); 9. Hoffman 1955 (unspecified); 10.

Webster and Wolfgang 1956 (Que); 11.

Lumsden and Zischke 1961 (LA); 12.

Harkema and Miller 1964 (FL,NC,SC); 13.

Beckett and Gallicchio $1967(\mathrm{OH}) ; 14$.

Grundmann and Tsai 1967 (UT); 15.

Wittrock and Ulmer 1974 (IA); 16. Premvati and Bair 1979 (FL); 17. Schaffer et al. 1981

(FL,GA,TX)

Remarks: Rausch and Tiner (1948)

considered $T$. h. loquax an accidental host for

F. nana.

Fibricola lucida (LaRue and Bosma, 1927) Dubois and Rausch, 1950

Syn.: Neodiplostomum lucidum LaRue and

Bosma, 1927

Site: duodenum, small intestine

Hosts: Didelphis virginiana $(1,2,5,6,7)$

Didelphis sp. (4)

Mustela vison (3)

Dist.: FL, GA, IL, LA, TN, TX

Records: 1. LaRue and Bosma 1927 (TX); 2.

Byrd and Reiber 1942 (GA,TN); 3. Read 1948

(TX); 4. Babero 1957 (IL); 5. Lumsden and

Zischke 1961 (LA); 6. Kaplan 1964 (LA); 7.

Premvati and Bair 1979 (FL)

Fibricola texensis Chandler, 1942

Site: small intestine

Hosts: Procyon lotor $(3,4,5,7)$
P. l. lotor $(1,2)$
P. l. solutus $(6)$

Dist.: GA, SC, TN, TX

Records: 1. Chandler 1942a (TX), 2. 1942b

(TX); 3. Dubois and Rausch 1950 (TX); 4.

Babero and Shepperson 1958 (GA); 5. Sawyer

1958 (GA); 6. Harkema and Miller 1962

(SC); 7. Bafundo et al. 1980 (TN)

Fibricola spp.

Site: small intestine

Hosts: Procyon lotor (1)

Sus scrofa (2)

Dist.: GA

Records: 1. Babero and Shepperson 1958; 2.

Babero et al. 1959

Parallelorchis diglossus Harkema and Miller, 1961

Site: small intestine

Host: Procyon lotor

Dist.: FL, TN

Records: 1. Harkema and Miller 1961 (FL), 2.

1964 (FL); 3. Bafundo et al. 1980 (TN); 4.

Schaffer et al. 1981 (FL)
Pharyngostomoides adenocephala Beckerdite, Miller and Harkema, 1971

Site: small intestine

Hosts: Procyon lotor $(1,2,4)$

P. l. hirtus (3)

Dist.: FL, NC, Ont, Sask, TX

Records: 1. Beckerdite et al. 1971 (NC); 2.

Schaffer et al. 1981 (FL,TX); 3. Hoberg and

McGee 1982 (Sask); 4. Butterworth and

Holmes 1984 (Ont)

Pharyngostomoides ovale Chandler and Rausch, 1946

Site: intestine

Hosts: Procyon lotor (2)

$$
\text { P. l. lotor (1) }
$$

Dist.: FL, MI

Records: 1. Rausch and Tiner 1949 (MI); 2.

Loftin 1960 (FL)

Pharyngostomoides procyonis Harkema, 1942

Site: small intestine

Hosts: Procyon lotor $(3,4,6,7,8,9,10,12,13)$

$$
\begin{aligned}
& \text { P. l. lotor }(1,2) \\
& P . \text { l. solutus }(5) \\
& \text { Ursus americanus (11) }
\end{aligned}
$$

Dist.: FL, GA, NC, Ont, SC, TN, TX, VA

Records: 1. Chandler 1942a (TX); 2.

Harkema 1942 (NC); 3. Babero and

Shepperson 1958 (GA); 4. Sawyer 1958 (GA);

5. Harkema and Miller 1962 (SC), 6. 1964

(FL, GA, NC, SC, VA); 7. Harris et al. 1967

(NC); 8. Beckerdite et al. 1971 (NC,TX); 9.

Bafundo et al. 1980 (TN); 10. Schaffer et al.

1981 (FL, NC, TX, VA); 11. Conti et al. 1983

(FL); 12. Butterworth and Holmes 1984

(Ont); 13. Miller 1984 (NC)

Procyotrema marsupiformis Harkema and Miller, 1959

Site: gall bladder, pancreatic duct

Hosts: Procyon lotor $(2,3,4,6)$

$$
\begin{aligned}
& P \text {. l. lotor (1) } \\
& \text { Urocyon cinereoargenteus (5) }
\end{aligned}
$$

Dist.: MD, NC

Records: 1. Harkema and Miller 1959 (NC), 2. 1964 (NC); 3. Miller and Harkema 1964 (NC); 4. Locke and Brown 1965 (MD); 5.

Miller and Harkema 1968 (NC); 6. Schaffer et al. $1981(\mathrm{NC})$

\section{FAMILY HASSTILESIIDAE Hall, 1916}

Hasstilesia tricolor (Stiles and Hassall, 1894) Hall, 1916

Syn.: Distoma tricolor Stiles and Hassall, 1894 
Hasstilesia novicia

Hasstilesia texensis Chandler, 1929

Monostomum sp. of Curtice (1892)

Site: small intestine

Hosts: Lepus americanus (1)

L. californicus texianus (3)

Sylvilagus aquaticus (17)

S. a. aquaticus (7)

S. floridanus $(7,12,18,21,22,23,24,25)$

S. f. alacer (3)

S. f. mallurus $(4,15,19)$

S. f. mearnsii $(6,11,13,14)$

S. nutallii $(1,16)$

S. palustris $(19,20)$

S. p. palustris (15)

S. transitionalis $(20)$

Vulpes vulpes (15)

Cottontail $(5,8,9)$

Jack Rabbit (2)

Rabbit $(2,10)$

Varying hare (5)

Dist.: AL, AR, CT, DC, FL, GA, IA, KS, KY, LA, MD, MI, MN, MO, MS, NC, NJ, NY, OH, PA, RI, SC, TN, TX, VA, WI, WV

Records: 1. Stiles and Hassall 1894b

(DC,VA); 2. Hall 1916 (DC,MD,NY,TX,VA);

3. Chandler 1929 (TX); 4. Harkema 1936

(NC); 5. Bump 1937 (NY); 6. Morgan and

Waller 1940 (IA); 7. Byrd and Reiber 1942b

(MS,TN); 8. Bell and Chalgren 1943

(AL,CT,KS,MD,MO,NJ,NY,PA,RI,VA,WV);

9. Llewellyn and Handley 1945 (VA); 10.

Ward 1946 (MS); 11. Erickson 1947 (MN);

12. Moore and Moore 1947 (AL); 13. Rausch

and Tiner 1949 (MI,WI); 14. Rowan 1955

(NY); 15. Robinson 1959 (GA,NY,OH); 16.

Marker and Cheng 1960 (PA); 17. Lumsden

and Zischke 1961 (LA); 18. Franklin et al.

1966 (KS); 19. Stringer et al. 1969 (NC); 20.

Eckerlin 1973 (CT,FL); 21. Jacobson et al.

1974 (VA); 22. Jacobson et al. 1978 (VA); 23.

Andrews et al. 1980 (AL,AR,MD,MS,

NC,SC,VA); 24. Wiggins et al. 1980 (PA); 25.

Strohlein and Christensen 1983 (KY)

Remarks: Curtice (1892) originally described this trematode as Monostomum sp. from

Lepus americanus. The Monostomum sp. was subsequently redescribed as Hasstilesia

tricolor (Stiles and Hassall, 1894) Hall, 1916.

Hasstilesia spp.

Site: intestine

Host: Cottontail rabbit

Dist.: NY

Records: Bump 1940, 1941, 1942

\section{FAMILY LEUCOCHLORIDIOMORPHIDAE}

Travassos and Kohn, 1966

Ptyalincola ondatrae Wootton and Murrell, 1967

Site: ducts of salivary glands and oral cavity

Host: Ondatra zibethicus

Dist.: MI

Record: Wootton and Murrell 1967

\section{FAMILY SCHISTOSOMATIDAE \\ Stiles and Hassall, 1898}

Heterobilharzia americana Price, 1929

Site: liver, mesenteric and portal veins

Hosts: Canis latrans (9)

C. latrans $x$ C. rufus (9)

C. rufus (9)

Castor canadensis (12)

Didelphis virginiana (7)

Lynx rufus floridanus (1)

Myocastor coypus $(4,5,7)$

Odocoileus virginianus (8)

Procyon lotor $(2,3,4,5,6,7,8,10,11,13)$

Sylvilagus aquaticus $(4,5)$

Rabbit (7)

Dist.: FL, GA, LA, NC, SC, TX

Records: 1. Price 1929 (FL), 2. 1943 (TX); 3.

Miller and Harkema 1960 (NC); 4. Malek et

al. 1961 (FL,LA); 5. Lee 1962 (LA); 6.

Harkema and Miller 1964 (FL,NC); 7.

Kaplan 1964 (LA); 8. Byrd et al. 1967

(GA,SC); 9. Custer and Pence 1981 (LA,TX);

10. Schaffer et al. 1981 (FL,NC,TX); 11.

Kocan et al. 1982 (FL); 12. Fedynick et al.

1986 (TX); 13. Short and Grossman 1986

(TX)

Remarks: Price (1929) collected his

specimens from a Lynx sp., possibly L. uinta,

from the National Zoological Park in

Washington, DC.

Heterobilharzia spp.

Site: mesenteric or portal vein

Host: Myocastor coypus bonariensis

Dist.: LA

Record: Babero and Lee 1961

Schistosomatium douthitti (Cort, 1914) Price, 1929

Site: portal and mesenteric veins, liver, spleen

Hosts: Erethizon dorsatum (7)

Microtus pennsylvanicus $(5,9)$

M. p. pennsylvanicus (2)

Ondatra zibethicus $(1,3,10,11)$

O. z. zibethicus $(4,6)$ 
Peromyscus leucopus (9)

Zapus hudsonius $(8,9)$

Dist.: IL, Man, MI, MN, NB, OH, Ont

Records: 1. Penner 1938 (MI,MN); 2. Price

1939 (MI); 3. Ameel 1942 (MI); 4. Gilford

1954 (IL); 5. Farley 1962 (Man); 6. Beckett and Gallicchio $1967(\mathrm{OH}) ;$ 7. Choquette, Broughton et al. 1973 (Ont); 8. Carmichael and Muchlinski 1980 (MI); 9. Zajac and Williams 1980 (MI); 10. MacKinnon and Burt 1978 (NB); 11. McKenzie and Welch 1979 (Man)

\section{UNIDENTIFIED SCHISTOSOMATIDAE}

\section{Schistosomatidae gen. spp.}

Site: intestinal contents

Host: Myocastor coypus bonariensis

Dist.: LA

Record: Babero and Lee 1961

\section{UNIDENTIFIED DIGENEA}

Braunina cordiformis (Wolf)

Site: pyloric and second stomachs

Host: Tursiops nesarnack

Dist.: CA, FL

Records: Johnston and Ridgway 1969 (CA);

Woodard et al. 1969 (FL)

Digenea gen. spp.

Site: small intestine, spinal cord at the foramen magnum

Hosts: Eptesicus fuscus (5)

Globicephala sieboldii (3)

Myotis keenii (5)

M. lucifugus (5)

$M$. velifer incautus (2)

Peromyscus maniculatus (1)

Pipistrellus subflavus (5)

Tadarida brasiliensis (2)

Zalophus californianus (4)

Dist.: CA, IN, TX

Records: 1. Schad 1956 (Que); 2. Jameson 1959 (TX); 3. Norris 1966 (CA); 4. Migaki et al. 1971 (unspecified); 5. Whitaker and Mumford 1971 (IN) 
HOST - PARASITE LIST 


\section{CLASS MAMMALIA \\ SUBCLASS THERIA \\ INFRACLASS EUTHERIA \\ ORDER ARTIODACTYLA}

FAMILY BOVIDAE

Bison bison (Linnaeus)

American bison

FASCIOLIDAE

Fasciola hepatica (MT,WY)

Bos taurus Linnaeus

Feral cattle

FASCIOLIDAE

Fasciola hepatica (GA)

PARAMPHISTOMIDAE

Calicophoron microbothrioides (GA)

\section{FAMILY CERVIDAE}

Alces alces (Linnaeus)

Moose

FASCIOLIDAE

Fascioloides magna (Alta,BC,Man,MN,Ont)

PARAMPHISTOMIDAE

Paramphistomum cervi

(MN,Nfld,Ont,Que,Sask)

$P$. liorchis (Ont)

Paramphistomum spp. (Alta,MN)

Zygocotyle lunata (Alta)

Alces alces americana (Clinton)

SYN.: Alces americanus (Clinton)

FASCIOLIDAE

Fascioloides magna (MN)

PARAMPHISTOMIDAE

Paramphistomum cervi (MN)

Cervus elaphus (Linnaeus)

FASCIOLIDAE

Wapiti

Fasciola hepatica (MT)

Fascioloides magna (Alta,BC,Ont,OR,Que,)

Cervus elaphus nelsoni Bailey

SYN.: Cervus canadensis nelsoni Bailey

FASCIOLIDAE

Fascioloides magna (OR)

Cervus elaphus roosevelti Merriam

SYN.: Cervus canadensis roosevelti Merriam FASCIOLIDAE

Fascioloides magna (WA)

Cervus unicolor

Sambar deer

PARAMPHISTOMIDAE

Paramphistomum liorchis (FL)

Odocoileus hemionus (Rafinesque)

FASCIOLIDAE
Fasciola hepatica (WA)

Odocoileus hemionus columbianus (Richardson)

FASCIOLIDAE

Fasciola hepatica (BC,CA)

Fascioloides magna (Alta,BC,NY,OR)

Odocoileus hemionus hemionus (Rafinesque)

FASCIOLIDAE

Fascioloides magna (Alta,BC)

Odocoileus virginianus (Zimmerman) White-

SYN.: Cariacus virginianus tailed deer (Zimmerman)

DICROCOELIDAE

Dicrocoelium dendriticum (NY)

FASCIOLIDAE

Fasciola hepatica (WA)

Fascioloides magna

(AL,Alta,AR,BC,FL,GA,LA,MI,MN,MS,MT,N

C,NY,Ont,OR, Que,SC,TN,TX,WI)

PARAMPHISTOMIDAE

Paramphistomum cervi (AL)

P. liorchis (AL,FL, GA,LA,SC)

Paramphistomidae gen. spp. (FL)

Zygocotyle lunata (MI)

SCHISTOSOMATIDAE

Heterobilharzia americana (SC)

Odocoileus virginianus borealis Miller

FASCIOLIDAE

Fascioloides magna (MN)

Odocoileus virginianus leucurus (Douglas)

FASCIOLIDAE

Fascioloides magna (OR)

Odocoileus spp.

FASCIOLIDAE

Fasciola hepatica (CA,MT)

Fascioloides magna (MT,NC,NY,Ont)

PARAMPHISTOMIDAE

Paramphistomum liorchis (AL,GA)

Rangifer tarandus caribou (Gmelin) Caribou

SYN.: Rangifer caribou sylvestris Richardson FASCIOLIDAE

Fascioloides magna (MN,Que)

FAMILY SUIDAE

Sus scrofa

DIPLOSTOMIDAE

Feral swine

Fibricola spp. (GA)

FASCIOLIDAE

Fasciola hepatica (GA)

Fascioloides magna (TX)

MICROPHALLIDAE

Pseudospelotrematoides spp. (GA) 
FAMILY TAYASSUIDAE

Dicotyles tajacu angulatus Cope Collared peccary FASCIOLIDAE

Fascioloides magna (TX)

\section{ORDER CARNIVORA FAMILY CANIDAE}

Alopex lagopus (Linnaeus)

Arctic fox

SYN.: Vulpes lagopus

ECHINOSTOMATIDAE

Echinoparyphium spp. (GD)

HETEROPHYIDAE

Apophallus venustus (DC)

Cryptocotyle concavum (GD)

Phagicola longa (DC)

$P$. nana (DC)

PLAGIORCHIIDAE

Plagiorchis elegans (AK,GD)

Canis latrans Say

Coyote

DIPLOSTOMIDAE

Alaria americana (Alta,Ont,TX)

A. arisaemoides (Alta,Man,Ont,OR)

A. marcianae (Man,MT,TX)

A. mustelae (MN)

Alaria spp. (OR,TX)

NANOPHYETIDAE

Nanophyetus salmincola (OR)

OPISTHORCHIIDAE

Metorchis conjunctus (Alta)

PARAGONIMIDAE

Paragonimus kellicotti (Ont)

SCHISTOSOMATIDAE

Heterobilharzia americana (LA,TX)

Canis latrans lestes Merriam

SYN.: Canis lestes Merriam

DIPLOSTOMIDAE

Alaria arisaemoides (OR)

NANOPHYETIDAE

Nanophyetus salmincola (WA)

Canis lupus Linnaeus

Gray wolf

DIPLOSTOMIDAE

Alaria americana (AK,Alta,Ont, YT)

A. arisaemoides (Man, NWT, YT)

A. marcianae (Que)

Alaria sp. (WI)

OPISTHORCHIIDAE

Metorchis conjunctus (Alta,Sask)

Canis rufus Audubon and Bachman Red wolf

SCHISTOSOMATIDAE

Heterobilharzia americana (LA,TX)
Canis rufus $\mathrm{x}$ Canis latrans

DICROCOELIIDAE

Eurytrema procyonis (LA,TX)

SCHISTOSOMATIDAE

Heterobilharzia americana (LA,TX)

Urocyon cinereoargenteus (Schreber) Gray fox DICROCOELIIDAE

Eurytrema procyonis (MD,NC)

DIPLOSTOMIDAE

Alaria americana (MN,NC)

A. arisaemoides (MA)

A. marcianae (MN)

Procyotrema marsupiformis (NC)

HETEROPHYIDAE

Euryhelmis squamula (GA)

PARAGONIMIDAE

Paragonimus mexicanus (Costa Rica)

TROGLOTREMATIDAE

Troglotrema mustelae (GA)

Urocyon cinereoargenteus cinereoargenteus

(Schreber)

DIPLOSTOMIDAE

Alaria arisaemoides (MA)

A. mustelae (MA)

Vulpes vulpes Linnaeus

Red fox

SYN.: Vulpes fulva Desmarest

DICROCOELIIDAE

Eurytrema procyonis (NY)

DIPLOSTOMIDAE

Alaria alata (Man,PEI,Que)

A. americana

(AK,MN,NB,NS,Ont,PEI,Que,WI)

A. arisaemoides

(MA,Man,MN,NB,NS,Ont,WI)

A. marcianae (MN,Sask)

A. mustelae (NB,NS,Ont)

ECHINOSTOMATIDAE

Echinoparyphium spp. (IL)

Echinostoma revolutum (NB,NS)

HASSTILESIIDAE

Hasstilesia tricolor $(\mathrm{OH})$

HETEROPHYIDAE

Cryptocotyle lingua (NB,NS)

Phagicola longa (Ont,Que)

NANOPHYETIDAE

Nanophyetus salmincola (OR)

OPISTHORCHIIDAE

Metorchis conjunctus (NB,NS)

PARAGONIMIDAE

Paragonimus kellicotti (MN,Ont)

PLAGIORCHIIDAE

Plagiorchis elegans (AK)

Vulpes vulpes alascensis Merriam

SYN.: Vulpes fulva alascensis Merriam

DIPLOSTOMIDAE 
Alaria americana (AK)

PLAGIORCHIIDAE

Plagiorchis massino (AK)

CANIDAE OF UNDETERMINED SPECIES

Canidae gen. spp.

Fox

DICROCOELIIDAE

Eurytrema procyonis (NY)

\section{FAMILY FELIDAE}

Felis concolor Kerr

NANOPHYETIDAE

Nanophyetus salmincola (OR)

Felis onca Mearns

Jaguar

SYN.: Panthera onca (Mearns)

PARAGONIMIDAE

Paragonimus rudis (GA,NC)

Paragonimus spp. (Belize)

Felis pardalis (Linnaeus)

Ocelot

PARAGONIMIDAE

Paragonimus mexicanus (Costa Rica)

Paragonimus spp. (Belize)

Lynx canadensis Kerr

DIPLOSTOMIDAE

Lynx

Alaria americana (Ont)

A marcianae (Ont)

Alaria sp.(Alta,NWT)

OPISTHORCHIIDAE

Opisthorchis tenuicollis (PA)

Lynx rufus (Schreber)

Bobcat

SYN.: Felis rufa Schreber

DIPLOSTOMIDAE

Alaria americana (NC,Ont)

A. marcianae (TX)

NANOPHYETIDAE

Nanophyetus salmincola (OR)

PARAGONIMIDAE

Paragonimus kellicotti (GA,NC,WV)

$P$. rudis (NC)

Lynx rufus fasciatus Rafinesque

SYN.: Lynx fasciatus fasciatus Rafinesque

NANOPHYETIDAE

Nanophyetus salmincola (WA)

Lynx rufus floridanus Rafinesque

SCHISTOSOMATIDAE

Heterobilharzia americana (FL)

FELIDAE OF UNDETERMINED SPECIES
Paragonimus spp. (Belize)

FAMILY MUSTELIDAE

Enhydra lutris (Linnaeus)

CAMPULIDAE

Orthosplanchnus fraterculus (AK)

HETEROPHYIDAE

Phocitrema fusiforme (AK)

Pricetrema zalophi (AK)

MICROPHALLIDAE

Microphallus enhydrae (AK)

M. pirum (AK)

Gulo luscus (Linnaeus)

SYN.: Gulo Gulo

DIPLOSTOMIDAE

Alaria mustelae (AK)

Alaria spp. (AK,NWT)

Lutra canadensis (Schreber)

River otter

SYN.: Lutra canadensis vaga Bangs

DIPLOSTOMIDAE

Alaria americana (Ont)

Enhydridiplostomum alarioides (AL,GA,NC)

ECHINOSTOMATIDAE

Baschkirovitrema incrassatum

(AL,GA,NC,NY)

Euparyphium melis (MI)

NANOPHYETIDAE

Nanophyetus salmincola (OR,WA)

TELORCHIIDAE

Telorchis spp. (AL)

Martes americana (Turton)

Marten

DIPLOSTOMIDAE

Alaria mustelae (Man)

Martes americana actuosa (Osgood)

DIPLOSTOMIDAE

Alaria mustelae (NWT)

Martes pennanti (Erxleben)

DIPLOSTOMIDAE

Alaria americana (Ont)

A. mustelae (Man)

Alaria spp. (Ont)

ECHINOSTOMATIDAE

Echinostoma spp. (Ont)

OPISTHORCHIIDAE

Metorchis conjunctus (Man)

Mephitis macroura macroura Lichtenstein

PARAGONIMIDAE

Hooded skunk

Paragonimus rudis (Guatemala)

Mephitis mephitis Schreber

Striped skunk

Fisher

Felidae gen. spp.

PARAGONIMIDAE 
Brachylaima virginiana (GA)

DIPLOSTOMIDAE

Alaria marcianae (MN)

A. minnesotae (MN)

A. mustelae (MN,ND,Que)

Fibricola cratera (Que,WY)

HETEROPHYIDAE

Apophallus spp. (Que)

Euryhelmis pyriformis (Que)

PARAGONIMIDAE

Paragonimus kellicotti (GA,IA,MD,MN,Ont)

PLAGIORCHIIDAE

Plagiorchis muris (ND)

PSILOSTOMIDAE

Psilostomum spp. (LA)

Mephitis mephitis avia Bangs Striped skunk

DIPLOSTOMIDAE

Alaria spp. (WI)

Mephitis mephitis nigra (Peale and Palisot de

Beauvois)

SYN.: Mephitis nigra (Peale and Palisot de

Beauvois)

DIPLOSTOMIDAE

A. mustelae (MA,MD,MI)

Fibricola cratera (MI)

Mustela erminea (Linnaeus)

Ermine

DIPLOSTOMIDAE

Alaria mustelae (MN)

NANOPHYETIDAE

Nanophyetus salmincola (OR)

Mustela erminea cicognanii Bonaparte

SYN.: Mustela cicognanii Bonaparte

DIPLOSTOMIDAE

Alaria mustelae (MN)

ECHINOSTOMATIDAE

Euparyphium melis (MN)

Mustela frenata Lichenstein Long-tailedweasel

DIPLOSTOMIDAE

Alaria mustelae (MN,OR)

Mustela frenata noveboracensis (Emmons)

SYN.: Mustela noveboracensis noveboracensis

(Emmons)

DIPLOSTOMIDAE

Alaria musteleae (MI,MN)

Mustela vison Schreber

Mink

ALLASSOGONOPORIDAE

Cephalophallus obscurus (MI,OR)

BRACHYLAEMIDAE

Brachylaema virginiana (LA)

DIPLOSTOMIDAE

Alaria craterum (Ont)

A. mustelae (MI,MN,NC,Ont,OR,WI)

Enhydridiplostomum alarioides (NC)
Fibricola cratera (LA,TX)

F. lucida (TX)

Procyotrema marsupiformis (NC)

ECHINOSTOMATIDAE

Baschkirovitrema incrassatum (NC)

Euparyphium melis (MI,MN,Ont)

Euparyphium beaveri (LA,NC,WI)

$E$. inerme (Ont)

HETEROPHYIDAE

Euryhelmis monorchis (MI,OH,WI)

$E$. pacificus $(\mathrm{OR})$

E. squamula (NC,OR)

Metagonimoides oregonensis (MI,OR,NC)

LECITHODENDRIIDAE

Paralecithodendrium naviculum (MI)

NANOPHYETIDAE

Nanophyetus salmincola (Ont,OR)

OPISTHORCHIIDAE

Parametorchis complexus (Ont)

PARAGONIMIDAE

Paragonimus kellicotti

(MI,MN,NC,OH,Ont,WI)

$P$. rudis (NC)

Paragonimus spp. (Ont)

PLAGIORCHIIDAE

Plagiorchis proximus (Ont)

RHOPALIASIDAE

Gyrosoma singularis (LA)

Rhopalias macracanthus (LA)

TROGLOTREMATIDAE

Troglotrema mustelae (MN,NC)

Mustela vison letifera Hollister

DIPLOSTOMIDAE

A. mustelae (WI)

ECHINOSTOMATIDAE

Euparyphium melis (WI)

Mustela vison vulgivaga (Bangs)

TROGLOTREMATIDAE

Sellacoytle vitellosa (LA)

Mustela spp.

DICROCOELIIDAE

Dicrocoelium dendriticum (PEI)

DIPLOSTOMIDAE

Alaria americana (Ont)

A. arisaemoides (Ont)

A. mustelae (MI)

Spilogale putorius (Linnaeus)

DIPLOSTOMIDAE

Alaria marcianae (MN)

A. mustelae (MN)

NANOPHYETIDAE

Nanophyetus salmincola (OR)

Taxidea taxus (Schreber)

Badger

DIPLOSTOMIDAE

Alaria marcianae (MN)
Spotted skunk 
A. mustelae (IA,MN,ND)

Fibricola cratera (IA)

ECHINOSTOMATIDAE

Euparyphium melis (IA,ND)

Euparyphium spp. (MN)

\section{MUSTELIDAE OF \\ UNDETERMINED SPECIES}

Skunk

PSILOSTOMIDAE

Ribeiroia ondatrae (FL)

\section{FAMILY PROCYONIDAE}

Nasua nasua (Linnaeus)

SYN.: Nasua narica (Linnaeus)

DIPLOSTOMIDAE

Alaria nasuae (DC)

PARAGONIMIDAE

Paragonimus rudis (Panama)

Procyon lotor (Linnaeus)

BRACHYLAEMIDAE

Brachylaima virginiana (VA)

DICROCOELIIDAE

Eurytrema procyonis

(CT,GA,MD,NC,NY,TN,TX,VA)

Lyperosomum sinuosum (SC,TE)

DIPLOSTOMIDAE

Fibricola cratera

(FL,GA,LA,NC,Que,SC,TX,WY)

F. texensis (GA,TN,TX)

Fibricola spp. (GA)

Parallelorchis diglossus (FL,TN)

Pharyngostomoides adenocephala

(FL,NC,Ont,TX)

$P$. ovale (FL)

P. procyonis (FL,GA, NC,Ont,SC,TN,TX,VA)

Procyotrema marsupiformis (MD,NC)

ECHINOSTOMATIDAE

Echinostoma revolutum $(\mathrm{OH})$

Euparyphium beaveri (GA,NC,SC,TN)

Stephanoprora spinosa (GA)

HETEROPHYIDAE

Apophallus venustus

(Can,GA,NC,Ont,SC,VA)

Ascocotyle ampullacea (NC)

A. leighi (SC)

A. pachycystis (FL)

Euryhelmis squamula (GA,NC,TN)

Metagonimoides oregonensis

(GA,NC,OR,TN,VA)

Phagicola diminuta (NC,SC)

P. longa (NC,SC)

LECITHODENDRIIDAE

Paralecithodendrium naviculum (GA)

Coati

Racoon
MICROPHALLIDAE

Carneophallus turgidus (FL,GA,NC,SC,TX)

Gynaecotyla adunca (GA,NC,SC)

Maritreminoides nettae (GA, MN,NC,SC)

Maritreminoides spp. (NC,SC)

Microphallus spp. $(\mathrm{OH})$

NANOPHYETIDAE

Nanophyetus salmincola (OR)

OPISTHORCHIDAE

Metorchis conjunctus (VA)

Parametorchis complexus (NC,VA)

PARAGONIMIDAE

Paragonimus kellicotti (GA)

$P$. mexicanus (Costa Rica)

$P$. rudis $(\mathrm{GA}, \mathrm{NC})$

PLAGIORCHIIDAE

Plagiorchis muris (TX)

PSILOSTOMIDAE

Gyrosoma singularis (GA,SC)

Ribeiroia ondatrae (FL)

SCHISTOSOMATIDAE

Heterobilharzia americana

(FL,GA,LA,NC,TX)

TROGLOTREMATIDAE

Troglotrema mustelae (GA,NC,SC,VA)

Procyon lotor crassidens Hollister

DICROCOELIIDAE

Robertdigenea dollfusi (Costa Rica)

Procyon lotor hirtus Nelson and Goldman

MICROPHALLIDAE

Microphallus spp. (Sask)

PLAGIORCHIIDAE

Plagiorchis elegans (Sask)

DIPLOSTOMIDAE

Alaria marcianae (Sask)

Pharyngostomoides adenocephala (Sask)

Procyon lotor litoreus Nelson and Goldman

MICROPHALLIDAE

Carneophallus turgidus (GA)

Procyon lotor lotor (Linnaeus)

DICROCOELIIDAE

Eurytrema procyonis (TX)

DIPLOSTOMIDAE

Fibricola cratera $(\mathrm{MI}, \mathrm{WI})$

F. texensis (TX)

Pharyngostomoides ovale (MI)

$P$. procyonis (NC,TX)

Procyotrema marsupiformis (NC)

HETEROPHYIDAE

Euryhelmis monorchis (MI)

Metagonimoides oregonensis $(\mathrm{OH})$

LECITHODENDRIIDAE

Paralecithodendrium naviculum $(\mathrm{OH})$

PARAGONIMIDAE

Paragonimus spp. (MI)

Procyon lotor pacificus Merriam

SYN.: Procyon psora pacifica Merriam 


\section{HETEROPHYIDAE}

Metagonimoides oregonensis (OR)

NANOPHYETIDAE

Nanophyetus salmincola (WA)

Procyon lotor solutus Nelson and Goldman

DICROCOELIIDAE

Lyperosomum sinuosum (SC)

DIPLOSTOMIDAE

Fibricola texensis (SC)

Pharyngostomoides procyonis (SC)

HETEROPHYIDAE

Ascocotyle leighi (SC)

Phagicola diminuta (SC)

P. longa (SC)

MICROPHALLIDAE

Carneophallus turgidus (SC)

Gynaecotyla adunca (SC)

Maritreminoides spp. (SC)

\section{FAMILY URSIDAE}

Ursus americanus Pallas

Black bear

DIPLOSTOMIDAE

Alaria americana (Ont)

Pharyngostomoides procyonis (FL)

Ursus arctos Linnaeus

ECHINOSTOMATIDAE

Echinostoma revolutum (MT,WY)

\section{ORDER CETACEA \\ FAMILY BALAENOPTERIDAE}

Balaenoptera borealis Lesson

Sei whale

CAMPULIDAE

Lecithodesmus spinosus (BC)

NOTOCOTYLIDAE

Ogmogaster antarctica (BC)

$\mathrm{O}$. plicatus $(\mathrm{BC})$

Balaenoptera physalus (Linnaeus) Fin whale or CAMPULIDAE

Fin-backed whale

Lecithodesmus goliath $(\mathrm{BC})$

NOTOCOTYLIDAE

Ogmogaster antarctica (CA)

O. plicatus (BC,CA)

O. trilineatus $(\mathrm{CA})$

Balaenoptera sp.

NOTOCOYTLIDAE

Ogmogaster plicatus (CA)

\section{FAMILY DELPHINIDAE}

Delphinus delphis Linnaeus Common dolphin SYN.: Delphinus bairdi Dall
CAMPULIDAE

Campula palliata (Costa Rica)

C. rochebruni (CA)

Campulidae gen. spp. (CA)

NASITREMATIDAE

Nasitrema delphini (CA)

$N$. globicephalae (CA)

$N$. stenosoma (CA)

Nasitrema spp. (CA)

Globicephala melaena (Trail)

CAMPULIDAE

Orthosplanchnus arcticus (Nfld)

Long-finned pilot whale

Globicephala sieboldii Gray

Digenea gen. spp. (CA)

Short-finned

pilot whale

Lagenorhynchus obliquidens Gill

Pacific

CAMPULIDAE

white-sided dolphin

Zalophotrema spp. (CA)

NASITREMATIDAE

Nasitrema globicephalae (CA)

Nasitrema spp. (CA)

Lissodelphis borealis (Peale) Northern rightNASITREMATIDAE

Nasitrema globicephalae (CA)

Nasitrema spp. (CA)

Phocoena phocoena (Linnaeus) Harbor porpoise

SYN.: Phocaena vomerina (Gill)

CAMPULIDAE

Campula oblongata (Nfld,OR,WA)

Hadwenius mironovus (OR)

$H$. nipponicus (OR,WA)

Phocoenoides dalli (True)

Dall's porpoise

CAMPULIDAE

Campula oblongata (CA)

NASITREMATIDAE

Nasitrema dalli (CA)

Nasitrema spp. (CA)

Steno bredanensis Lesson Rough-toothed dolphin

CAMPULIDAE

Campula palliata (FL)

Snythesium tursionis (FL)

HETEROPHYIDAE

Pholeter gastrophilus (FL)

Tursiops gillii Dall Gill's bottle-nosed dolphin HETEROPHYIDAE

Pholeter gastrophilus (unspecified) 
Tursiops nesarnack Bonnaterre Lacepede's SYN.: Tursiops bottle-nosed dolphin truncatus (Montagu)

CAMPULIDAE

Campula palliata (FL)

Zalophotrema hepaticum (CA)

HETEROPHYIDAE

Pholeter gastrophilus (FL)

NASITREMATIDAE

Nasitrematidae gen. spp. (unspecified)

UNIDENTIFIED DIGENEA

Braunina cordiformis (CA,FL)

\section{FAMILY ESCHRICHTIDAE}

Eschrichtius gibbosus (Erxleben) Gray whale SYN.: Eschrichtius robustus (Lilljeborg)

\section{CAMPULIDAE}

Lecithodesmus goliath (CA)

NOTOCOTYLIDAE

Ogmogaster antarctica (CA)

$O$. pentalineatus (AL,CA)

\section{FAMILY MONODONTIDAE}

Delphinapterus leucas (Pallas) White whale CAMPULIDAE

Hadwenius seymouri (AK)

\section{ORDER CHIROPTERA}

FAMILY EMBALLONURIDAE

Balantiopteryx plicata Peters Peter's bat SYN.: Balantiopteryx ochoterenai Martinez and Villa

LECITHODENDRIIDAE

Paralecithodendrium naviculum (Mexico)

\section{FAMILY MOLOSSIDAE}

Tadarida brasiliensis Geoffroy

DICROCOELIIDAE

Brazilian free-

Dicrocoelium rileyi (TX)

LECITHODENDRIIDAE

Ochoterenatrema labda (Mexico,TX)

Paralecithodendrium scabrum (Mexico)

PLAGIORCHIIDAE

Plagiorchis vespertilionis (Mexico)

UROTREMATIDAE

Urotrema scabridum (Mexico)

TREMATODA GEN. SPP.

Trematoda spp. (TX)

Tadarida brasiliensis cynocephala (Le Conte)

SYN.: Tadarida cynocephala (Le Conte)

ALLASSOGONOPORIDAE
Allassogonoporus marginalis (LA)

DICROCOELIIDAE

Conspicuum icteridorum (LA)

Dicrocoelium rileyi (KS,LA,OK)

LECITHODENDRIIDAE

Limatulum oklahomensis (KS,OK)

Ochoterenatrema labda (LA)

Paralecithodendrium swansoni (LA)

PLAGIORCHIIDAE

Plagiorchis vespertilionis (LA)

UROTREMATIDAE

Urotrema scabridum (LA)

Tadarida brasiliensis mexicana (Saussure)

DICROCOELIIDAE

Conspicuum icteridorum (TX)

Dicrocoelium rileyi (NM,OK,TX)

Platynosomum beltrani (Mexico)

LECITHODENDRIIDAE

Acanthatrium nycteridis (TX)

Limatulum oklahomensis (TX)

Ochoterenatrema labda (NM,TX)

Paralecithodendrium carlsbadensis (NM)

Tremajoannes buckleyi (NM)

PLAGIORCHIIDAE

Plagiorchis vespertilionis (TX)

UROTREMATIDAE

Urotrema scabridum (TX)

\section{FAMILY NATALIDAE}

Natalus stramineus mexicanus Miller Mexican funnel-eared bat

SYN.: Natalus mexicanus Miller

LECITHODENDRIIDAE

Limatulum limatulum (Mexico)

Ochoterenatrema labda (Mexico)

Paralecithodendrium emollidum (Mexico)

PLAGIORCHIIDAE

Plagiorchis muris (Mexico)

UROTREMATIDAE

Urotrema scabridum (Mexico)

\section{FAMILY PHYLLOSTOMATIDAE}

Aello megalophylla (Peters)Peter's ghost-faced bat SYN.: Mormoops megalophylla Peters

SYN.: Mormoops megalophylla senicula Rehn PLAGIORCHIIDAE

Plagiorchis spp. (TX)

Micronycteris hirsuta (Peters) Hairy largeANENTEROTREMATIDAE eared bat

Anenterotrema freitasi (Costa Rica)

LECITHODENDRIIDAE

Castroia silvai (Costa Rica)

Limatulum istmicus (Costa Rica)

MICROPHALLIDAE 
Pseudomaritrema beolopolskoi (Costa Rica)

Pteronotus parnellii rubiginosus (Wagner)

SYN.: Chilonycteris rubiginosus Parnell's Wagner mustached bat

LECITHODENDRIIDAE

Maxbraunium tubiporum (Mexico)

\section{FAMILY VESPERTILIONIDAE}

Eptesicus fuscus (Beauvois)

ALLASSOGONOPORIDAE

Big brown bat

Allassogonoporus marginalis (WI)

A. vespertilionis $(\mathrm{OR})$

DICROCOELIIDAE

Dicrocoelium rileyi (IA)

LECITHODENDRIIDAE

Acanthatrium alicatai (DC)

A. eptesici (BC,DC,MN,WI)

A. microcanthum (MN,WI)

A. oligacanthum (WI)

A. pipistrelli (IA,MN,WI)

Glyptoporus noctophilus (MN)

Ochoterenatrema breckenridgei (MN)

O. diminutum (WI)

O. travassosi (MN,WI)

Paralecithodendrium chilostomum (WI)

P. macnabi (BC,IN, MN, WI)

$P$. naviculum (MN,WI)

P. nokomis (WI)

P. swansoni (IA,MN,WI)

$P$. transversum (IA,MN,WI)

$P$. vespertilionis (IA)

$P$. volaticum (IA)

Prosthodendrium spp. (BC,IA)

PLAGIORCHIIDAE

Plagiorchis micracanthos (MN)

$P$. vespertilionis $(\mathrm{BC}, \mathrm{IA})$

Plagiorchis spp. (BC)

TREMATODA GEN. SPP.

Trematoda spp. (IN)

Eptesicus fuscus fuscus (Beauvois)

LECITHODENDRIIDAE

Acanthatrium amphidymum (VA)

A. beuschleini (VA)

A. eptesici (VA)

A. lunatum (KY,OH)

A. nycteridis (VA)

A. oligacanthum (VA)

A. pipistrelli $(\mathrm{KY}, \mathrm{OH})$

Lecithodendrium spp. (VA)

Prosthodendrium longiforme (KS)

PLAGIORCHIIDAE

Plagiorchis vespertilionis (KS)

Eptesicus nilssoni (Keyserling and Blasius)

UROTREMATIDAE

Urotrema scabridum (Costa Rica)
Lasionycteris noctivagans (Le Conte) SilverLECITHODENDRIIDAE haired bat

Acanthatrium nycteridis (BC)

Paralecithodendrium macnabi (BC)

Prosthodendrium spp. (BC)

PLAGIORCHIIDAE

Plagiorchis vespertilionis (BC)

Plagiorchis spp. (BC)

Myotis californicus (Audubon and Bachman)

LECITHODENDRIIDAE California myotis

Paralecithodendrium chilostomum (NE)

PLAGIORCHIIDAE

Plagiorchis micracanthos (NE)

$P$. vespertilionis $(\mathrm{BC})$

Myotis californicus caurinus Miller

ALLASSOGONOPORIDAE

Allassogonoporus vespertilionis (OR)

LECITHODENDRIIDAE

Acanthatrium oregonense (OR)

Limatulum gastroides (OR)

Lecithodendriidae gen. spp. (OR)

Myotis evotis evotis (Allen) Long-eared myotis LECITHODENDRIIDAE

Acanthatrium oregonense (OR)

Myotis grisescens Howell

ALLASSOGONOPORIDAE

Allassogonoporus marginalis (KS)

LECITHODENDRIIDAE

Acanthatrium spp. (KS)

Limatulum oklahomensis (KS)

Paralecithodendrium swansoni (KS)

PLAGIORCHIIDAE

Plagiorchis micracanthos (KS)

UROTREMATIDAE

Urotrema scabridum (KS)

Myotis keenii (Merriam)

Keen's myotis

LECITHODENDRIIDAE

Limatulum gastroides (WI)

Ototrema schildti (WI)

Paralecithodendrium transversum (IA,WI)

PLAGIORCHIIDAE

Plagiorchis vespertilionis (IA,WI)

UROTREMATIDAE

Urotrema scabridum (WI)

TREMATODA GEN. SPP.

Trematoda spp. (IN)

Myotis lucifugus (Le Conte) Little brown myotis ALLASSOGONOPORIDAE

Allassogonoporus marginalis (IA,WI)

A. vespertilionis (OR)

Myotitrema asymmetrica (MN) 
LECITHODENDRIIDAE

Acanthatrium beuschleini (VA)

A. oregonense (OR)

A. umbraculatum $(\mathrm{OH})$

Glyptoporus noctophilus (MN)

Gyrabascus brevigastrus (WI)

Lecithodendrium anticum (unspecified)

L. posticum (unspecified)

Limatulum gastroides (IA, MN,WI)

L. mcdanieli (WI)

Limatulum spp. (WI)

Ototrema schildti (WI)

Paralecithodendrium swansoni

(BC,IA,MN,WI)

P. macnabi (AK)

P. transversum (WI)

Prosthodendrium alaskensis (AK)

Prosthodendrium spp. (BC)

PLAGIORCHIIDAE

Plagiorchis micracanthos (MN,NM)

$P$. vespertilionis (BC,IA, WI)

Plagiorchis spp. (AK)

UROTREMATIDAE

Urotrema scabridum (IA,WI)

TREMATODA GEN. SPP.

Trematoda spp. (IN)

Myotis lucifugus alascensis Miller

PLAGIORCHIIDAE

Plagiorchis vespertilionis parorchis (WA)

Myotis lucifugus lucifugus (Le Conte)

LECITHODENDRIIDAE

Acanthatrium nycteridis (MA)

Limatulum gastroides (MA)

PLAGIORCHIIDAE

Plagiorchis vespertilionis (NE)

Myotis lucifugus occultus Hollister

PLAGIORCHIIDAE

Plagiorchis micracanthos (NM)

Myotis nigricans nigricans (Schinz) Black myotis

LECITHODENDRIIDAE

Limatulum costarricensis (Panama)

Ochoterenatrema diminutum (Panama)

O. labda (Panama)

Myotis sodalis Miller and Allen Indiana myotis

ALLASSOGONOPORIDAE

Allassogonoporus marginalis (KY)

LECITHODENDRIIDAE

Acanthatrium pipistrelli (KY)

Limatulum gastroides (KY)

Paralecithodendrium naviculum (KY)

P. transversum (KY)

PLAGIORCHIIDAE

Plagiorchis micracanthos (KY)
Myotis velifer incautus (Allen)

ALLASSOGONOPORIDAE

Allassogonoporus marginalis (KS)

DICROCOELIIDAE

Dicrocoelium rileyi $(\mathrm{KS})$

TREMATODA GEN. SPP.

Trematoda spp. (TX)

Myotis volans longicrus (True)

LECITHODENDRIIDAE

Paralecithodendrium naviculum (BC)

PLAGIORCHIIDAE

Plagiorchis vespertilionis (BC)

Nycteris borealis (Mueller)

Red Bat

SYN.: Lasiurus borealis (Mueller)

DICROCOELIIDAE

Dicrocoelium lasiuri (DC)

LECITHODENDRIIDAE

Acanthatrium eptesici (IA)

A. pipistrelli (IA)

Maxbraunium tubiporum (Mexico)

Paralecithodendrium transversum (IA)

$P$. nokomis (IA)

P. swansoni (IA)

Prosthodendrium eptesici (IA)

P. lunatum (IA)

Prosthodendrium spp. (IA)

Nycteris borealis borealis (Mueller)

SYN.: Lasiurus borealis borealis (Mueller)

SYN.: Vespertilio lasiurus Schreber

LECITHODENDRIIDAE

Paralecithodendrium transversum (TN)

Acanthatrium nycteridis (IL)

Maxbraunium tubiporum (Mexico)

Nycteris cinerea (Palisot de Beauvois) Hoary bat

SYN.: Lasiurus cinereus (Palisot de Beauvois)

LECITHODENDRIIDAE

Limatulum gastroides (BC)

Paralecithodendrium macnabi (Mexico)

P. scabrum (Mexico)

Prosthodendrium silvai (BC)

Prosthodendrium spp. (BC)

PLAGIORCHIIDAE

Plagiorchis vespertilionis (BC,IA)

Nycteris intermedia floridana(Miller) Northern

SYN.: Dasypterus floridanus Miller yellow bat

LECITHODENDRIIDAE

Lecithodendrium spp. (FL)

Paralecithodendrium naviculum (MS)

Prosthodendrium singularium (MS)

Nycticeius humeralis (Rafinesque) Evening bat DICROCOELIIDAE 
Dicrocoelium rileyi (TX)

LECITHODENDRIIDAE

Ochoterenatrema diminutum (IA,TX)

PLAGIORCHIIDAE

Plagiorchis spp. (TN)

UROTREMATIDAE

Urotrema shillingeri (TX)

Pipistrellus hesperus (Allen) Western pipistrelle PLAGIORCHIIDAE

Plagiorchis micracanthos (NV)

Pipistrellus subflavus (Cuvier)Eastern pipistrelle LECITHODENDRIIDAE

Acanthatrium pipistrelli (MN)

TREMATODA GEN. SPP.

Trematoda spp. (IN)

Pipistrellus subflavus subflavus (Cuvier)

PLAGIORCHIIDAE

Plagiorchis micracanthos (NE)

Pipistrellus sp.

LECITHODENDRIIDAE

Ochoterenatrema breckenridgei (MN)

UNIDENTIFIED CHIROPTERA

Eptesicus propinguus

UROTREMATIDAE

Urotrema scabridum (Costa Rica)

Red bat

DICROCOELIIDAE

Dicrocoelium lasiuri (unspecified)

ORDER EDENTATA

FAMILY DASYPODIDAE

Dasypus novemcinctus Peters

BRACHYLAEMIDAE

Nine-banded

Brachylaema virginiana (TX)

\section{ORDER INSECTIVORA}

FAMILY SORICIDAE

Blarina brevicauda (Say) Short-tailed shrew BRACHYLAEMIDAE

Brachylaima dolichodirus (NC,TN)

B. ovatus (IA)

B. rhomboideus (CT,IA)

B. thompsoni (CT,IA,MI,NC)

Panopistus pricei (CT,IL,IA,MI,NC)

DICROCOELIIDAE

Brachylecithum spp. (NC)

Blarina brevicauda brevicauda (Say)

BRACHYLAEMIDAE

Brachylaima thompsoni (WI)

Panopistus pricei (WI)

Blarina brevicauda kirtlandi Bole and Moulthrop BRACHYLAEMIDAE

Brachylaima thompsoni (MI)

Panopistus pricei (MI)

Sorex bendirii palmeri Merriam HETEROPHYIDAE

Pacific

Euryhelmis pacificus (OR)

TROGLOTREMATIDAE

Xiphidiotrema lockerae (MT,OR)

Sorex cinereus Kerr

Masked shrew

PLAGIORCHIIDAE

Neoglyphe locellus (CO)

$N$. soricis (MT)

Sorex cinereus cinereus Kerr

BRACHYLAEMIDAE

Brachylaima thompsoni (WI)

Sorex palustris Richardson

PLAGIORCHIIDAE

Neoglyphe locellus (CO)

$N$. soricis (MT)

Sorex palustris navigator (Baird) TROGLOTREMATIDAE

Xiphidiotrema lockerae (MT,OR)

Sorex vagrans Baird

PLAGIORCHIIDAE

Vagrant shrew

Neoglyphe locellus (CO)

$N$. soricis (MT)

Sorex vagrans permiliensis Jackson

SYN.: Sorex obscurus permiliensis Jackson

TROGLOTREMATIDAE

Xiphidiotrema lockerae (MT,OR)

UNIDENTIFIED SORICIDAE

Shrew

BRACHYLAEMIDAE

Brachylaima thompsoni (MI)

FAMILY TALPIDAE

Neurotrichus gibbsii (Baird)Shrew-mole MICROPHALLIDAE

Microphallus aspalacis (OR)

Scalopus aquaticus (Linnaeus)

BRACHYLAEMIDAE

Itygonimus scalopi (SC) 


\section{ORDER LAGOMORPHA \\ FAMILY LEPORIDAE}

Lepus americanus Erxleben Snowshoe rabbit FASCIOLIDAE

Fasciola hepatica (WA)

HASSTILESIIDAE

Hasstilesia tricolor (DC,VA)

Lepus americanus phaeonotus Allen

ECHINOSTOMATIDAE

Euparyphium melis (MN)

Lepus americanus struthopus Bangs

DICROCOELIIDAE

Dicrocoelium dendriticum (Nfld)

Lepus californicus merriami (Mearns) BlackFASCIOLIDAE tailed jack rabbit

Fasciola hepatica (TX)

Lepus californicus texianus Waterhouse

SYN.: Lepus texianus Waterhouse

HASSTILESIIDAE

Hasstilesia tricolor (TX)

Sylvilagus aquaticus (Bachman) Swamp rabbit HASSTILESIIDAE

Hasstilesia tricolor (LA)

SCHISTOSOMATIDAE

Heterobilharzia americana (LA)

Sylvilagus aquaticus aquaticus (Bachman)

HASSTILESIIDAE

Hasstilesia tricolor (MS,TN)

Sylvilagus floridanus Allen Eastern cottontail FASCIOLIDAE

Fasciola hepatica (TX)

HASSTILESIIDAE

H. tricolor

(AL,AR, KS, KY,MD,MS,NC,PA,SC,VA)

Sylvilagus floridanus alacer (Bangs)

HASSTILESIIDAE

Hasstilesia tricolor (TX)

Sylvilagus floridanus mallurus (Thomas)

HASSTILESIIDAE

Hasstilesia tricolor (NC,NY)

Sylvilagus floridanus mearnsii (Allen)

HASSTILESIIDAE

Hasstilesia tricolor (IA,MI,MN,NY,WI)

Sylvilagus nuttallii (Bachman)Nuttall's cottontail

SYN.: Lepus sylvaticus Bachman

HASSTILESIIDAE

Hasstilesia tricolor (DC,PA,VA)

Sylvilagus palustris Bachman Marsh rabbit

HASSTILESIIDAE

H. tricolor (FL,NC)
NOTOCOTYLIDAE

Nudacotyle novicia (NC)

Sylvilagus palustris palustris (Bachman)

HASSTILESIIDAE

Hasstilesia tricolor (GA)

Sylvilagus transitionalis (Bangs) New England cottontail

HASSTILESIIDAE

Hasstilesia tricolor (CT)

\section{UNIDENTIFIED LEPORIDAE}

Cottontail

HASSTILESIIDAE

Hasstilesia tricolor

(AL,CT,KS,MD,MO,NJ,NY,PA,RI,VA,WV)

Hasstilesia spp. (NY)

Jack rabbit

HASSTILESIIDAE

Hasstilesia tricolor (TX)

Rabbit

FASCIOLIDAE

Fasciola hepatica (LA)

HASSTILESIIDAE

Hasstilesia tricolor (DC,MD,MS,NY,VA)

SCHISTOSOMATIDAE

Heterobilharzia americana (LA)

Varying hare

HASSTILESIIDAE

Hasstilesia tricolor (NY)

\section{FAMILY OCHOTONIDAE}

Ochotona collaris (Nelson)

Collared pika

BRACHYLAEMIDAE

Brachylaima spp. (AK)

\section{ORDER PINNIPEDIA}

FAMILY OTARIIDAE

Callorhinus ursinus (Linnaeus)

HETEROPHYIDAE

Northern

Cryptocotyle jejuna (AK)

Phocitrema fusiforme (AK)

Pricetrema zalophi(AK)

Eumetopias jubatus (Schreber) Northern sea lion

CAMPULIDAE

Zalophotrema hepaticum (OR)

HETEROPHYIDAE

Pricetrema eumetopii (AK)

P. zalophi (OR) 
Zalophus californianus (Lesson) CAMPULIDAE sea lion

Zalophotrema hepaticum (CA,NY,OR)

Zalophotrema spp. (CA)

HETEROPHYIDAE

Pricetrema zalophi (OR)

NANOPHYETIDAE

Nanophyetus salmincola (OR)

UNIDENTIFIED DIGENEA

Digenea gen. spp. (unspecified)

\section{FAMILY PHOCIDAE}

Erignathus barbatus (Erxleben) Bearded seal CAMPULIDAE

Orthosplanchnus fraterculus (AK)

Campulidae gen. spp. (AK)

HETEROPHYIDAE

Pricetrema spp. (AK)

Mirounga angustirostris (Gill)

HETEROPHYIDAE

Pricetrema zalophi (OR)

Phoca hispida Schreber

CAMPULIDAE

Orthosplanchnus arcticus (NWT)

Phoca vitulina Linnaeus

Harbor seal

HETEROPHYIDAE

Apophallus venustus (GA)

Cotylophallus similis (DC)

Cryptocotyle lingua (DC)

Pricetrema zalophi (OR)

Phoca vitulina largha Pallas

SYN.: Phoca largha Pallas

HETEROPHYIDAE

Phocitrema fusiforme (AK)

Phoca vitulina richardsii (Gray)

HETEROPHYIDAE

Pricetrema phocae (AK)

\section{ORDER RODENTIA}

FAMILY CAPROMYIDAE

Myocastor coypus (Molina)
SCHISTOSOMATIDAE
Heterobilharzia americana (LA)
Myocastor coypus bonariensis (Geoffroy Saint
Hilaire)
ECHINOSTOMATIDAE
Echinostoma revolutum (LA)
SCHISTOSOMATIDAE
Heterobilharzia spp. (LA)
Schistosomatidae gen. spp. (LA)

Northern

elephant seal
FAMILY CASTORIDAE

Castor canadensis Kuhl

ECHINOSTOMATIDAE

Echinostoma revolutum (NS)

FASCIOLIDAE

Fasciola hepatica (WA)

PARAMPHISTOMIDAE

Stichorchis subtriquetrus

(AK,Alta,CO,MN,Nfld,NS,Ont,OR,Que,TX)

PSILOSTOMIDAE

Stephanoproraoides lawi (MN,NS,Ont)

SCHISTOSOMATIDAE

Heterobilharzia americana (TX)

Castor canadensis carolinensis Rhoads

PARAMPHISTOMIDAE

Stichorchis subtriquetrus (LA)

Castor canadensis leucodontus Gray

PARAMPHISTOMIDAE

Stichorchis subtriquetrus (BC)

Castor canadensis mexicanus Bailey

PARAMPHISTOMIDAE

Stichorchis subtriquetrus (Mexico)

FAMILY ERETHIZONTIDAE

Erethizon dorsatum (Linnaeus) Porcupine

SCHISTOSOMATIDAE

Schistosomatium douthitti (Ont)

\section{FAMILY GEOMYIDAE}

Thomomys talpoides (Richardson) Northern NOTOCOTYLIDAE pocket gopher Quinqueserialis hassalli (WY)

\section{FAMILY HETEROMYIDAE}

Liomys pictus pictus (Thomas) Painted spiny BRACHYLAEMIDAE pocket mouse

Brachylaema bravoae (Mexico)

FAMILY MURIDAE

Clethrionomys gapperi (Vigors) Gapper's redPLAGIORCHIIDAE backed vole

Plagiorchis spp. (Nfld,Que)

Clethrionomys rutilus dawsoni (Merriam)

PLAGIORCHIIDAE Northern red-

Plagiorchis spp. (AK) backed mouse

Dicrostonyx groenlandicus rubricatus

(Richardson)

BRACHYLAEMIDAE Collared lemming

Brachylaima rauschi (AK) 
Microtus longicaudus baileyi Goldman

BRACHYLAEMIDAE Long-tailed vole

Scaphiostomum microti (CO)

Microtus montanus fusus Hall Montane vole NOTOCOTYLIDAE

Quinqueserialis hassalli (CO)

Microtus montanus nanus (Merriam)

SYN.: Microtus montanus caryi Bailey

NOTOCOTYLIDAE

Quinqueserialis hassalli (WY)

Microtus miurus muriei Nelson Singing vole

SYN.: Microtus miurus paneaki Rausch

BRACHYLAEMIDAE

Brachylaima rauschi (AK)

Microtus oeconomus macfarlani Merriam

NOTOCOTYLIDAE Tundra vole

Quinqueserialis quinqueserialis (AK)

Microtus pennsylvanicus (Ord) Meadow vole NOTOCOTYLIDAE

Quinqueserialis quinqueserialia

(Nfld,NY,Que)

PLAGIORCHIIDAE

Plagiorchis muris (Nfld,Que)

SCHISTOSOMATIDAE

Schistosomatium douthitti (Man, $\mathrm{OH}$ )

Microtus pennsylvanicus drummondii (Audubon

and Bachman)

NOTOCOTYLIDAE

Quinqueserialis quinqueserialis (AK)

Microtus pennsylvanicus modestus (Baird)

NOTOCOTYLIDAE

Quinqueserialis hassalli (CO,NM,WY)

Microtus pennsylvanicus pennsylvanicus (Ord)

BRACHYLAEMIDAE

Brachylaima thompsoni (MI,WI)

NOTOCOTYLIDAE

Notocotylus hassalli (MD,VA)

Nudacotyle novicia (IA)

Quinqueserialis hassalli (MD,MN,VA,WI)

PROSTHOGONIMIDAE

Mediogonimus ovilacus (MI)

SCHISTOSOMATIDAE

Schistosomatium douthitti (MI)

Microtus pennsylvanicus terraenovae Bangs

BRACHYLAEMIDAE

Brachylaima spp. (Nfld)

Mus musculus Rutty

House mouse

BRACHYLAEMIDAE

Brachylaema peromysci (KY)

DIPLOSTOMIDAE

Alaria americana (Ont)

A. arisaemoides (Ont)
Neofiber alleni nigrescens Howell RoundNOTOCOTYLIDAE tailed muskrat Quinqueserialis floridensis (FL)

Ondatra zibethicus (Linnaeus) Muskrat ALLASSOGONOPORIDAE

Allassogonoporus marginalis (MI)

DIPLOSTOMIDAE

Alaria craterum (Ont)

A. mustelae (AK,Man,Ont)

Fibricola cratera (NE)

ECHINOSTOMATIDAE

Echinochasmus schwartzi (MD,Ont,TX)

Echinoparyphium contiguum

(BC,KS,MI,NE,Ont)

Echinoparyphium spp. (NB)

Echinostoma callawayensis (AK,NE,Ont)

E. echinatum (PA)

$E$. revolutum

(BC,IL,KS,MA,Man,MD,MI,NB,NE,OH,Ont, OR,PA,Que)

Echinostoma spp. (Alta)

Echinostomatidae gen. spp. (NY)

HETEROPHYIDAE

Euryhelmis pacificus (OR)

Phagicola diminuta (TX)

LEUCOCHLORIDIOMORPHIDAE

Ptyalincola ondatrae (MI)

NOTOCOTYLIDAE

Catatropis fimbriata (NE)

Monostomum affinis (PA)

Notocotylus filamentis (AK,IL,Man,MI,Ont)

N. urbanensis (BC,MI,NB,Ont,PA,)

Notocotylus spp. (OR)

Nudacotyle novicia (IA,MI,MN,NB,Ont,TX)

Paramonostomum pseudalveatum (MD)

Quinqueserialis quinqueserialis

(AK,BC,IA,IL,Man,MD,MI,MT,NB,NE,OH,O

nt,OR,PA)

Q. zibethicus (Que)

OPISTHORCHIIDAE

Metorchis conjunctus (PA)

Opisthorchis tonkae (MN,NB)

Parametorchis spp.(MD)

PARAGONIMIDAE

Paragonimus kellicotti (KY)

Paragonimus spp. (MI)

PARAMPHISTOMIDAE

Stichorchis subtriquetrum (PA)

Wardius zibethicus

(Man,MI,NB,NE,OH,Ont,PA)

PLAGIORCHIIDAE

Plagiorchis noblei (Man)

P. proximus

(AK,BC,MA, MI,NB,NE,OH,Ont,PA)

PSILOSTOMIDAE 
Psilostomum ondatrae (Ont)

SCHISTOSOMATIDAE

Schistosomatium douthitti (MI,Man, MN,NB)

UROTREMATIDAE

Urotrema shillingeri (MD)

Ondatra zibethicus macrodon (Linnaeus)

ECHINOSTOMATIDAE

Echinochasmus schwartzi (MD)

NOTOCOTYLIDAE

Nudacotyle novicia (MD)

UROTREMATIDAE

Urotrema shillingeri (MD)

Ondatra zibethicus obscurus (Bangs)

DIPLOSTOMIDAE

Diplostomum mergi (Nfld)

ECHINOSTOMATIDAE

Echinoparyphium contiguum (Nfld)

E. recurvatum (Nfld)

Echinostoma revolutum (Nfld)

NOTOCOTYLIDAE

Quinqueserialis quinqueserialis (Nfld)

PARAMPHISTOMIDAE

Paramphistomidae gen. spp. (Nfld)

PLAGIORCHIIDAE

Palgiorchis proximus (Ont)

Ondatra zibethicus occipitalis (Elliot)

ECHINOSTOMATIDAE

Echinostoma revolutum (OR)

NOTOCOTYLIDAE

Notocotylus urbanensis (OR)

Ondatra zibethicus osoyoosensis (Lord)

DIPLOSTOMIDAE

Fibricola cratera (UT)

ECHINOSTOMATIDAE

Echinostoma revolutum (UT)

Echinostoma spp. (CO)

NOTOCOTYLIDAE

Notocotylus urbanensis (UT)

Paramonostomum pseudalveatum (UT)

Quinqueserialis quinqueserialis (CO,UT)

PLAGIORCHIIDAE

Plagiorchis proximus (CO,UT)

PSILOSTOMIDAE

Psilotrema spp. (UT)

Ondatra zibethicus rivalicius Bangs

SYN.: Ondatra rivalicia Bangs

ECHINOSTOMATIDAE

Echinochasmus schwartzi (LA)

HETEROPHYIDAE

Phagicola nana (LA)

NOTOCOTYLIDAE

Nudacotyle novicia (LA)

Paramonostomum pseudalveatum (LA)

Ondatra zibethicus zibethicus

OPISTHORCHIIDAE

Amphimerus pseudofelineus (IL)

Opisthorchis tonkae (NY)
PARAMPHISTOMIDAE

Wardius zibethicus (IL,MA,MD,NY,OH)

PLAGIORCHIIDAE

Plagiorchis proximus (IL,MA,NY,OH,WI)

SCHISTOSOMATIDAE

Schistosomatium douthitti (IL,OH)

Ondatra zibethicus zibethicus (Linnaeus)

DIPLOSTOMIDAE

Fibricola cratera (IL,OH)

ECHINOSTOMATIDAE

Echinoparyphium recurvatum (IL,ME,OH)

Echinostoma revolutum

(IL,MD,ME,NY,OH,WI)

NOTOCOTYLIDAE

Notocotylus filamentus (ME)

$N$. urbanensis $(\mathrm{NY}, \mathrm{OH})$

Notocotylus spp. (IL)

Nudacotyle novicia (IL, ME, $\mathrm{OH}$ )

Quinqueserialis quinqueserialis

(IL,MD, ME,NY,OH,WI)

OPISTHORCHIIDAE

Amphimerus pseudofelineus (IL)

Opisthorchis tonkae (NY)

PARAMPHISTOMIDAE

Wardius zibethicus (IL,MD,ME,NY,OH)

PLAGIORCHIIDAE

Plagiorchis proximus (IL, ME,NY,OH,WI)

PROSTHOGONIMIDAE

Mediogonimus ovilacus $(\mathrm{OH})$

SCHISTOSOMATIDAE

Schistosomatium douthitti (IL,OH)

Oryzomys palustris (Harlan) Marsh rice rat

DICROCOELIIDAE

Lyperosomum intermedium (FL,GA)

Zonorchis komareki (LA)

NOTOCOTYLIDAE

Catatrophis johnstoni (FL)

Peromyscus banderanus banderanus Allen

DICROCOELIIDAE Michoacan deer mouse

Dictyonograptus chamelensis (Mexico)

Peromyscus gossypinus gossypinus (Le Conte)

BRACHYLAEMIDAE

Scaphiostomum pancreaticum (GA)

DICROCOELIIDAE

Eurytrema komareki(GA)

Peromyscus guatemalensis guatemalensis

Merriam

BRACHYLAEMIDAE Guatemalan

Brachylaima chiapensis (Mexico) deer mouse

Peromyscus leucopus (Rafinesque)

BRACHYLAEMIDAE White-footed mouse

Brachylaima peromysci (KY,MD) 
B. virginiana $(\mathrm{NC})$

HETEROPHYIDAE

Euryhelmis squamula (WV)

SCHISTOSOMATIDAE

Schistosomatium douthitti (MI)

Peromyscus leucopus leucopus (Rafinesque)

BRACHYLAEMIDAE

Brachylaima peromysci (VA)

B. thompsoni (NC)

DIPLOSTOMIDAE

Alaria mustelae (MI)

Peromyscus leucopus noveboracensis (Fischer)

BRACHYLAEMIDAE

Brachylaima thompsoni (MI)

B. virginiana (MI)

DIPLOSTOMIDAE

Alaria mustelae (MI)

Peromyscus maniculatus (Wagner)

BRACHYLAEMIDAE

Deer mouse

Postharmostomum helicis (Que)

DIPLOSTOMIDAE

Alaria americana (Ont)

A. arisaemoides (Ont)

UNIDENTIFIED DIGENEA

Digenea gen. spp. (Que)

Peromyscus maniculatus bairdii (Hoy and

Kennicott)

DIPLOSTOMIDAE

Fibricola cratera (MI,WI)

Peromyscus maniculatus sonoriensis (Le Conte)

BRACHYLAEMIDAE

Scaphiostomum microti (UT)

Rattus norvegicus (Berkenhout) Norway rat

NANOPHYETIDAE

Nanophyetus salmincola(OR)

Rattus spp.

HETEROPHYIDAE

Phagicola diminuta (NY)

Reithrodontomys humulis

(Audubon and Bachman)

Eastern harvest

mouse

DICROCOELIIDAE

Zonorchis komareki (GA)

Sigmodon hispidus Say and Ord

OPISTHORCHIIDAE

Hispid

Metorchis conjunctus (Que)

PLAGIORCHIIDAE

Plagiorchis muris (TX)

\section{FAMILY SCIURIDAE}

Glaucomys sabrinus (Shaw)

PLAGIORCHIIDAE

Plagiorchis proximus (Sask)
Marmota monax (Linnaeus)

Woodchuck

NOTOCOTYLIDAE

Quinqueserialis hassalli (WI)

Quinqueserialis spp. (WI)

Marmota monax rufescens Howell

DICROCOELIIDAE

Dicrocoelium dendriticum (NY)

Sciurus carolinensis Gmelin

Gray squirrel

BRACHYLAEMIDAE

Brachylaima spp. (MD,NC)

NOTOCOTYLIDAE

Nudacotyle novicia (AL)

Sciurus carolinensis carolinensis Gmelin

NOTOCOTYLIDAE

Nudacotyle spp. (TN)

Spermophilus franklinii (Sabine) Franklin's

DIPLOSTOMIDAE

Alaria mustelae (Sask)

ground squirrel

PLAGIORCHIIDAE

Plagiorchis proximus (Sask)

Spermophilus richardsonii (Sabine) Richardson's PLAGIORCHIIDAE ground squirrel Plagiorchis proximus (Man,Sask)

Spermophilus variegatus utah Merriam

BRACHYLAEMIDAE Rock squirrel

Scaphiostomum microti (UT)

Tamias striatus (Linnaeus) Eastern chipmunk BRACHYLAEMIDAE

Scaphiostomum pancreaticum (Ont,WI)

Tamias striatus lysteri (Richardson)

BRACHYLAEMIDAE

Postharmostomum helicis (MI)

$P$. noveboracensis (NY)

Scaphiostomum pancreaticum (MI)

Tamiasciurus hudsonicus (Erxleben)

DIPLOSTOMIDAE

Red squirrel

Fibricola cratera (MI)

PLAGIORCHIIDAE

Plagiorchis proximus (Sask)

Tamiasciurus hudsonicus loquax (Bangs)

DIPLOSTOMIDAE

Fibricola cratera (MI)

\section{FAMILY ZAPODIDAE}

Zapus hudsonius (Zimmermann) Meadow NOTOCOTYLIDAE jumping mouse Quinqueserialis quinqueserialis (Nfld,Que) PLAGIORCHIIDAE

Plagiorchis proximus (Nfld,Que) 
SCHISTOSOMATIDAE

Schistosomatium douthitti (MI)

Zapus hudsonius hudsonius (Zimmermann)

NOTOCOTYLIDAE

Notocotylus hassalli (MI)

\section{ORDER SIRENIA \\ FAMILY TRICHECHIDAE}

Trichechus manatus Linnaeus Caribbean manatee SYN.: Manatus latirostris Harlan

PARAMPHISTOMIDAE

Chiorchis fabaceus (FL,PA)

\section{INFRACLASS METATHERIA ORDER MARSUPIALIA FAMILY DIDELPHIDAE}

Caluromys derbianus pallidus (Thomas)

SYN.: Philander laniger Wooly opossum pallidus Thomas

DICROCOELIIDAE

Eurytrema allentoshi (Panama)

OPISTHORCHIIDAE

Opisthorchis pricei (Panama)

Didelphis marsupialis Allen Southern opossum BRACHYLAEMIDAE

Brachylaima virginiana (GA)

PARAGONIMIDAE

Paragonimus kellicotti (GA,NC)

$P$. mexicanus (Costa Rica, Mexico)

$P$. rudis (Panama)

Paragonimus spp. (Mexico)

RHOPALIASIDAE

Rhopalias baculifer (FL)

$R$. coronatus (FL)

$R$. macracanthus (Mexico)

Didelphis marsupialis caucae Allen

SYN.: Didelphis marsupialis etensis Allen

RHOPALIASIDAE

Rhopalias coronatus (Costa Rica)

$R$. horridus (Costa Rica)

Didelphis marsupialis tabascensis Allen

SYN.: Didelphis mesamericana tabascensis

Allen

RHOPALIASIDAE

Rhopalias coronatus (Mexico)

R. macracanthus (Mexico)

Didelphis virginiana Kerr

SYN.: Didelphis mesamericana mesamericana

Oken

BRACHYLAEMIDAE
Brachylaima didelphus (FL)

B. opisthotrias (IL,PA,TN,TX,VA)

B. o. virginiana (VA)

B. virginiana (GA,LA,NC,TN)

CYATHOCOTYLIDAE

Linstowiella szidati (LA)

DICROCOELIIDAE

Eurytrema allentoshi (TX)

DIPLOSTOMIDAE

Didelphodiplostomum variabile

(FL,IL,NC,TN,TX)

Fibricola cratera (FL,GA,TN)

F. lucida (FL,GA,LA,TN,TX)

ECHINOSTOMATIDAE

Echinostoma revolutum (IL)

Echinostoma spp. (NC)

HETEROPHYIDAE

Phagicola diminuta (FL)

MICROPHALLIDAE

Maritreminoides nettae (NC)

OPISTHORCHIIDAE

Amphimerus pseudofelineus (IL)

PARAGONIMIDAE

Paragonimus rudis (Guatamala)

P. westermani (TN)

RHOPALIASIDAE

Rhopalias coronatus

(Guatemala,Mexico,TX,VA)

R. horridus (Guatamala)

R. macracanthus

(FL,GA,IL,LA,NC,OK,PA,TN,TX)

SCHISTOSOMATIDAE

Heterobilharzia americana (LA)

Didelphis virginiana californica Bennett

SYN.: Didelphis marsupialis mesamericana

Oken

PARAGONIMIDAE

Paragonimus mexicanus (Mexico)

RHOPALIASIDAE

Rhopalias macracanthus (Mexico)

Didelphis virginiana virginiana Kerr

SYN.: Didelphis marsupialis virginiana

(Kerr)

BRACHYLAEMIDAE

Brachylaima virginiana (MI,VA)

DIPLOSTOMIDAE

Fibricola cratera (TX)

ECHINOSTOMATIDAE

Echinostoma revolutum (MI)

Didelphis spp.

BRACHYLAEMIDAE

Brachylaima opisthotrias (IL)

B. virginiana (CA, IL, VA)

DIPLOSTOMIDAE

Didelphodiplostomum variabile (GA)

Fibricola lucida (IL)

RHOPALIASIDAE 
Rhopalias macracanthus (IL)

Marmosa mexicana mexicana Merriam RHOPALIASIDAE Mexican mouse-opossum Rhopalias coronatus (Guatamala)

Metachirops opossum Miller Gray four-eyed SYN.: Philander opossum Miller opossum PARAGONIMIDAE Paragonimus rudis (Panama)

Metachirops opossum fuscogriseus Allen SYN.: Philander opossum fuscogriseus (Allen) MICROPHALLIDAE Maritrema prolixum (Costa Rica)
OPISTHORCHIIDAE

Amphimerus neotropicalis (Costa Rica) PARAGONIMIDAE

Paragonimus mexicanus (Costa Rica)

$P$. rudis (Costa Rica) RHOPALIASIDAE

Rhopalias baculifer (Costa Rica)

$R$. coronatus (Costa Rica)

$R$. horridus_(Costa Rica)

Metachirops opossum pallidus (Allen)

SYN.: Philander opossum pallidus (Allen)

PARAGONIMIDAE

Paragonimus mexicanus (Mexico) 


\section{REFERENCES}

ABRAM, J. B. 1969. Some gastrointestinal helminths of Ondatra zibethicus Linnaeus, the muskrat in Maryland. Proceedings of the Helminthological Society of Washington 36: 93-95.

ADDISON, E. M., AND B. BOLES. 1978. Helminth parasites of wolverine, Gulo gulo, from the District of MacKenzie, Northwest Territories. Canadian Journal of Zoology 56: 2241-2242.

ADDISON, E. M., M. J. PYBUS, AND H. J. RIETVELD. 1978. Helminths and arthropod parasites of black bear, Ursus americanus in central Ontario. Canadian Journal of Zoology 56: 2122-2126.

AITON, J. F. 1938. Enlarged spleen in whitetail deer at Glacier National Park.

Transactions of the Third North American Wildlife Conference 3: 890-892.

ALEXANDER, A. D., V. FLYGER, Y. F. HERMAN, S. J. MCCONNELL, N. ROTHSTEIN, AND R. H. YAGER. 1972. Survey of wild mammals in a Chesapeake Bay area for selected zoonoses. Journal of Wildlife Diseases 8: 119-126.

ALICATA, J. E. 1932. A new trematode, Acanthatrium eptesici, from the brown bat. Journal of the Washington Academy of Sciences 22: 271-274.

AMEEL, D. J. 1931. More data on the lung fluke, Paragonimus, in North America. Science 74: 493-494.

1932. The muskrat, a new host for Paragonimus. Science 75: 382.

-- 1934. Paragonimus, its life history and distribution in North American and its taxonomy (Trematoda: Troglotrematidae). American Journal of Hygiene 19: 279-317. 1938. The morphology and life cycle of Euryhelmis monorchis n. sp. (Trematoda) from the mink. Journal of Parasitology 24: 219-224.

-1942. Two larval cestodes from the muskrat. Transactions of the American Microscopical Society 61: 267-271. -- 1944. The life history of Nudacotyle novicia Barker, 1916 (Trematoda:

Notocotylidae). Journal of Parasitology 30: 257-263.

ANDERSON, D. R., AND R. L. BEAUDOIN. 1966. Host habitat and age as factors in the prevalence of intestinal parasites of the muskrat. Bulletin of the Wildlife Disease Association 2: 70-77.

ANDREWS, C. L., W. R. DAVIDSON, AND E. E PROVOST. 1980. Endoparasites of selected populations of cottontail rabbits (Sylvilagus floridanus ) in the southeastern United States. Journal of Wildlife Diseases 16: 395-401.

ANONYMOUS, 1930. Experimental fur farm. Ontario Department of Game Fish Annual Report 23 (1929) 9-12.

-.--1931. A note on Parametorchis canadensis (Law) from Ontario mink. Report of the Ontario Veterinary College for 1930: 39 .

1950. Survey to ascertain the geographical distribution of the large American liver fluke ( Fascioloides magna) in Ontario. Report of the Ontario Veterinary College for 1949: 35.

ARATA, A. A. 1959. Ecology of muskrats in stripmine ponds in southern Illinois. Journal of Wildlife Management 23: 177-186.

ARCHER, J., S. J. TAFT, AND R. P. THIEL. 1986. Parasites of wolves, Canis lupus, in Wisconsin, as determined from fecal examinations. Proceedings of the Helminthological Society of Washington 53: 290-291.

AUGUSTINE, D. L., AND C. URIBE. 1927. Alaria arisaemoides, n.sp., a trematode from Vulpes fulva. Parasitology 19: 236244.

BABERO, B. B. 1953 . Some helminth parasites of Alaskan beavers. Journal of Parasitology 39: 674-675. -1957. Some helminths from Illinois opossums. Journal of Parasitology 43: 232. -- 1960a. Further studies on helminths of the opossum, Didelphis virginiana, with a description of a new species from this host. Journal of Parasitology 46: 455-463. 1960b. A survey of parasitism in skunks (Mephitis mephitis) in Louisiana, with observations on pathological damages due to helminthiasis. Journal of Parasitology 46 (section 2, supplementary): 26-27. 1972. A record of progenesis in trematoda. Proceedings of the Helminthological Society of Washington, 39(1): 128-136. 
BABERO, B. B., L. H. KARSTAD, AND J. R. SHEPPERSON. 1959. Studies on helminth parasitism of feral swine in Georgia. Bulletin of the Georgia Academy of Science 17: 94-104.

BABERO, B. B., AND J. W. LEE. 1961. Studies on the helminths of nutria, Myocastor coypus (Molina), in Louisiana with checklist of other worm parasites from this host. Journal of Parasitology 47: 378-390.

BABERO, B. B., AND R. RAUSCH. 1952. Notes on some trematodes parasitic in Alaskan Canidae. Proceedings of the Helminthological Society of Washington 19: 15-17.

BABERO, B. B., AND J. R. SHEPPERSON. 1958. Some helminths of raccoons in Georgia. Journal of Parasitology 44: 519.

BAFUNDO, K. W., W. E. WILHELM, AND M. L. KENNEDY. 1980. Geographic variation in helminth parasites from the digestive tract of Tennessee raccoons, Procyon lotor. Journal of Parasitology 66: 134-139.

BALL, L. 1952. Notes on helminth parasites of muskrats from western Colorado. Journal of Parasitology 38: 83-84.

BARKER, F. D. 1915. Parasites of the American muskrat (Fiber zibethicus ). Journal of Parasitology 1: 184-197.

- 1916. A new monostome trematode parasitic in the muskrat with a key to the parasites of the American muskrat. Transactions of the American Microscopical Society 35: 175-184.

BARKER, F. D., AND J. W. LAUGHLIN. 1911. A new species of trematode from the muskrat, Fiber zibethicus. Transactions of the American Microscopical Society 30: 261274.

BARON, R. W. 1970. The occurrence of Echinococcus multilocularis Leuckart, 1863 and of other helminths in the red fox, Vulpes vulpes, in southern Manitoba. Canadian Journal of Zoology 48: 1132.

BEAVER, P. C. 1941. Studies on the life history of Euparyphium melis (Trematoda:

Echinostomidae). Journal of Parasitology 27: 35-44.

BECKERDITE, F. W., G. C. MILLER, AND R. HARKEMA. 1971. Observations on the life cycle of Pharyngostomoides spp. and the description of $P$. adenocephala sp. $\mathrm{n}$. (Strigeoidea: Diplostomatidae) from the raccoon, Procyon lotor (L.). Proceedings of the Helminthological Society of Washington 38: 149-156.
BECKETT, J. V., AND V. GALLICCHIO. 1966. Occurrence of the lung fluke, Paragonimus kellicotti Ward, 1908, in Ohio mink. Journal of Parasitology 52: 511.

1967. A survey of helminths of the muskrat, Ondatra z. zibethica Miller, 1912, in Portage County, Ohio. Journal of Parasitology 53: 1169-1172.

BEHREND, D. F., G. F. MATTFELD, AND J. E. WILEY III. 1973. Incidence of liver flukes in a sample of white-tailed deer from the Adirondacks. New York Fish and Game Journal 20: 158-161.

BELL, J. F., AND W. S. CHALGREN. 1943. Some wildlife diseases in the eastern United States. Journal of Wildlife Management 7: 270-278.

BEMRICH, W. J., AND J. C. SCHLOTTHAUER. 1971. Paragonimus kellicotti (Ward, 1908) in a Minnesota skunk (Mephitis mephitis). Journal of Wildlife Diseases 7: 36.

BENNETT, H. J., AND A. G. HUMES. 1939. Studies on the pre-cercarial development of Stichorchis subtriquetrus (Trematoda: Paramphistomidae). Journal of Parasitology 25: 223-229.

BERGSTROM, R. C. 1967. Sheep liver fluke, Fasciola hepatica L., 1758 from buffalo, Bison bison (L., 1758) in western Wyoming. Journal of Parasitology 53: 724.

BEVERLEY-BURTON, M., AND P. R. SWEENY. 1972. Intranuclear, paracrystalline inclusions in various cells of Quinqueserialis quinqueserialis and Notocotylus urbanensis (Trematoda: Notocotylidae). Canadian Journal of Zoology 50: 345-348.

BISHOP, L. 1979. Parasite-related lesions in a bearded seal, Erignathus barbatus. Journal of Wildlife Diseases 15: 285-293.

BLANKESPOOR, H. D., AND P. A. SZYMUSIAK. 1974. Helminths from Myotis keenii (Merriam) in eastern Iowa. Journal of Parasitology 60: 934.

BLANKESPOOR, H. D., AND M. J. ULMER. 1970. Helminths from six species of Iowa bats. Proceedings of the Iowa Academy of Science 77: 200-206. 1972. Prosthodendrium volaticum sp. n. (Trematoda: Lecithodendriidae) from two species of Iowa bats. Proceedings of the Helminthological Society of Washington 39: 224-226.

BOSMA, N. J. 1931. Alaria mustelae sp. nov., a trematode requiring four hosts. Science 74 : 521-522. 
1934. The life history of the trematode Alaria mustelae, Bosma, 1931.

Transactions of the American Microscopical Society 53: 116-153.

BRENES, R. R., R. ZELEDON, AND G. ROJAS. 1980. Biological cycle and taxonomic position of a Costa Rican Paragonimus and the present status of Paragonimus from the new world. Brenesia 18: 353-366.

BROOKS, D. R., AND J. R. COGGINS. 1983. Limatulum mcdanieli n. sp. (Digenea: Lecithodendriidae) from Myotis lucifugus (Mammalia: Chiroptera) in Wisconsin. Transactions of the American Microscopical Society 102: 81-83.

BROWN, D. H., R. W. McINTYRE, C. A. DELLI QUADRI, AND R. J. SCHROEDER. 1960. Health problems of captive dolphins and seals. Journal of the American Veterinary Medical Association 137: 534-538.

BROWNING, B. M., AND E. M. LAUPPE. 1964. A deer study in a Redwood-Douglas fir forest type. California Department of Fish and Game Game Bulletin 50: 132-147.

BUMP, G. 1937. Bureau of game. 26th Annual Report of the New York State Conservation Department 288-335.

1940. Bureau of game. 29th Annual Report of the New York State Conservation Department 230-270.

-- 1941. Bureau of game. 30th Annual Report of the New York State Conservation Department 213-260. 1942. Bureau of game. 31st Annual Report of the New York State Conservation Department 209-264.

BURNS, W. C., AND I. PRATT. 1953. The life cycle of Metagonimoides oregonensis Price (Trematoda: Heterophyidae). Journal of Parasitology 39: 60-69.

BUSH, A. O., AND J. M. KINSELLA. 1972. A natural definitive host for Catatropis johnstoni Martin, 1956 (Trematoda: Notocotylidae), with notes on experimental host specificity and intraspecific variation. Journal of Parasitology 58: 843-845.

BUSH, A. O., AND W. M. SAMUEL. 1981. A review of helminth communities in beaver (Castor spp.) with a survey of Castor canadensis in Alberta, Canada. In: Proceedings of the First Worldwide Furbearer Conference. J.A. Chapman and D. Pursley (editors). pp 678-689.

BUTLER, W. J. 1932. Report of the Montana livestock sanitary board. Report of the Montana Livestock Sanitary Board and State Veterinary Surgeon 1: 3-24.
BUTTERWORTH, E. W., AND J. C. HOLMES 1984. Character divergence in two species of trematodes (Pharyngostomoides: Strigeoidea). Journal of Parasitology 70 : 315-316.

BYRD, E. E., B. J. BOGITSH, AND W. P. MAPLES. 1961. Grysoma singularis, a new species of trematodes (Digenea: Psilostomidae) from the racoon Procyon lotor (L.). Journal of Parasitology 47: 783786.

BYRD, E. E., AND R. W. MACY. 1942.

Mammalian trematodes. III. Certain species from bats. Journal of the Tennessee Academy of Science 17(1): 149-156.

BYRD, E. E., A. K. PRESTWOOD AND W. P. MAPLES. 1967. A new host and two new locality records for the blood fluke, Heterobilharzia americana Price, 1929. Journal of Parasitology 53: 1115-1116.

BYRD, E. E., AND R. J. REIBER. 1942a. Mammalian trematodes. I. Trematodes from the opossum, Didelphis virginiana Kerr. Journal of the Tennessee Academy of Science 17: 130-142.

1942b. Mammalian trematodes. II. Three flukes from small mammals. Journal of the Tennessee Academy of Science 17: 143-148.

BYRD, E. E., R. J. REIBER, AND M. V. PARKER. 1942. The anatomy of a lung fluke from the opossum (Didelphis virginiana Kerr). Journal of the Tennessee Academy of Science 17: 116-129.

CABALLERO Y C., E. 1940. Algunos trematodos intestinales de los murcielagos de Mexico I. Anales del Instituto de Biologia. Universidad de Mexico 11: 215-223.

1942. Trematodes de los murcielagos de Mexico. III.Descripcion de Urotrema scabridum Braun 1900. Y Posicion sistematica de las especies Norteamericanas de este genero. Sobretiro de Jose Anales del Instituto de Biologia 13: 642-648.

1943a. Trematodos de los murcielagos de Mexico. IV. Descripcion de un nuevo genero de la subfamilia Lecithodendriinae Looss, 1902, y una nueva especie de

Prosthodendrium Dollfus, 1931. Anales del Instituto de Biologia. Universidad de Mexico 14: 173-193.

- 1943b. Algunas especies de trematodos de los murcielagos de la region de Izucar de Matamoros, Pue. V. Anales del Instituto de Biologia. Universidad de Mexico 14: 423430 . 
1946. Estudios helmintologicos de la region oncocercosa de Mexico y de la Republica de Guatemala. Trematoda. II. Presencia de Paragonimus en reservorios naturales y descripcion de un Nuevo Genero. Anales del Instituto de Biologia. Universidad de Mexico 17: 137-165. - 1947. Stichorchis subtriquetrus (Rudolphi,1814) en los castores del estado de Nuevo Leon, Mexico. Anales del Instituto de Biologia. Universidad de Mexico 18: 165168.

1964a. Helmintos de la Republica de

Panama, XXIV. Descripcion de tres especies de trematodos Lecithodendriidae que parasitan al murcelago Myotis nigricans nigricans (Schinz). Anales De La Escuela Nacional De Ciencias Biologicas 13: 73-82. 1964b. Tres nuevas especies de trematoda Rudolphi, 1808 que parasitan a murcielagos (Chiroptera Blumenbach, 1774) de America Latina. Universidad de Nuevo Leon Mexico 5: 1-34.

CABALLERO, Y C. E., M. BRAVO-HOLLIS, Y M. C. CERECORO. 1944. Estudios helmintologicos de la region oncocercosa de Mexico y de la Republica de Guatemala Trematoda I. Anales del Instituto de Biologia. Universidad de Mexico 75: 59-72.

CABALLERO Y C. E., R. R. BRENES, AND O. JIMENEZ-QUIROS. 1957. Helmintos de la Republica de Costa Rica. IV. Algunos Trematodes de animales domesticos y silvestres. Revista De Biologia Tropical 5: 135-155.

CABALLERO, Y C. E., AND G. R. CABALLERO. 1969a. Un nuevo genero de trematodo digeneo de los conductos biliares de un mamifero de la Republica de Costa Rica, America Central. Anales de la Escuela Nacional De Ciencias Biologicas Mexico 18: 277-285.

-- 1969b. Trematodos de los murcielagos de Mexico. IX. Descripcion de una nueva especie de Platynosomum Looss, 1907 y a continuacion el primer supplemento del catalogo taxonomico de los trematodos que parasitan a los murcielagos. Revista De La Sociedad Mexicana De Historia Natural 30: 263-290.

CABALlERO, Y C. E., AND M. C. CERECERO. 1942. Trematodos de los murcielagos de Mexico. II. Redescripcion y posicion sistematica de Distomum tubiporum Braun, 1900. Anales del Instituto de Biologia. Universidad de Mexico 13: 97-104.
CABALLERO, Y C. E., AND F. M. GEI. 1961. Descripcion de dos trematodos de un marsupial de la Republica de Costa Rica y un catalogo de los trematodos que parasitan a Marsupialies Illiger, 1811. Anales De La Escuela Nacional De Ciencias Biologicas 10: 45-86.

\section{Presencia de Castroia silvai}

Travassos, 1928, (Trematoda Digenea) en un murcielago de Costa Rica. Anales de la Escuela Nacional De Ciencias Biologicas 12: 85-89.

CABALLERO Y C. E., F. M. GEI, AND G. R. CABALLERO. 1963. Una nueva especie de Opistorquido (Trematoda, Digenea) parasita del pancreas de un marsupial. Anales De La Escuela Nacional De Ciencias Biologicas 12: 79-84.

CABALLERO Y C., E., AND M. C. Y D. ZERECERO. 1951. Trematodos de los murcielagos de Mexico. VII. Presencia de Prosthodendrium macnabi Macy, 1936, en Lasiurus cinereus (Beauvois). Anales del Instituto de Biologia. Universidad de Mexico 22: 159-167.

CAIN, G. D. 1966. Helminth parasites of bats from Carlsbad Caverns, New Mexico. Journal of Parasitology 52: 351-357.

CAIN, G. D., AND E. H. STUDIER. 1972. Plagiorchis (Plagiorchis) micracanthos Macy, 1931 (Trematoda: Digenea), from Myotis lucifugus occultus in north-east New Mexico. Southwestern Naturalist 17: 104105.

1974. Parasitic helminths of bats from the southwestern United States and Mexico. Proceedings of the Helminthological Society of Washington 41: 113-114.

CAMERON, A. E. 1923. Notes of buffalo: anatomy, pathological conditions, and parasites. Veterinary Journal 79: 331-336.

CAMERON, T. W. M. 1936. Studies on the heterophyid trematode, Apophallus venustus (Ransom, 1920) in Canada. Part I. Morphology and taxonomy. Canadian Journal of Research Section D, Zoological Sciences 14: 59-69.

1937a. Studies on the heterophyid trematode Apophallus venustus (Ransom, 1920) in Canada. Part II. Life history and bionomics. Canadian Journal of Research Section D, Zoological Sciences 15: 38-51. - 1937b. Cited in: The distribution of Paragonimus. Journal of Parasitology 23: 382-388. 
CANAVAN, W. P. N. 1934. On a trematode Allopyge undulatus n.sp. parasitic in Lilford's crane (Megalornis grus lilfordi). Parasitology 26: 117-120.

CARMICHAEL, A. C., AND A. E. MUCHLINSKI. 1980. Survival of Schistosomatium douthitti during hibernation in the natural host, Zapus hudsonius. Journal of Parasitology 66: 365-366.

CHADDOCK, T. T. 1948. Some facts relative to disease as found in wildlife. North American Veterinarian 29: 560-567.

CHANDLER, A. C. 1929. A new species of trematode worms belonging to the genus Hasstilesia from rabbits in Texas.

Proceedings of theUnited States National Museum 75: 1-5.

1932. Notes on the helminth parasites of the opossum (Didelphus virginiana) in southeast Texas, with descriptions of four new species. Proceedings of the United States National Museum 81(16): 1-15. 1938. A report on the parasites of a bat, Nycticeius humeralis, with descriptions of four new helminths. In A. Neiva, ed. Livro Jubilar Professor Lauro Travassos. Instituto Oswaldo Cruz, Rio de Janeiro, Brazil. pp. 107-114.

1941. Helminths of muskrats in southeast Texas. Journal of Parasitology 27: 175-181.

1942a. The helminths of raccoons in east Texas. Journal of Parasitology 28: 255-268. -- 1942b. The morphology and life cycle of a new strigeid, Fibricola texensis, parasitic in raccoons. Transactions of the American Microscopical Society 61: 156-167.

- 1946. Helminths of armadillos, Dasypus novemcinctus, in eastern Texas. Journal of Parasitology 32: 237-241.

- 1954. New strigeids from Minnesota birds and mammals. American Midland Naturalist 52: 133-141

CHANDLER, A. C., AND R. RAUSCH. 1946. A study of strigeids from Michigan mammals, with comments on the classification of mammalian strigeids. Transactions of the American Microscopial Society 65: 328-337.

CHEATUM, E. L. 1942. Comparative disease studies (Supplement E). Pittman-Robertson Quarterly 2: 215.

-- 1951. Disease in relation to winter mortality of deer in New York. Journal of Wildlife Management 15: 216-220.

CHENG, T. C. 1957. Studies on the genus Acanthatrium Faust, 1919 (Trematoda: Lecithodendriidae); with the description of two new species. Journal of Parasitology 43: 60-65.

-- 1959. The description of Acanthatrium beuschleini n. sp., a new trematode parasite of bats; and a re-evaluation of the reproductive system of Acanthatrium sogandaresi Coil and Kuntz, 1958. Journal of Parasitology 45: 323-326.

CHING, H. L., AND E. S. ROBINSON. 1959. Two campulid trematodes from a new host, the harbor porpoise. Journal of Parasitology 45: 181.

CHOQUETTE, L. P. E., AND D. H. PIMLOTT. 1956. Gastrointestinal parasites of beaver in Newfoundland. Canadian Journal of Zoology 34: 209.

CHOQUETTE, L. P. E., E. BROUGHTON, AND G. G. GIBSON. 1973. Schistosomatium douthitti (Cort, 1914) Price, 1929 in a porcupine (Erethizon dorsatum) in eastern Ontario, Canada. Canadian Journal of Zoology 51: 1317.

CHOQUETTE, L. P. E., G. G. GIBSON, AND B. SIMARD. 1971. Fascioloides magna (Bassi, 1875) Ward, 1917 (Trematoda) in woodland caribou, Rangifer tarandus caribou (Gmelin), of northeastern Quebec, and its distribution in wild ungulates in Canada. Canadian Journal of Zoology 49: 280-281.

CHOQUETTE, L. P. E., G. G. GIBSON, E. KUYT, AND A. M. PEARSON. 1973.Helminths of wolves, Canis lupus L., in the Yukon and Northwest Territories. Canadian Journal of Zoology 51: 1087-1091.

CHRISTIAN, F. A. 1972. Acanthatrium umbraculatum $\mathrm{sp}$. $\mathrm{n}$.

(Digenea:Lecithodendriidae) from the little brown bat, Myotis lucifugus. Journal of Parasitology 58: 710-711.

CIORDIA, H. 1949. Cytological study of Rhopalias macracanthus Chandler, 1932, a trematode from the opossum, Didelphis virginiana. Journal of Parasitology 35: 417 422.

COGGINS, J. R., J. L. TEDESCO, AND C. E. RUPPRECHT. 1981. Intestinalhelminths of the bat, Myotis keenii (Merriam), from southeastern Wisconsin. Proceedings of the Helminthological Society of Washington 48 : 93-96. 1982. Seasonal changes and overwintering of parasites in the bat, Myotis lucifugus (Le Conte), in a Wisconsin hibernaculum. American Midland Naturalist 107: 305-315. 
CONKLIN, R. L., AND A. D. BAKER. 1930.

Presence of the lancet fluke,Dicrocoelium dendriticum (Rudolphi, 1819) in Canada. Journal of Parasitology 17: 18-19.

CONTI, J. A., D. J. FORRESTER, AND J. R. BRADY. 1983. Helminths of black bears in Florida. Proceedings of the Helminthological Society of Washington 50 : 252-256.

COOPER, A. R. 1921. Trematodes and cestodes of the Canadian Arctic Expedition. Report of the Canadian Arctic Expeditions, 19131918, \#19, Parts G-H: 1-27.

COWAN, D. F. 1966. Pathology of the pilot whale Globicephala melaena.A comparative survey. Archives of Pathology 82: 178-189. 1967. Helminth parasites of the pilot whale Globicephala melaena (Traill, 1809). Journal of Parasitiology 53: 166-167.

COWAN, I. MCT. 1942. Report upon some diseases and parasites of game birds and game and fur-bearing mammals in British Columbia. Report of the Provincial Game Commission, British Columbia (1941), K40K45.

1946. Parasites, diseases, injuries, and anomalies of the Columbian black-tailed deer, Odocoileus hemionus columbianus (Richardson), in British Columbia.

Canadian Journal of Research Section D, Zoological Sciences 24: 71-103.

1951. The diseases and parasites of big game animals of Western Canada.

Proceeding of the Fifth Annual Game Convention, Vancouver, B.C. 1951 5: 37-64.

CRAM, E. B. 1926. Wild carnivores as hosts of the trematode previously found in dogs as the result of salmon poisoning. The North American Veterinarian $7: 42-43$.

CUSTER, J. W., AND D. B. PENCE. 1981. Ecological analyses of helminth populations of wild canids from the gulf coastal prairies of Texas and Louisiana. Journal of Parasitology 67: 289-307.

DAHLBERG, B. L., AND R. C. GUETTINGER. 1956. The white-tailed deer in Wisconsin. Technical Wildlife Bulletin No. 14, Game Management Division, Wisconsin Conservation Department: $282 \mathrm{pp}$.

DAILEY, M. D. 1971. Distribution of helminths in the dall porpoise (Phocoenoides dalli True). Journal of Parasitology 57: 1348.

DAILEY, M. D., AND S. H. RIDGWAY. 1976. A trematode from the round window of an Atlantic bottlenosed dolphin's ear. Journal of Wildlife Diseases 12: 45-47.
DAILEY, M., AND R. STROUD. 1978. Parasites and associated pathology observed in cetaceans stranded along the Oregon coast. Journal of Wildlilfe Disease 14: 503-511.

DAILEY, M. D., AND W. A. WALKER. 1978. Parasitism as a factor (?) in single strandings of southern California cetaceans. Journal of Parasitology 64: 593-596.

DAVIDSON, W. R. 1976. Endoparasites of selected populations of gray squirrels (Sciurus carolinensis) in the southeastern United States. Proceedings of the Helminthological Society of Washington 43: 211-217.

DAVIDSON, W. R., J. L. BLUE, L. B. FLYNN, S. M. SHEA, R. L. MARCHINTON, AND J. A. LEWIS. 1987. Parasites, diseases and health status of sympatric populations of samber deer, and white-tailed deer in Florida. Journal of Wildlife Diseases 23: 267-272.

DELOYA, J. C. 1970. Descripcion de Brachylaemus (Brachylaemus) bravoae sp. nov. (Trematoda: Digenea), de roedores del estado de Jalisco, Mexico. Anale del Instituto de Biologia. Nal. Auton. Mexico. 41. Ser. Zoologia, Num Unico: 39-44.

DENTON, J. F. 1942. Eurytrema procyonis, n. sp. (Trematoda: Dicrocoeliidae), from the raccoon, Procyon lotor. Proceedings of the Helminthological Society of Washington 9: 29-30.

1944a. The occurrence of Eurytrema allentoshi (Foster, 1939) in the opossum in Texas. Proceedings of the Helminthological Society of Washington 11: 54-55.

- 1944b. Studies on the life history of Eurytrema procyonis Denton, 1942. Journal of Parasitology 30: 277-286.

DENTON, J. F., AND J. M. KINSELLA. 1972.

Lyperosomum intermedium sp. n. (Digenea: Dicrocoeliidae) from the rice rat, Oryzomys palustris , from southeastern salt marshes. Journal of Parasitology 58: 226-228.

DIBBLE, E. D., W. F. FONT, AND D. D. WITTROCK. 1983. Helminths of the red fox, Vulpes vulpes L., in West Central Wisconsin. Journal of Parasitology 69: 1170-1172.

DICK, T. A., AND R. D. LEONARD. 1979.

Helminth parasites of fisher Martes pennanti (Erxleben) from Manitoba, Canada. Journal of Wildlife Diseases 15: 409-412.

DICKERSON, L. M. 1930. A new variety of Harmostomum opisthotrias from the North American opossum, Didelphys virginiana, 
with a discussion of its possible bearing on the origin of its host. Parasitology 22: 3746.

DIKMANS, G. 1930. Fasciola hepatica from the bile ducts of a rabbit. Journal of Parasitology 16: 162.

DINABURG, A. G. 1939. Helminth parasites collected from deer Odocoileus virginianus, in Florida. Proceedings of the Helminthological Society of Washington 6 : 102-104.

DODDS, D. G., AND J. S. MACKIEWICZ. 1961. Some parasites and diseases of snowshoe hares in Newfoundland. Journal of Wildlife Management 25: 409-414.

DORNEY, R. S., AND L. H. LAUERMAN JR. 1969. A helminthological survey of wild mink in Wisconsin. Bulletin of the Wildlife Disease Association 5: 35-36.

DUBOIS, G. 1951. Etude des trematodes NordAmericains de la collection E. L. Schiller et revision du genre Notocotylus Diesing, 1839. Bulletin de la Societe Neuchateloise des Sciences Naturelles 74: 41-76.

DUBOIS, G., AND R. RAUSCH. 1950. Troisieme contribution a l'etude des Strigeides (Trematoda) Nord-Americains. Bulletin de la Societe Neuchateloise des Sciences Naturelles 73: 19-50.

1960. Quatrieme contribution a l'etude des Strigeides (Trematoda) NordAmericains. Bulletin de la Societe Neuchateloise des Sciences Naturelles 83: 79-92.

DUFF, D. 1914. The beaver fluke, Amphistomum subtriquetrum, Rudolphi. Transactions of the Royal Society of Canada 8 (series 3 , section 4): 87-89.

DUNAGAN, T. T. 1957a. Studies on the parasites of edible animals of Alaska. Alaskan Air Command Arctic Aeromedical Laboratory, Ladd Air Force Base. Technical Note AALTN-57-14. Report on Project No. 7955-4. 1957b. Helminth parasites of Alaskan muskrats. Transactions of the American Microscopical Society 76: 318-320.

DUNN, A. M. 1968. The wild ruminant as reservoir host of helminth infection. Symposia of the Zoological Society of London 24: 221-248.

DUTSON, V. J., J. N. SHAW, AND S. E. KNAPP. 1967. Epizootiologic factors of Fascioloides magna (Trematoda) in Oregon and southern Washington. American Journal of Veterinary Research 28: 853-860.
DYER, W. G. 1970. Helminths of the striped skunk, Mephitis mephitis Schreber, in North Dakota. Proceedings of the Helminthological Society of Washington 37: 92-93.

DYER, W. G., AND W. D. KLIMSTRA. 1977. Gastrointestinal helminths of the muskrat, Ondatra zibethicus, in southern Illinois. Transactions of the I11inois State Academy of Science 70: 356-362.

ECKERLIN, R. P. 1973. Hasstilesia tricolor (Stiles and Hassall, 1894) Hall, 1916, from a new host, Sylvilagus transitionalis in Connecticut. Journal of Parasitology 59: 1137-1138.

EDWARDS, R. L. 1949. Internal parasites of central New York muskrats ( Ondatra $z$. zibethica L.). Journal of Parasitology 35 : 547-548.

ERICKSON, A. B. 1938. Parasites of some Minnesota Cricetidae and Zapodidae, and a host catalogue of helminth parasites of native American mice. American Midland Naturalist 20: 575-589. 1940. Euparyphium melis (Trematoda: Echinostomidae) from the snowshoe hare. Journal of Parasitology 26: 334.

- 1944. Parasites of beavers, with a note on Paramphistomum castori Kofoid and Park, 1937 a synonym of Stichorchis subtriquetrus. American Midland Naturalist 31: 625-630. 1946. Incidence of worm parasites in Minnesota Mustelidae and host lists and keys to North American species. American Midland Naturalist 36: 494-509.

1947. Helminth parasites of rabbits of the genus Sylvilagus. Journal of Wildlife Management 11: 255-263.

1950. Helminths of Minnesota Canidae in relation to food habits, and a host list and key to the species reported from North America. American Midland Naturalist 32: 358-372.

ERICKSON, A. B., AND P. R. HIGHBY. 1942. Parasites of the woodland caribou. Journal of Parasitology 28: 423.

FARLEY, J. 1962. Schistosomatium douthitti (Cort, 1914) Prince, 1931 in Manitoba. Canadian Journal of Zoology 40: 131-133.

FAUST, E. C. 1919. A new trematode, Acanthatrium nycteridis, nov. gen., nov. spec., from the little brown bat. Transactions of the American Microscopical Society 38: 209-215. 
FEDYNICH, A. M., D. B. PENCE, AND R. L. URUBEK. 1986. Helminth fauna of beaver from central Texas. Journal of Wildlife Diseases 22: 579-582.

FELDMAN, D. B., J. A. MOORE, M. W. HARRIS, AND J. L. SELF. 1972. Characteristics of common helminths of the Virginia opossum (Didelphis virginiana) from North Carolina. Laboratory Animal Science 22: 183-189.

FENSTERMACHER, R. 1934. Further studies of diseases affecting moose. Bulletin 308, University of Minnesota Agricultural Experimental Station: $26 \mathrm{pp}$. 1937. Further studies of diseases affecting moose. II. Cornell Veterinarian 27: 25-37.

FENSTERMACHER, R., AND W. L. JELLISON. 1933. Diseases affecting moose. Bulletin 294, University of Minnesota Agricultural Experimental Station: 20 pp.

FENSTERMACHER, R., O. W. OLSEN, AND B. S. POMEROY. 1943. Some diseases of white-tailed deer in Minnesota. Cornell Veterinarian 33: 323-332.

FLEISCHMAN, R. W., AND R. A. SQUIRE. 1970. Verminous pneumonia in the California sea lion (Zalophus californianus). Pathologia Veterinaria 7: 89-101.

FLEMING, W. J., C. F. DIXON, AND J. W. LOVETT. 1977. Helminth parasites of river otters ( Lutra canadensis) from southeastern Alabama. Proceedings of the Helminthological Society of Washington 44: 131-135.

FLOOK, D. R., AND J. E. STENTON. 1969. Incidence and abundance of certain parasites in wapiti in the national parks of the Canadian Rockies. Canadian Journal of Zoology 47: 795-803.

FOLEY, G. L., W. I. ANDERSON, AND M. E. GEORGI. 1987. Eurytrema procyonis in a New York fox. Cornell Veterinarian 77: 168-171.

FONT, W. F. 1978. Ototrema schildti gen. et sp. n. (Trematoda: Lecithodendriidae) from the little brown bat, Myotis lucifugus in Wisconsin. Journal of Parasitology 64: 391392.

FOREYT, W. J., AND D. L. DRAWE. 1978. Anthelminthic activity of albendazole in white-tailed deer. American Journal of Veterinary Research 39: 1901-1903.

FOREYT, W. J., AND R. L. HUNTER. 1980. Clinical Fascioloides magna infection in sheep in Oregon on pasture shared by Columbian white- tailed deer. American
Journal of Veterinary Research 41: 15311532.

FOREYT, W. J., W. M. SAMUEL, AND A. C. TODD. 1977. Fascioloides magna in whitetailed deer (Odocoileus virginianus): observations on the pairing tendency. Journal of Parasitology 63: 1050-1052.

FOREYT, W. J., AND A. C. TODD. 1972. The occurrence of Fascioloides magna and Fasciola hepatica together in the livers of naturally infected cattle in south Texas, and the incidence of the flukes in cattle, whitetailed deer, and feral hogs. Journal of Parasitology 58: 1010-1011. 1973. Action of Oxyclozanide against adult $F$ ascioloides magna (Bassi, 1875) infections in white-tailed deer. Journal of Parasitology 59: 208-209.

1976. Effects of six fasciolicides against Fascioloides magna in white-tailed deer. Journal of Wildlife Diseases 12: 361-366.

FOREYT, W. J., A. C. TODD, AND K. FOREYT. 1975. Fascioloides magna (Bassi, 1875) in feral swine from southern Texas. Journal of Wildlife Diseases 11: 554-559.

FORRESTER, D. J., AND W. D. ROBERTSON. 1975. Helminths of rough- toothed dolphins, Steno bredanensis Lesson 1828, from Florida waters. Journal of Parasitology 61: 922.

FOSTER, A. O. 1939. Some helminths of the woolly opossum in Panama. Transactions of the American Microscopical Society 58: 185198.

FRANDSEN, J. C., AND A. W. GRUNDMANN. 1960. Brachylaime microti from the deer mouse in Utah. Journal of Parasitology 46: 314.

1961. Endoparasitism in isolated populations of rodents of the Lake Bonneville Basin, Utah. Journal of Parasitology 47: 391-396.

FRANKLIN, J., M. L. SIMMONS, AND G. E. COSGROVE. 1966. A pathogen survey in the Kansas cottontail. Bulletin of the Wildlife Disease Association 2: 52-53.

FYVIE, A. 1966. A review and current survey of the common diseases and parasites of wildlife in Ontario. Ontario Department of Lands and Forests Section Report (Wildlife) 63: 1-21.

GASH, S. L., AND W. L. HANNA. 1972. Occurrence of some helminth parasites in the muskrat, Ondatra zibethicus, from Crawford County, Kansas. Transactions of the Kansas Academy of Science 75: 251-254. 
GESINSKI, R. M., R. E. THOMAS, AND V. GALLICCHIO. 1964. Survey of Paragonimus in Ohio mink. Journal of Parasitology 50: 151.

GIBSON, D. J., AND E. A. HARRIS. 1979. The helminth parasites of cetaceans in the collection of the British Museum (Natural History), p. 309-324. In: G. Pilleri (ed) Investigations on Cetacea, No. 10.

GILBERTSON, D. E. 1977. Alaria arisaemoides (Trematoda): Occurrence in foxes from Minnesota. Journal of Parasitology 63: 162163.

GILFORD, J. H. 1954. A survey of muskrat helminths in Illinois. Journal of Parasitology 40: 702-703.

GLAZENER, W. C., AND F. F. KNOWLTON. 1967. Some endoparasites found in Welder Refuge deer. Journal of Wildlife Management 31: 595-597.

GOLDBERG, A. 1954. Parasites of skunks in the Beltsville, Maryland, area. Proceedings of the Helminthological Society of Washington 21: 29-34.

GRIFFITHS, H. J. 1962. Fascioloidiasis of cattle, sheep, and deer in northern Minnesota. Journal of the American Veterinary Medical Association 140: 342-347.

GRUNDMANN, A. W., AND J. C. FRANDSEN. 1960. Definitive host relation- ships of the helminth parasites of the deer mouse, Peromyscus maniculatus, in the Bonneville basin of Utah. Journal of Parasitology 46: 673-677.

GRUNDMANN, A. W., AND Y-H TSAI. 1967. Some parasites of the muskrat, Ondatra zibethicus osoyoosensis (Lord, 1863) Miller, 1912 from the Salt Lake Valley, Utah. Transactions of the American Microscopical Society 86: 139-144.

GUPTA, S. P. 1962a. On a new trematode, Quinqueserialis zibethicai sp. nov., of the sub-family Notocotylinae from the duodenum of muskrat ( Ondatra zibethica) from Canada. Indian Journal of Helminthology 14: 66-70. 1962b. A rediscription of Euparyphium melis (Shrank, 1788) Dietz, 1909 and Echinostoma revolutum (Froelich, 1802) Looss, 1899 parasitic in the intestine of mink (Mustela vison) and muskrat (Ondatra zibethica) from Canada. Indian Journal of Helminthology 14: 77-85.

HADWEN, S. 1916. A new host for Fasciola magna, Bassi, together with observations on the distribution of Fasciola hepatica, L., in Canada. Journal of the American

Veterinary Medical Association 2: 511-515.

- 1932. Helminth parasites of deer.

Journal of Parasitology 19: 83.

HALL, J. E., AND B. SONNENBERG. 1955.

Some helminth parasites of rodents from localities in Maryland and Kentucky. Journal of Parasitology 41: 640-641.

HALL, M. C. 1916. Hasstilesia tricolor (Stiles and Hassall, 1894), a common parasite of rabbits in the United States. Journal of the American Veterinary Medical Association 48: 453-456.

HAND, C., AND M. VOGE. 1952. Ariolimax columbianus, an intermediate host for Brachylaemus virginiana (Dickerson) (Trematoda: Digenea) in California. Journal of Parasitology 38: 57-58.

HARKEMA, R. 1936. The parasites of some North Carolina rodents. Ecological Monographs 6: 151-232. 1942. Pharyngostomoides procyonis n. g., n. sp. (Strigeida) a trematode from the raccoon in North Carolina and Texas. Journal of Parasitology 28: 117-122.

HARKEMA, R., AND G. C. MILLER. 1959. Studies on the helminths of North Carolina vertebrates. I. Procyotrema marsupiformis n. g., n. sp. (Strigeida: Diplostomatidae) from the pancreatic duct of the raccoon. Journal of Parasitology 45: 151-153.

1961. Parallelorchis diglossus n. g., n. sp., a trematode (Strigeida: Diplostomidae) from the Florida raccoon. Journal of Parasitology 47: 611-613.

1962. Helminths of Procyon lotor solutus from Cape Island, South Carolina. Journal of Parasitology 48: 333-335.

1964. Helminth parasites of the raccoon, Procyon lotor in the southeastern United States. Journal of Parasitology 50: 60-66.

HARLEY, J. P. 1972. Paragonimus kellicotti in Kentucky. American Midland Naturalist 88: 474-475.

HARLOW, R. F., AND F. K. JONES, JR. 1965. The white-tailed deer in Florida. Technical Bulletin No. 9, Florida Game and Fresh Water Fish Commission.

HARRIS, A. H., R. HARKEMA, AND G. C. MILLER. 1967. Maternal transmission of Pharyngostomoides procyonis Harkema, 1942 (Trematoda: Diplostomatidae). Journal of Parasitology 53: 1114-1115.

HARWOOD, P. D. 1939. Notes on Tennessee helminths. IV. North American trematodes of the subfamily Notocotylinae. Journal of 
the Tennessee Academy of Science 14: 421437.

HENDRICKS, D. P. 1973. Neoglyphe soricis (Pojmanska) (Trematoda) from Montana shrews. Journal of Parasitology 59: 488.

HERBER, E. C. 1942. Life history studies on two trematodes of the subfamily Notocotylinae. Journal of Parasitology 28: 179-193.

1955. Life history studies on Notocotylus urbanensis (Trematoda: Notocotylinae). Proceedings of the Pennsylvania Academy of Science 29: 267-275.

HERMAN, C. M. 1945. Some worm parasites of deer in California. California Department of Fish and Game Game Bulletin 31: 201-208.

HERMAN, C. M., P. M. BAUMAN, AND R. T. HABERMANN. 1957. The prevalence of Eurytrema procyonis Denton (Trematoda: Dicrocoeliidae) in some mammals from Maryland. Journal of Parasitology 43: 113114.

HOBERG, E. P., AND S. G. McGEE. 1982. Helminth parasitism in racoons, Procyon lotor hirtus Nelson and Goldman, in Saskatchewan. Canadian Journal of Zoology 60: 53-57.

HOFFMAN, G. L. 1955. Notes on the life cycle of Fibricola cratera (Trematoda: Strigeida). Journal of Parasitology 41: 327.

HOLLAND, J. B., JR. 1959. Liver flukes in the southeastern white- tailed deer. Proceedings of the 12th Annual Conference of the Southeastern Association of Game and Fish Commissioners. October 19-22, 1958. pp 224-227.

HOLLOWAY, H. L. 1966. Helminths of rabbits and opossums at Mountain Lake, Virginia. Bulletin of the Wildlife Disease Association 2: 38.

HOLMES, J. C. 1963. Helminth parasites of pine marten, Martes americana, from the District of MacKenzie. Canadian Journal of Zoology 41: 333.

HOLMES, J. C., AND R. PODESTA. 1968. The helminths of wolves and coyotes from the forested regions of Alberta. Canadian Journal of Zoology 46: 1193-1204.

HUFFMAN, J. E., AND L. R. PENNER. 1981. Helminths from the short- tailed shrew, Blarina brevicauda, in Connecticut with reference to the histopathology of Capillaria. Proceedings of the Helminthological Society of Washington 48: 209-213.

JACOBSON, H. A., R. L. KIRKPATRICK, AND R. B. HOLLIMAN. 1974. Emaciation and enteritis of cottontail rabbits infected with
Hasstilesia tricolor and observations on a fluke to fluke attachment phenomenon. Journal of Wildlife Diseases 10: 111-114.

JACOBSON, H. A., R. L. KIRKPATRICK, AND B. S. McGINNES. 1978. Disease and physiologic characteristics of two cottontail populations in Virginia. Wildlife Monograph No. 60.

JAMESON, D. K. 1959. A survey of the parasites of five species of bats. Southwestern Naturalist 4: 61-65.

JENKINS, E. AND A. W. GRUNDMANN. 1973. The parasitology of the ground squirrels of western Utah. Proceedings of the Helminthological ociety of Washington 40: 76-86.

JENSEN, D. N. 1972. The life history of Scaphiostomum pancreaticum McIntosh, 1934 (Trematoda: Brachylaemidae). Canadian Journal of Zoology 50: 201-204.

JILEK, R. 1977. Trematode parasites of the muskrat, Ondatra zibethica zibethica, in southern Illinois. Transactions of the I11inois State Academy of Science 70: 105107.

JOHNSON, A. D. 1968. Life history of Alaria marcianae (La Rue, 1917) Walton, 1949 (Trematoda: Diplostomatidae). Journal of Parasitology 54: 324-332.

1979. Morphology and life history of Alaria mustelae Bosma 1931 (Trematoda: Diplostomatidae) from Minnesota mustelids. Journal of Parasitology 65: 154160.

JOHNSTON, D. G., AND S. H. RIDGWAY. 1969. Parasitism in some marine mammals. Journal of the American Veterinary Medical Association 155: 1064-1072.

JORDAN, H. E., AND E. E. BYRD. 1958. Paragonimus in wild and domesticated animals in Georgia. Journal of Parasitology 44: 470 .

JORDAN, H. E., AND F. A. HAYES. 1959. Gastrointestinal helminths of raccoons (Procyon lotor) from Ossabaw Island, Georgia. Journal of Parasitology 45: 249252.

KAPLAN, E. H. 1964. Heterobilharzia americana Price, 1929, in the opossum from Louisiana. Journal of Parasitology 50: 797.

KENNEDY, M. J., M. W. LANKESTER, AND J. B. SNIDER. 1985. Paramphistomum cervi and Paramphistomum liorchis (Digenea: Paramphistomatidae) in moose, Alces alces, from Ontario, Canada. Canadian Journal of Zoology 63: 1207-1210. 
KINGSCOTE, A. A. 1947. Liver fluke control in the Burwash area. Report of the Ontario Veterinary College (1946): 34-35. 1950. Liver rot (fascioloidiasis) in ruminants. Canadian Journal of Comparative Medicine 14: 203-208. 1957. Fish, wildlife, and zoo animals. Report of the Ontario Veterinary College for 1956: 26-27.

KINSELLA, J. M. 1971. Growth, development, and intraspecific variation of Quinqueserialis quinqueserialis (Trematoda: Notocotylidae) in rodent hosts. Journal of Parasitology 57: 62-70.

KISTNER, T. P., D. WYSE, AND J. A. SCHMITZ. 1979. Pathogenicity attributed to massive infection of Nanophyetus salmincola in a cougar. Journal of Wildlife Diseases 15: 419-420.

KNAPP, S. E., AND J. N. SHAW. 1963. Occurrence of Fascioloides magna (Bassi) in Oregon cattle and deer. Journal of Parasitology 49: 339.

KNIGHT, I. M. 1951. Diseases and parasites of the muskrat (Ondatra zibethica) in British Columbia. Canadian Journal of Zoology 29: 188-214.

KNIGHT, R. A., AND I. PRATT. 1955. The life history of Allassogonoporus vespertilionis Macy and Acanthatrium oregonense Macy (Trematoda: Lecithodendriidae). Journal of Parasitology 41: 248-255.

KOCAN, A. A., K. WALDRUP, AND G. KUBAT . 1982. Scanning electron microscope observations of adult Heterobilharzia americana. Proceedings of the Oklahoma Academy of Science 62: 63-64.

KRUIDENIER, F. J. AND V. GALLICCHIO. 1959. The orthography of the Brachylaimidae (Joyeux and Foley, 1930); Brachylaime microti sp. nov.; B. rauschi McIntosh, 1950; and an addendum to Dollfus' (1935) list of Brachylaime (Trematoda: Digenea). Transactions of the American Microscopical Society 78: 428441.

KRULL, W. H. 1935. A note on the life history of Echinostoma coalitum Barker and Beaver, 1915 (Trematoda: Echinostomatidae). Proceedings of the Helminthological Society of Washington 2: 76.

KUNS, M. L., AND R. RAUSCH. 1950. An ecological study of helminths of some Wyoming voles (Microtus spp.) with a description of a new species of Nematospiroides (Heligmosomidae: Nematoda). Zoologica 35: 181-188.
KUNZ, T. H. 1968. Helminths from the red bat, Lasiurus borealis, in Iowa. American Midland Naturalist 80: 542-543.

LAMONTHE-ARGUMEDO, R. 1978.

Trematodes de mamiferos I. Redescription de Rhopalias macracanthus Chandler, 1932 y algunas consideraciones sobre el genero. Anales del Instituto de Biologia Universidad Nacional Autonoma de Mexico 49, Series Zoologia I: 25-34.

1980. Trematodes de mamiferos. II. Una especie nueva delgenero Dictyonograptus Travassos, 1919 (Trematoda:

Dicrocoeliidae). Anales del Institute de Biologia. Universidad Nacional Autonoma, de Mexico 51: Ser. Zoologia 1: 61-68. 1981. Hospederos definitivos e intermediarios de Paragonimus mexicanus, Miyasaki e Ishii, 1968 en Mexico. Anales del Institute de Biologia. Universidad Nacional Autonoma de Mexico 52, Ser Zoologia 1: 39-44.

- 1984. Nuevos datos sobre la distribucion geografica de Paragonimus mexicanus, y tres nuevos hospederos intermediarios en Mexico. Anales del Institute de Biologia. Universidad Nacional Autonoma de Mexico 55, Ser Zoologia 1: 85-89.

LAMOTHE-ARGUMEDO, R., J. CABALLERODELOYA, R. PINEDA-LOPEZ, AND O. MEAVE-GALLEGOS. 1985. Hallazgo de Paragonimus mexicanus en nuevo hospedero y una localidad en Mexico. Universidad y Ciencia 2: 41-45.

LAMOTHE-ARGUMEDO, R., R. P. LOPEZ, AND O. M. GALLEGOS. 1981. Infeccion natural de Paragonimus mexicanus en Didelphis virginiana californica, en Colima, Mexico. Anales del Institute de Biologia.

Universidad Nacional Autonoma de Mexico 52, Ser Zoologia 1: 45-50.

LANG, B. Z. 1977. Snail and mammalian hosts for Fasciola hepatica in eastern Washington. Journal of Parasitology 63: 938-939.

LANG, B. Z., AND N. O. DRONEN. 1972.

Prosthodendrium naviculum Macy, 1936, and Cephalophallus obscurus Macy and Moore, 1954 (Trematoda:

Lecithodendriidae) from Mustela vison Schreber in Michigan. Journal of Parasitology 58: 384.

LANG, B. Z., L. N. GLEASON, AND B. R. FORD 1968. Morphological differences between population samples of Metagonimoides oregonensis Price, 1931 (Trematoda: Heterophyidae) from Oregon and North 
Carolina. Journal of the Elisha Mitchell

Scientific Society 84: 281-284.

LANG, B. Z., AND H. D. VAIL. 1970.

Metagonimoides oregonensis Price, 1931

(Trematoda: Heterophyidae) from Mustela vison Schreber in Michigan. Journal of

Parasitology 56: 1031-1032.

LANKESTER, M. W. 1972. Diseases of moose in southeastern Manitoba 8th North American Moose Conference Workshop, Thunder Bay, Ontario. Ontario Ministry of Natural

Resources, Toronto. p. 42-59.

-- 1974. Parelaphostrongylus tenuis

(Nematoda) and Fascioloides magna

(Trematoda) in moose of southeastern

Manitoba. Canadian Journal of Zoology 52: 235-239.

LANKESTER, M. W., J. B. SNIDER, AND

R.E.JERRARD. 1979. Annual maturation

of Paramphistomum cervi (Trematoda:

Paramphistomatidae) in moose, Alces alces

L. Canadian Journal of Zoology 57: 23552357.

LARSON, O. R., AND W. C. SCHARF. 1975. New helminth records from Minnesota mammals. Proceedings of the

Helminthological Society of Washington 42: 174-175.

LaRUE, G. R., AND D. J. AMEEL. 1937. The distribution of Paragonimus. Journal of Parasitology 23: 382-388.

LaRUE, G. R., AND G. H. BARONE. 1927.

Studies on the trematode family Strigeidae

(Holostomidae). Alaria oregonensis, n. sp.

Journal of Parasitology 14: 124.

-- 1932. Alaria oregonensis from the coyote

(Trematoda: Alariidae). Transactions of the

American Microscopical Society 51: 199-

208.

LaRUE, G. R., AND N. J. BOSMA. 1927. Studies on the trematode family Strigeidae

(Holostomidae). Neodiplostomum lucidum

n. sp. Journal of Parasitology 14: 124-125.

LaRUE, G. R., AND E. W. TOWNSEND. 1927.

Studies on the trematode family Strigeidae

(Holostomidae). Alaria nasuae sp. nov.

Journal of Parasitology 14: 124.

- 1932. A morphological study of Alaria

nasuae LaRue and Townsend (Trematoda:

Alariidae). Transactions of the American

Microscopical Society 51: 252-263.

LAW, R. G., AND A. H. KENNEDY. 1932.

Parasites of fur-bearing animals. Bulletin of the Ontario Department for Game Fish 4: $1-30$.
LEE, HONG-FANG. 1962. Life history of

Heterobilharzia americana Price, 1929, a

schistosome of the raccoon and other mammals in southeastern United States. Journal of Parasitology 48: 728-739.

LEIBY, P. D. 1961. Intestinal helminths of some Colorado mammals. Journal of Parasitology 47: 311.

LEIBY, P. D., P. J. SITZMANN, AND D. C. KRITSKY. 1971. Studies on helminths of North Dakota. II. Parasites of the badger, Taxidea taxus (Schreber). Proceedings of the Helminthological Society of Washington 38: 225-228.

LEIDY, J. 1858. Contributions to helminthology. Proceedings of the Academy of Natural Science of Philadelphia 10 (sec, 2V2): 110112.

- 1888. Trematodes of the muskrat. Proceedings of the Academy of Natural Science of Philadelphia 40 ( $\sec 3 \mathrm{v} 18)$ : 126. 1890. Notices of Entozoa. Proceedings of the Academy of Natural Science of Philadelphia 42: 410-418.

LEIGH, W. H. 1940. Preliminary studies on parasites of upland game birds and furbearing mammals in Illinois. Illinois Natural History Survey Bulletin 21: 181194.

1958. Carneophallus turgidus sp. nov. (Trematoda: Microphallidae) from the raccoon, Procyon lotor, in south Florida. Journal of Parasitology 44: 100-102.

LEVIN, N. L. 1957. The occurrence of Panopistus pricei in Illinois. Journal of Parasitology 43: 379.

LEVIN, P. P. 1934. Pathological examination of game. Annual Report of the New York State Conservation Department 23: 285289.

LLEWELLYN, L. M., AND C. O. HANDLEY. 1945. The cottontail rabbits of Virginia. Journal of Mammalogy 26: 379-390.

LOCKE, L. N., AND E. E. BROWN. 1965. Occurrence of the fluke, Procyotrema marsupiformis Harkema and Miller, 1959, in a Maryland raccoon. Journal of Parasitology 51: 355.

LOCKER, B. 1953. Parasites of bison in northwestern U.S.A. Journal of Parasitology 39: 58-59.

LOFTIN, H. 1960. An annotated check-list of trematodes and cestodes and their vertebrate hosts from northwest Florida. Quarterly Journal of the Florida Academy of Science 23: 302-314. 
LOKEN, K. I., J. C. SCHLOTTHAUER, H. J. KURTZ, AND P. D. KARNS. 1965.

Pneumostrongylus tenuis in Minnesota moose (Alces alces). Bulletin of the Wildlife Disease Association 1: 7.

LONGHURST, W. M., AND J. R. DOUGLAS. 1953. Parasite interrelationships of domestic sheep and Columbian black-tailed deer. Proceedings of the Eighteenth North American Wildlife Conference 18: 168-188.

LOOSS, A. 1885 . Beitrage zur Kenntniss der Trematoden. Distomum palliatum, sp. n., und $D$. reticulatum, sp. n. Z. wiss. Zool. xli: 390-446.

LOTZ, J. M., AND W. F. FONT. 1983. Review of the Lecithodendriidae (Trematoda) from Eptesicus fuscus in Wisconsin and Minnesota. Proceedings of the Helminthological Society of Washington 50: 83-102.

LUMSDEN, R. D., AND C. A. WINKLER. 1962. The opposum, Didelphis virginiana (Kerr), a host for the cyathocotylid trematode Linstowiella szidati (Anderson, 1944) in Louisiana. Journal of Parasitology 48: 503.

LUMSDEN, R. D., AND J. A. ZISCHKE. 1961. Seven trematodes from small mammals in Louisiana. Tulane Studies in Zoology 9: 7785.

MacKINNON, B. M., AND M. D. B. BURT. 1978. Platyhelminth parasites of muskrats (Ondatra zibethica) in New Brunswick. Canadian Journal of Zoology 56: 350-354.

MACY, R. W. 1932. New bat trematodes of the genera Plagiorchis, Limatulum, and Dicrocoelium . Journal of Parasitology 18: 28-33.

1935a. Gyrabascus brevigastrus, new genus, new species, a bat trematode, with a note on Distomum mehelyi Modlinger. Journal of Parasitology 21: 413-415. -- 1935b. A new trematode, Limatulum gastroides (Lecithodendriidae), from the little brown bat, Myotis lucifugus .

Proceedings of the Helminthological Society of Washington 2: 74-75.

1936a. A new genus and species of trematode from the little brown bat and a key to the genera of Pleurogenetinae. Proceedings of the United States National Museum 83: 321-324.

- 1936b. Three new trematodes of Minnesota bats with a key to the genus Prosthodendrium. Transactions of the American Microscopical Society 55: 352 359.

1936c. A new bat trematode,
Lecithodendrium breckenridgei, with a key to the species of the genus. Zentralbl.

Bakteriol. Parasitenkd. 136: 236-237 -- 1938. A new species of trematode,

Prosthodendrium travassosi,

(Lecithodendriidae) from a Minnesota bat. In A. Neiva, ed. Livro Jubilar Professor Lauro Travassos. Instituto Oswaldo Cruz, Rio de Janeiro, Brazil. pp 291-292.

-- 1939. A new trematode Acanthatrium oregonense (Lecithodendriidae), from bats of the genus Myotis. American Midland Naturalist 22: 640-641.

1940a. A new trematode, Myotitrema asymmetrica, n. g., n. sp., (Lecithodendriidae) from the little brown bat. Journal of Parasitology 26: 83-84. 1940b. Description of three new trematodes with notes on other species of Acanthatrium (Lecithodendriidae), and a key to the genus. Journal of Parasitology 26: 279-283.

-1940c. A new species of trematode, Allassogonoporus vespertilionis (Lecithodendriidae), from an Oregon bat, Myotis californicus caurinus Miller. Transactions of the American Microscopical Society 59: 48-51. - 1947. Parasites found in certain Oregon bats with the description of a new cestode, Hymenolepis gertschi. The American Midland Naturalist 37: 375-378. 1960. The life cycle of Plagiorchis vespertilionis parorchis, $\mathrm{n}$. spp., (Trematoda: Plagiorchiidae), and observations on the effects of light on the emergence of the cercaria. Journal of Parasitology 46: 337 345 .

MACY, R. W., AND D. J. MOORE. 1954. On the life cycle and taxonomic relations of Cephalophallus obscurus n. g., n. sp., an intestinal trematode (Lecithodendriidae) of mink. Journal of Parasitology 40(3): 328335.

MALEK, E. A., L. R. ASH, H. F. LEE, AND M. D. LITTLE. 1961. Heterobilharzia infection in the dog and other mammals in Louisiana. Journal of Parasitology 47: 619-623.

MANTER, H. W., AND J. S. DEBUS. 1945. Two trematodes from a hibernating bat, Myotis californicus. Transactions of the American Microscopical Society 64: 297-299.

MAPES, C. R. 1950. The lancet fluke, a new parasite of the woodchuck. Cornell Veterinarian 40: 346-349. 
MAPES, C. R., AND D. W. BAKER. 1950. The white-tailed deer, a new host of Dicrocoelium dendriticum (Rudolphi, 1819) Looss, 1899 (Trematoda: Dicrocoeliidae). Cornell Veterinarian 40: 211-212.

MARGOLIS, L., AND G. C. PIKE. 1955. Some helminth parasites of Canadian Pacific whales. Journal of the Fisheries Research Board of Canada 12: 97-120

MARKER, G. E., AND T. C. CHENG. 1960. Intraspecific variations in Hasstilesia tricolor (Trematoda: Hasstilesiidae) and notes on the systematics of the family. Proceedings of the Pennsylvania Academy of Science 1934:191-198.

MARTIN, D. R. 1976. New host and distribution records of helminth parasites of the Mexican free-tail bat, Tadarida brasiliensis, from Texas and Louisiana. Proceedings of the Helminthological Society of Washington 43: 85-86.

MASON, J. JR. 1953. Brachylaima dolichodirus n. sp. from a shrew, Blarina brevidauda. Journal of the Tennessee Academy of Science 28(1): 38-42, 85.

MAZZOTTI, L., AND I. MIYAZAKI. 1965. The first record of adult lung flukes Paragonimus in Mexico (Trematoda: Troglotrematidae). Japanese Journal of Parasitology 14: 34-36.

McGEE, S. G. 1980. Helminth parasites of squirrels (Sciuridae in Saskatchewan. Canadian Journal of Zoology 58: 2040-2050.

McINTOSH, A. 1933. Dicrocoelium lasiuri n. sp.

(Trematoda, Digenea) from the liver of a Red Bat. Journal of Parasitology 20: 137138.

1934. Two new species of trematodes, Scaphiostomum pancreaticum n. sp. and Postharmostomum laruei n. sp., from the chipmunk. Proceedings of the Helminthological Society of Washington 1: $2-4$.

1935. New host records of parasites. Proceedings of the Helminthological Society of Washington 2: 80.

1937. A new trematode, Postharmostomum noveboracensis, n. sp. (Brachylaemidae), from a chipmunk. Proceedings of the Helminthological Society of Washington 4: 23-24.

1939. A new dicrocoeliid trematode, Eurytrema komareki, n. sp., from a white footed mouse. Proceedings of the Helminthological Society of Washington 6: 18-19.

1950. Brachylaima rauschi $\mathrm{n}$. sp. from an
Arctic lemming, Dicrostonyx groenlandicus rubricatus (Richardson, 1839). Journal of Parasitology 36 (Supplement 6): 20.

McINTOSH, A., AND G. E. McINTOSH. 1934. A new trematode, Notocotylus hassalli, n. sp. (Notocotylidae), from a meadow mouse. Proceedings of the Helminthological Society of Washington 1: 36-37.

1935. Additional notes on two bat parasites, Dicrocoelium lasiuri McIntosh, 1933 (Trematoda: Dicrocoeliidae) and Litomosa americana McIntosh, 1932 (Nematoda: Filariidae). Proceedings of the Helminthological Society of Washington 2: 60-62.

McKEEVER, S. 1958. Observations on Paragonimus kellicotti Ward from Georgia. Journal of Parasitology 44: 324-327. 1971. Zonorchis komareki (McIntosh, 1939) (Trematoda: Dicrocoeliidae) from Reithrodontomys humulis (Audubon and Bachman). Journal of Parasitology 57: 865.

McKENZIE, C. E., AND H. E. WELCH. 1979. Parasite fauna of the muskrat, Ondatra zibethica (Linnaeus, 1766), in Manitoba, Canada. Canadian Journal of Zoology 57 : 640-646.

McNEIL, M. A., M. E. RAU, AND F. MESSIER. 1984. Helminths of wolves (Canis lupus L.) from southwestern Quebec. Canadian Journal of Zoology 62: 1659-1660.

MEYER, M. C., AND J. R. REILLY. 1950. Parasites of muskrats in Maine. American Midland Naturalist 44: 467-477.

MIGAKI, G., D. VAN DYKE, AND R. C. HUBBARD. 1971. Some histopathological lesions caused by helminths in marine mammals. Journal of Wildlife Diseases 7: 281-289.

MILLER, G. C. 1984. Longevity of Pharyngostomoides procyonis (Strigeoidea: Diplostomatidae) in the raccoon, Procyon lotor. Journal of Parasitology 70: 284-285.

MILLER, G. C., AND R. HARKEMA. 1960. A new locality record for Heterobilharzia americana Price, 1929. Journal of Parasitology 46: 206.

1962. Studies on helminths of North Carolina vertebrates. II. Ascocotyle ampullacea sp. n. (Trematoda:

Heterophyidae) from raccoon. Journal of Parasitology 48: 427-428.

-- 1963. Helminths of North Carolina vertebrates III. The synonomy of Phagicola diminuta (Stunkard and Haviland, 1924) and Phagicola lageniformis Chandler, 1941 (Trematoda: Heterophyidae). Proceedings of 
the Helminthological Society of Washington 30: 107-110.

1964. Studies on helminths of North

Carolina vertebrates. V. Parasites of the mink, Mustela vison Schreber. Journal of Parasitology 50: 717-720.

1968. Helminths of some wild mammals in the southeastern United States.

Proceedings of the Helminthological Society of Washington 35: 118-125.

1970. Helminths of the opossum (Didelphis virginiana) in North Carolina.

Proceedings of the Helminthological Society of Washington 37: 36-39.

MILLER, G. C., R. L. PRICE, AND D. A. WILSON. 1974. Helminths of the shorttailed shrew, Blarina brevicauda, in North Carolina. Journal of Parasitology 60: 523524.

MILLER, M. J. 1940. A new trematode, Fibricola laruei, from the raccoon in Canada.

Canadian Journal of Research, Section D, Zoological Sciences 18: 333-335.

MIYAZAKI, I., AND Y. ISHII. 1968. Studies on the Mexican lung flukes, with special reference to a description of Paragonimus mexicanus sp. nov. (Trematoda:

Troglotrematidae). Japanese Journal of Parasitology 17: 445-453.

MIYAZAKI, I., T. KIFUNE, AND R. LAMOTHEARGUMEDO. 1980. Taxonomic and biological studies on the lung flukes of Central America. Department of Parasitology, School of Medicine, Fukuoka University. Occasional Publication No. 2.

MOORE, E. R., AND G. C. MOORE. 1947. The helminth parasites of cottontail rabbits in Alabama, with notes on the arthropod Linguatula serrata. Journal of Mammalogy 28: 279-284.

MORGAN, B. B., AND E. F. WALLER. 1940. A survey of the parasites of the Iowa cottontail (Sylvilagus floridanus mearnsi). Journal of Wildlife Management 4: 21-26.

MURRELL, K. D. 1965. Stages in the life cycle of Wardius zibethicus Barker, 1915. Journal of Parasitology 51: 600-604.

NEILAND, K. A. 1961. Suspected role of parasites in non-rookery mortality of fur seals ( Callorhinus ursinus). Journal of Parasitology 47: 732.

-- 1962. Two new species of

Prosthodendrium Dollfus, 1931 (Trematoda: Lecithodendriidae) from the little brown bat in southeastern Alaska. Journal of Parasitology 48: 400-401.
NETTLES, V. F., A. K. PRESTWOOD, AND W. R. DAVIDSON. 1975. Severe parasitism in an opossum. Journal of Wildlife Diseases 11 : 419-420.

NICKEL, P. A., AND M. F. HANSEN. 1967. Helminths of bats collected in Kansas, Nebraska and Oklahoma. American Midland Naturalist 78: 481-486.

NORRIS, K. S. 1966. Whales, dolphins and porpoises. University of California Press, Berkeley and Los Angeles.

OLEXIK, W. A., A. E. PERRY, AND W. E. WILHELM. 1969. Ectoparasites and helminth endoparasites of the tree squirrels of southwest Tennessee. Journal of the Tennessee Academy of Science 44: 4-6.

OLIVIER, L. 1938. A new trematode, Allassogonoporus marginalis, from the muskrat. Journal of Parasitology 24: 155160.

OLSEN, O. W. 1948. Wild rabbits as reservoir hosts of the common liver fluke, Fasciola hepatica, in southern Texas. Journal of Parasitology 34: 119-123.

-- 1949a. Incidence of helminth parasites in beavers from the Platte River in Weld County, Colorado. Journal of the ColoradoWyoming Academy of Science 4: 64. - 1949b. White-tailed deer as a reservoir host of the large American liver fluke. Veterinary Medicine 44: 26-30.

OLSEN, O. W., AND R. FENSTERMACHER. 1943. The helminths of North American deer with special reference to those of the white-tailed deer (Odocoileus virginianus borealis) in Minnesota. University of Minnesota Agriculture Experimental Station Technical Bulletin 159.

PARKER, M. V. 1950. Euryhelmis squamala (Rudolphi), 1819 reported from a racoon Journal of Parasitology 36: 89.

PATTON, S., A. RABINOWITZ, S. RANDOLPH, AND S. S. JOHNSON. 1986. A coprological survey of parasites of wild neotropical Felidae. The Journal of Parasitology 72: 517-520.

PEARSON, J. C. 1954. The life cycles of Alaria arisaemoides Augustine and Uribe, 1927, and Alaria canis LaRue and Fallis, 1936 (Trematoda: Diplostomidae), parasites of the red fox, Vulpes fulva (Desmarest). Journal of Parasitology 40: 37-38.

PENCE, D. B., AND W. P. MEINZER. 1979. Helminth parasitism in the coyote, Canis latrans, from the Rolling Plains of Texas. International Journal of Parasitology 9: 339-344. 
PENN, G. H. 1942. Parasitological survey of Louisiana muskrats. Journal of Parasitology 28: 348-349.

PENNER, L. R. 1938. Schistosomatium from the muskrat, Ondatra zibethica, in Minnesota and Michigan. Journal of Parasitology 24: 26.

1941. The status of Urotrema shillingeri Price, 1931 (Trematoda: Urotrematidae). Transactions of the American Microscopical Society 60: 359-364.

PENNER, L. R., C. F. HELMBOLDT, AND A. L. GRISWOLD. 1954. Eurytrema procyonis in a raccoon from Connecticut. Proceedings of the Helminthological Society of Washington 21: 34-35.

PETERSON, R. L. 1955. North American moose. University of Toronto Press, Toronto (see pp 181-192).

POOLE, B. C., K. CHADEE, AND T. A. DICK. 1983. Helminth parasites of pine marten, Martes americana (Turton), from Manitoba, Canada. Journal of Wildlife Diseases 19: 1013.

PREMVATI, G., AND T. D. BAIR. 1979.

Trematode parasites of the opossum, Didelphis virginiana, from Florida.

Proceedings of the Helminthological Society of Washington 46: 207-212.

PRESIDENTE, P. J. A., AND R. O. RAMSDEN. 1975. Paragonimus kellicotti infection in wild carnivores in southwestern Ontario: II. Histopathologic Features. Journal of Wildlife Diseases II: 364-375.

PRESTWOOD, A. K., F. E. KELLOGG, S. R. PURSGLOVE, AND F. A. HAYES. 1975. Helminth parasitisms among intermingling insular populations of white-tailed deer, feral cattle, and feral swine. Journal of the American Veterinary Medical Association 166: 787-789.

PRESTWOOD, A. K., J. F. SMITH, AND W. E. MAHAN. 1970. Geographic distribution of Gongylonema pulchrum, Gongylonema verrucosum, and Paramphistomum liorchis in white-tailed deer of the south-eastern United States. Journal of Parasitology 56: 123-127.

PRICE, E. W. 1929. A synopsis of the trematode family Schistosomidae with descriptions of new genera and species. Proceedings of the U. S. National Museum 75: 1-39. -- 1931a. Metagonimoides oregonensis , a new trematode from a raccoon. Journal of the Washington Academy of Science 21: 405-407.

$$
\text { 1931b. Four new species of trematode }
$$

worms from the muskrat, Ondatra zibethica, with a key to the trematode parasites of the muskrat. Proceedings of the United States National Museum 79: 1-13.

1932. The trematode parasites of marine mammals. Proceedings of the United States National Museums 81: L68.

1934. A new trematode from a beaver.

Proceedings of the Helminthological Society of Washington 1: 1-2.

1943. A redescription of Heterobilharzia americana Price (Trematoda:

Schistosomatidae). Proceedings of the Helminthological Society of Washington 10: 85-86.

PRICE, H. F. 1939. Life history of Schistosomatium douthitti (Cort). American Journal of Hygiene 13: 685-727.

PRICE, R. L., AND D. M. HARMAN. 1983. Helminths from the raccoon, Procyon lotor litoreus Nelson and Goldman 1930, on St. Catherines Island, Georgia. Proceedings of the Helminthological Society of Washington 50: 343-344.

PURSGLOVE, S. R., A. K. PRESTWOOD, T. R. RIDGEWAY, AND F. A. HAYES. 1977. Fascioloides magna infection in whitetailed deer of southeastern United States. Journal of the American Veterinary Medical Association 171: 936-938.

RAMSDEN, R. O., AND P. J. A. PRESIDENTE. 1975. Paragonimus kellicotti infection in wild carnivores in southwestern Ontario: I. Prevalence and gross pathological features. Journal of Wildlife Diseases 11: 136-141.

RANKIN, J. S. 1946. Helminth parasites of birds and mammals in western Massachusetts. The American Midland Naturalist 35: 756768.

RANSOM, B. H. 1920. Synopsis of the trematode family Heterophyidae with descriptions of new genus and five new species. Proceedings of the United States National Museum 57: 527-573.

RAUSCH, R. 1946a. The raccoon, a new host for Microphallus sp., with additional notes on $M$. ovatus from turtles. Journal of Parasitology 32: 208-209. 1946b. Parasites of Ohio muskrats. Journal of Wildlife Management 10: 70. -- 1952a. Helminths from the round-tailed muskrat, Neofiber alleni nigrescens Howell, with descriptions of two new species. Journal of Parasitology 38: 151-156. -1952b. Studies on the helminth fauna of Alaska. XI. Helminth parasites of microtine rodents-taxonomic 
considerations. Journal of Parasitology 38 : 415-444.

- 1953. Studies on the helminth fauna of Alaska. XIII. Disease in the sea otter, with special reference to helminth parasites.

Ecology 34: 584-604.

1959. Studies on the helminth fauna of

Alaska. XXXVI. Parasites of the wolverine, Gulo gulo L., with observations on the biology of Taenia twitchelli Schwartz, 1924. Journal of Parasitology 45: 465-484. -- 1962. Helminths of the shrew-mole Neurotrichus gibbsii (Baird) in Oregon. Journal of Parasitology 48: 813-817.

RAUSCH, R. L., AND F. H. FAY. 1966. Studies on the helminth fauna of Alaska. XLIV. Revision of Ogmogaster Jagerskiold, 1891, with a description of $\mathrm{O}$. pentalineatus $\mathrm{sp}$. $\mathrm{n}$. (Trematoda: Notocotylidae). Journal of Parasitology 52: 26-38.

RAUSCH, R. L., F. H. FAY, AND F. S. L. WILLIAMSON. 1983. Helminths of the arctic fox, Alopex lagopus (L.), in Greenland. Canadian Journal of Zoology 61: 1847-1851.

RAUSCH, R., AND B. LOCKER. 1951. Studies on the helminth fauna of Alaska II. On some helminths parasitic in the sea otter, Enhydra lutris (L.). Proceedings of the Helminthological Society of Washington 18: 77-81.

RAUSCH, R. L., AND D. W. RICE. 1970.

Ogmogaster trilineatus sp. n. (Trematoda:

Notocotylidae) from the fin whale, Balaenoptera physalus L. Proceedings of the Helminthological Society of Washington 37: 196-200.

RAUSCH, R., AND J. D. TINER. 1948. Studies on the parasitic helminths of the north central States. 1. Helminths of Sciuridae. The American Midland Naturalist 39: 728747.

1949. Studies on the parasitic helminths of the north central States. II. Helminths of voles (Microtus spp.) preliminary report. The American Midland Naturalist 41: 665694.

RAUSCH, R., AND F. S. L. WILLIAMSON. 1959. Studies on the helminth fauna of Alaska. XXXIV. The parasites of wolves, Canis lupus L.. Journal of Parasitology 45: 395403.

READ, C. P. 1948. Strigeids from Texas mink with notes on the genus Fibricola Dubois. Transactions of the American Microscopical Society 67: 165-168.
REYNOLDS, B. D. 1938. Brachylaemus peromysci $\mathrm{n}$. sp. (Trematoda) from the deer mouse. Journal of Parasitology 24: 245-248.

RICE, D. W., AND A. A. WOLMAN. 1971. The life history and ecology of the gray whale (Eschrichtius robustus). American Society of Mammalogists Special Publication No. 3: $140 \mathrm{pp}$.

RICE, E. W., AND O. B. HECK. 1975. A survey of the gastrointestinal helminths of the muskrat, Ondatra zibethicus, collected from two localities in Ohio. Ohio Journal of Science 75: 263-264.

RIDER, C. L., AND R. W. MACY. 1947. Preliminary survey of the helminth Hymenolepis ondatrae n. sp. Transactions of the American Microscopical Society 66: 176-181.

RIDGWAY, S. H., AND M. D. DAILEY. 1972. Cerebral and cerebellar involvement of trematode parasites in dolphins and their possible role in stranding. Journal of Wildlife Diseases 8: 33-43.

RIDGWAY, S. H., AND D. G. JOHNSTON. 1965. Two interesting disease cases in wild cetaceans. American Journal of Veterinary Research 26: 771-775.

RIGBY, M. D., AND W. THRELFALL. 1981. Helminth parasites of the muskrat (Ondatra zibethicus (L)) in Newfoundland. Canadian Journal of Zoology 59: 2172-2176.

ROBINSON, E. J., JR. 1959. New hosts for Hasstilesia tricolor and $H$. texensis (Trematoda: Brachylaimidae), and an emended diagnosis for the latter.

Transactions of the American Microscopical Society 78: 317-322.

RODENBURG, G. W. AND D. B. PENCE. 1978. Circulation of helminth species in a rodent population from the high plains of Texas. Occasional Papers of the Texas Technical University Museum No. 56: 1-9.

ROWAN, W. B. 1955. The life cycle and epizootiology of the rabbit trematode, Hasstilesia tricolor (Stiles and Hassall, 1894) Hall, 1916 (Trematoda:

Brachylaemidae). Transactions of the American Microscopical Society 74: 1-21.

SAMUEL, W. M., M. W. BARRETT, AND G. M. LYNCH. 1976. Helminths in moose of Alberta. Canadian Journal of Zoology 54: 307-312.

SAMUEL, W. M., AND W. A. LOW. 1970. Parasites of the collared peccary from Texas. Journal of Wildlife Diseases 6: 1623. 
SAMUEL, W. M., S. RAMALINGAM, AND L. N. CARBYN. 1978. Helminths in coyotes (Canis latrans Say), wolves (Canis lupus L.), and red foxes (Vulpes vulpes L.) of southwestern Manitoba. Canadian Journal of Zoology 56: 2614-2617.

SAMUEL, W. M., AND D. O. TRAINER. 1969. A technique for survey of some helminth and protozoan infections of white-tailed deer. Journal of Wildlife Management 33: 888894.

SAOUD, M. F. A. 1964. On a new trematode, Tremajoannes buckleyi gen. et sp. nov. (Lecithodendriidae) from Central American bats with some notes on Phaneropsolus orbicularis (Diesing, 1850) Braun, 1901. Journal of Helminthology 38: 97-108.

SAWYER, T. K. 1958. Metagonimoides oregonensis Price, 1931 from a Georgia raccoon with a note on Sellacotyle mustelae Wallace, 1935. Journal of Parasitology 44: 63.

\section{The American otter, Lutra} canadensis vaga, as a host for two species of trematodes previously unreported from North America. Proceedings of the Helminthological Society of Washington 28: 175-176.

SCHAD, G. A. 1954. Helminth parasites of mice in northeastern Quebec and the coast of Laborador. Canadian Journal of Zoology 32: 215-224.

\section{Helminths recovered from deer} mice, Peromyscus maniculatus, trapped in the Morgan Arboretum, Macdonald College, province of Quebec. Canadian Journal of Zoology 34: 208.

SCHAFFER, G. D., W. R. DAVIDSON, V. F. NETTLES, AND E. A. ROLLOR, III. 1981. Helminth parasites of translocated raccoons (Procyon lotor) in the southeastern United States. Journal of Wildlife Diseases 17: 217-227.

SCHLEGEL, M. W., S. E. KNAPP, AND R. E. MILLEMANN. 1968. "Salmon poisoning" disease. V. Definitive hosts of the trematode vector, Nanophyetus salmincola . Journal of Parasitology 54: 770-774.

SCHROEDER, R. E., AND W. H. LEIGH. 1965. The life history of Ascocotyle pachycystis sp. n., a trematode (Digenea: Heterophyidae) from the raccoon in south Florida. Journal of Parasitology 51: 594-599.

SCHROEDER, R. J., C. A. DELLI QUADRI, R. W McINTYRE, AND W. A. WALKER. 1973.

Marine mammal disease surveillance program in Los Angeles county. Journal of the American Veterinary Medical Association 163: 580-581.

SEALANDER, J. A. 1943. Notes on some parasites of the mink in southern Michigan. Journal of Parasitology 29: 361-362.

SEAMSTER, A., AND A. L. STEVENS. 1948. A new species of Prosthodendrium (Trematoda: Lecithodendriidae) from the large brown bat. Journal of Parasitology 34: 108-110.

SEESEE, F. M., M. C. STERNER, AND D. E. WORLEY. 1983. Helminths of the coyote (Canis latrans Say) in Montana. Journal of Wildlife Diseases 19: 54-55.

SELF, J. T., AND T. J. McKNIGHT. 1950. Platyhelminths from fur bearers in the Wichita Mountains Wildlife Refuge, with especial reference to Oochoristica spp. American Midland Naturalist 43: 58-61.

SENGER, C. M. 1953. Xiphidiotrema lockerae, gen. et sp. nov. (Trematoda:

Troglotrematidae) from shrews in the northwestern United States. Journal of Parasitology 39: 341-343.

1963. Some parasites of Montana deer. Montana Wildlife Autumn: 5-13.

SENGER, C. M., AND J. W. BATES. 1957. The occurrence of Hymenolepis evaginata and $H$. ondatrae in Utah muskrats. Proceedings of the Helminthological Society of Washington 24: 141-142.

SENGER, C. M., AND R. W. MACY. 1952. Helminths of northwest mammals. Part III. The description of Euryhelmis pacificus $\mathrm{n}$. sp., and notes on its life cycle. Journal of Parasitology 38: 481-486.

SENGER, C. M., AND K. A. NEILAND. 1955. Helminth parasites of some fur-bearers of Oregon. Journal of Parasitology 41: 637638.

SHAW, J. N. 1947. Some parasites of Oregon wild life. Station Technical Bulletin 11, Oregon State College. Agricultural Experimental Station, Corvallis.

SHOOP, W. L., AND K. C. CORKUM. 1982. Progenesis reconsidered in Ribeiroia ondatrae Babero, 1972 nec Price, 1931, a synonym of Gyrosoma singulare (Trematoda: Psilostomidae). Journal of Parasitology 68: 424.

SHORT, R.B AND A. I. GROSSMAN. 1986. Chromosomes of Heterobilharzia americana (Digenea: Schistosomatidae) from Texas. Journal of Parasitology 72: 807-809.

SHULTS, L. M. 1978. Pricetrema phocae and Pricetrema eumetopii spp. n. (Trematoda: Heterophyidae) from pinnipeds in the North 
Pacific. Canadian Journal of Zoology 56 : 382-385.

1982. Helminths of the spotted seal, Phoca largha, from the Bering sea. Journal of Wildlife Diseases 18: 59-62.

SMITH, C. F. 1954. Studies on Quinqueserialis hassalli and taxonomic considerations of the species of Quinqueserialis (Trematoda:

Notocotylidae). Journal of Parasitology 40: 209-215.

SMITH, F. R. 1938. Muskrat investigations in Dorchester County, MD., 1930-34. U. S. Department of Agriculture Circular No.474: 1-24.

SMITH, F. R., AND W. THRELFALL. 1973.

Helminths of some mammals from

Newfoundland. The American Midland Naturalist 90: 215-218.

SMITH, H. J. 1978. Parasites of red foxes in New Brunswick and Nova Scotia. Journal of Wildlife Diseases 14: 366-370.

SMITH, H. J., AND R. McG. ARCHIBALD. 1967. On the incidence of gastrointestinal parasites in Nova Scotia beaver. Canadian Journal of Zoology 45: 659-661.

SMITH, J. D., E. M. ADDISON; D. G. JOACHIM; AND L. M. SMITH. 1986. Helminth parasites of Canada lynx (Felis canadensis) from Northern Ontario. Canadian Journal of Zoology 64: 358-364.

SMITH, R. J. 1958. The miracidium of Wardius zibethicus (Trematoda:

Paramphistomatidae). Journal of Parasitology 44: 195.

SOGANDARES-BERNAL, F. 1961. Sellacotyle vitellosa, a new troglotrematid trematode from the mink in Louisiana. Journal of Parasitology 47: 911-912.

STAFFORD, J. 1905. Trematodes from Canadian vertebrates. Zoologischer Anzeiger 28: 681694.

STELFOX, J. G. 1962. Liver, lungs and larvae. Parasites and diseases in moose, deer and elk in Alberta. Alberta Lands, Forests, and Wildlife Annual Report 5: 5-12.

STILES, C. W., AND A. HASSALL. 1894a. A preliminary catalogue of the parasites contained in the collections of the United States Bureau of Animal Industry, Lnited States Army Medical Museum, Biological Department of the University of Pennsylvania (coll. Leidy) and in coll. Stiles and coll. Hassall. Veterinary Magazine 1: 245-253.

1894b. A new species of intestinal fluke (Distoma tricolor) in the cotton-tail rabbit (Lepus sylvaticus Bachman) and in the
Northern Hare (L. americanus Erxleben).

Veterinary Magazine 1: 729-737.

STOCK, T. M., AND M. W. BARRETT. 1983. Helminth parasites of the gastrointestinal tracts and lungs of moose (Alces alces) from Cypress Hills, Alberta, Canada.

Proceedings of the Helminthological Society of Washington 50: 246-251.

STONE, J. E., AND D. B. PENCE. 1978. Ecology of helminth parasitism in the bobcat from west Texas. Journal of Parasitology 64 : 295-302.

STRINGER, R. P., R. HARKEMA, AND G. C. MILLER. 1969. Parasites of rabbits in North Carolina. Journal of Parasitology 55: 328.

STROHLEIN, D. A., AND B. M. CHRISTENSEN. 1983. Metazoan parasites of the eastern cottontail rabbit in western Kentucky. Journal of Wildlife Diseases 19: 20-23.

STROUD, R. K., AND M. D. DAILEY. 1978. Parasites and associated pathology observed in pinnipeds stranded along the Oregon coast. Journal of Wildlife Diseases 14: 292-298.

STUNKARD, H. W. 1947. A dicrocoeliid trematode, Eurytrema vulpis n. sp. provis., from the pancreatic ducts of the red fox. Journal of Parasitology 33: 459-466.

STUNKARD, H. W., AND C. H. ALVEY. 1929. A new liver fluke, Zalophotrema hepaticum, from the California sea-lion, Zalophus californianus. Journal of Parasitology (abstract) 16: 106.

1930. The morphology of Zalophotrema hepaticum, with a review of the trematode family Fasciolidae. Parasitology 22: 326333.

STUNKARD, H. W., AND C. B. HAVILAND. 1924. Trematodes from the rat. American Museum of Novitities 126: 1-10.

STUNKARD, H. W., J. R. UZMANN. 1955. The kiliifish, Fundulus heteroclitus, second intermediate host of the trematode, Ascocotyle ( Phagicola) diminuta. Biological Bulletin 109: 475-483.

SWALES, W. E. 1933. A review of Canadian helminthology: II. Additions to Part I, as determined from a study of parasitic helminths collected in Canada. Canadian Journal of Research, Section D, Zoological Sciences 8: 478-482.

1935. The life cycle of Fascioloides magna (Bassi, 1875), the large liver fluke of ruminants, in Canada with observations on the bionomics of the larval stages and the intermediate hosts, pathology of 
Fasciloidiasis magna, and control measures. Canadian Journal of Research, Section D, Zoological Sciences 12: 177-215. -- 1936. Further studies on Fascioloides magna (Bassi, 1975) Ward, 1917, as a parasite of ruminants. Canadian Journal of Research, Section D, Zoological Sciences 14: 83-95.

SWANSON, D. O. 1960. A study of the helminth parasites of the George Reserve deer herd. Wildlife Review 98: 74 (abstract).

SWANSON, G., AND A. B. ERICKSON. 1946. Alaria taxideae n. sp., from the badger and other mustelids. Journal of Parasitology 32: 17-19.

SWEATMAN, G. K. 1952. Endoparasites of muskrats in the vicinity of Hamilton, Ontario. Journal of Mammalogy 33: 248250

SWEENEY, J. C. 1974. Common diseases of pinnipeds. Journal of the American Veterinary Medical Association 165: 805810.

SWEENEY, J. C., AND W. G. GILMARTIN. 1974. Survey of diseases in free-living California sea lions. Journal of Wildlife Diseases 10: 370-376.

THATCHER, V. E. 1967. Paragonimus in some wild and domestic animals of Panama. Transactions of the American Microscopical Society 86: 335-336.

THORNTON, J. E., R. R. BELL, AND M. J. REARDON. 1974. Internal parasites of coyotes in southern Texas. Journal of Wildlife Diseases 10: 232-236.

THREFALL, W. 1967. Parasites of moose (Alces alces) in Newfoundland. Journal of Mammalogy 48: 668-669.

TINER, J. D., AND T. H. CHIN. 1948. The occurrence of Ascaris lumbricoides L. 1758 in the muskrat, Ondatra zibethica L. Journal of Parasitology 34: 253

TURNER, H. M., AND S. McKEEVER. 1980. Ityogonimus scalopi sp. n. (Trematoda: Brachylaemidae) from the eastern mole, Scalopus aquaticus (Linnaeus 1758). Journal of Parasitology 66: 823-824.

UBELAKER, J. E. 1966. Parasites of the gray bat, Myotis grisescens, in Kansas. American Midland Naturalist 75: 199-204. 1971. Parasites of the evening bat, Nycteceius humeralis, in Iowa. Texas Journal of Science 22: 425-427.

UBELAKER, J. E., AND M. D. DAILEY. 1966. Taxonomy of the genus Brachylaima Dujardin (Trematoda: Digenea) with description of $B$. chiapensis sp. n. from
Peromyscus guatemalensis in Mexico. Journal of Parasitology 52: 1062-1065.

UBELAKER, J. E. AND T. H. KUNZ. 1971. Parasites of the evening bat, Nycticeius humeralis, in Iowa. Texas Journal of Science 22: 425-427.

VILLELLA, J. B. 1953. Retinella identata (Say), a first intermediate host of Entosiphonus thompsoni Sinitsin (1931) (Trematoda: Brachylaemidae). Journal of Parasitology 39: 667.

- 1954. Ventridens ligera, a new first intermediate host of Panopistus pricei Sinitsin, 1931 (Trematoda:

Brachylaimatidae). Journal of Parasitology 40: 470-472.

WALKER, M. L., AND W. W. BECKLUND. 1970. Checklist of the internal and external parasites of deer, Odocoileus hemionus and $O$. virginianus, in the United States and Canada. Index Catalogue for Medical Veterinary Zoology Specimens, Publication No. 1. United States Department of Agriculture.

WALLACE, F. G. 1931. Lung flukes of the genus Paragonimus in American mink. Journal of the Veterinary Medical Association 31: 229234. 1932. The life history of Troglotrema mustelae n. sp. (abstract). Journal of Parasitology 19: 164.

1934. Parasites collected from the moose, Alces americanus, in northern Minnesota. Journal of the American Veterinary Medical Association 37: 770-775. 1935. A morphological and biological study of the trematode, Sellacotyle mustelae n. g., n. sp.. Journal of Parasitology 21: 143166.

WALLACE, F. G., AND L. R. PENNER. 1939. A new liver fluke of the genus Opisthorchis. Journal of Parasitology 25: 437-440.

WALLACE, R. A., AND B. G. WILSON. 1965. Metagonimoides oregonensis Price, 1931 (Trematoda: Heterophyidae) from the raccoon in middle Tennessee. Journal of Parasitology 51: 391.

WARD, J. W. 1946. A preliminary study of the occurrence of internal parasites of animals in Mississippi. Proceedings of the Helminthological Society of Washington 13: 12-14.

WATSON, T. G. 1981. Growth of Metorchis conjunctus (Cobbold, 1860) Looss, 1899 (Trematoda: Opisthorchiidae) in the bile ducts of various definitive hosts. Canadian Journal of Zoology 59: 2014-2019. 
WATSON, T. G., V. F. NETTLES, AND W. R. DAVIDSON. 1981. Endoparasites and selected infectious agents in bobcats ( Felis rufus ) from West Virginia and Georgia. Journal of Wildlife Diseases 17: 547-554.

WEBSTER, G. A., AND R. W. WOLFGANG. 1956. Alaria canadensis sp. nov. and Euryhelmis pyriformis sp. nov. from the skunk Mephitis mephitis in Quebec. Canadian Journal of Zoology 34: 595-601.

WEBSTER, W. A., AND G. A. CASEY. 1973. Studies on the parasites of Chiropter. III. Helminths from various bat species collected in British Columbia. Canadian Journal of Zoology 51: 633-636.

WHITAKER, J. O., JR., AND R. E. MUMFORD. 1971. Notes on a collection of bats taken by mist-netting at an Indiana cave. American Midland Naturalist 85: 277-279.

WHITLOCK, S. C. 1939. The prevalence of disease and parasites in whitetail deer. Proceedings of the Fourth North American Wildlife Conference 4: 244-249.

WIGGINS, J. P., M. COSGROVE, AND H. ROTHENBACHER. 1980. Gastrointestinal parasites of the eastern cottontail (Sylvilagus floridanus) in central Pennsylvania. Journal of Wildlife Diseases 16: 541-544.

WILLIAMS, R. R. 1960. Acanthatrium lunatum n. sp., a parasite of the big brown bat and a key to the described species of Acanthatrium (Trematoda: Lecithodendriidae). Ohio Journal of Science 60(6): 323-326.

WILLIAMS, R. R. 1960. Acanthatrium lunatum n. sp., a parasite of the big brown bat and a key to the described species of Acanthatrium (Trematoda: Lecithodendriidae). Ohio Journal of Science, 60 (6): 323-326.

- 1961. A bat trematode, Prosthodendrium naviculum Macy, 1936, naturally occurring in raccoons. Journal of Parasitology 47: 640 .

1962. Trematodes from the cavebat, Myotis sodalis Miller and Allen. The Ohio Journal of Science 5: 273.

WILLIAMS, R. R., AND D. G. MYER. 1964. Metagonimoides oregonensis Price, 1931 occurring in Ohio raccoons (Trematoda: Heterophyidae). Ohio Journal of Science 64: 400 .
WITTROCK, D. D. 1976. Structure of the cirrus tegument of Quinqueserialis quinqueserialis (Trematoda: Notocotylidae). Journal of Parasitology 62: 834-836.

WITTROCK, D. D., AND G. L. HENDRICKSON. 1979. Helminths of shrews, Blarina brevicauda and Sorex cinereus in Iowa. Journal of Parasitology 65: 985-986.

WITTROCK, D. D., AND M. J. ULMER. 1974. Helminths of badgers, Taxidea taxus (Schreber), in northwest Iowa. Iowa State Journal of Research 48: 319-327.

WOBESER, G., W. RUNGE, AND R. R. STEWART. 1983. Metorchis conjunctus (Cobbold, 1860) infection in wolves (Canis lupus), with pancreatic involvement in two animals. Journal of Wildlife Diseases 19: 353-356.

WOODARD, J. C., S. G. ZAM, D. K. CALDWELL, AND M. C. CALDWELL. 1969. Some parasitic diseases of dolphins. Pathologia Veterinaria 6: 257-272.

WOODHEAD, A. E., AND H. MALEWITZ. 1936. Mediogonimus ovilacus, n. g., n. sp.. Journal of Parasitology 22: 273-275.

WOOTTON, D. M., AND K. D. MURRELL. 1967. Ptyalincola ondatrae, gen. et sp. $\mathrm{n}$. (Trematoda: Brachylaimidae), a fluke inhabiting the salivary glands of muskrats. Journal of Parasitology 53: 739-742.

WORLEY, D. E., J. C. FOX, J. B. WINTERS, R. H. JACOBSON, AND K. R. GREER. 1976. Helminth and arthropod parasites of grizzly and black bears in Montana and adjacent areas. Papers and Proceeding of the Third International Confonference on Bears, Paper 45, pp 455-463.

ZAJAC, A. M., AND J. F. WILLIAMS. 1980. Infection with Schistosomatium douthitti (Schistosomatidae) in the meadow vole (Microtus pennsylvanicus) in Michigan. Journal of Parasitology 66: 366-367.

ZENCHAK, J. J., AND J. E. HALL. 1971. Helminths from Peromyscus leucopus and $P$ maniculatus in West Virginia. Journal of Parasitology 57: 542.

ZYLL DE JONG, C. G. VAN. 1966. Parasites of the Canada lynx, Felis (Lynx) canadensis (Kerr). Canadian Journal of Zoology 44: 499-509. 


\section{SUPPLEMENTARY \\ READING}

AMEEL, D. J. 1931a. Life history of the North American lung fluke of mammals. Journal of Parasitology 18: 114.

1931b. Life history of the North American lung fluke of mammals. Journal of Parasitology 18: 264-268.

ANDERSON, R. C. 1962. The helminth and arthropod parasites of the white-tailed deer (Odocoileus virginianus): A general review. Transactions of the Royal Canadian Institute 34: 57-92.

BARBER, D. L., AND L. L. LOCKARD. 1973. Some helminths from mink in southwestern Montana, with a checklist of their internal parasites.Great Basin Naturalist 33: 53-60.

BRENES, R. R., R. ZELEDON, AND G. ROJAS. 1968. The findings of Paragonimus sp. in mammals, crabs, and snails in Costa Rica. Boletin Chileno De Parasitologia 23: 164.

BRISTOL, J. R., AND A. G. CANARIS. 1973. Physiology, ecology, and life cycle of Brachylaime microti (Trematoda). American Philosophical Society Year Book 1973: 296-298.

CABALLERO, Y C. E. 1960. Trematodos de los murcielagos de Mexico. VIII. Catalogo taxonomico de los trematodos que parasitan a los murcielagos (Mammalia, Chiroptera Blumenbach, 1774). Anales del Instituto de Biologia. Universidad de Mexico 31: 215287.

CONTI, J. A., D. J. FORRESTER, R. K. FROHLICH, G. L. HOFF, AND W. J. BIGLER. 1984. Helminths of urban gray squirrels in Florida. Journal of Parasitology 70: 143-144.

CORDY, D. R. AND J. R. GORHAM. 1950. The pathology and etiology of salmon disease in the dog and fox. American Journal of Pathology 26:617-637.

CURTICE, C. 1892. Parasites, being a list of those infesting domesticated animals and man in the United States. Journal of Comparative Medicine and Veterinary Archives (1892): 223-236.

DIKMANS, G. 1939. Helminth parasites of North American semidomesticated and wild ruminants. Proceedings of the Helminthological Society of Washington 6: 97-101.
DORAN, D. J. 1955. A catalogue of the Protozoa and helminths of North American rodents. IV. Trematoda. The American Midland Naturalist 53: 446-454.

FOREYT, W. J., AND A. C. TODD. 1979. Selected clinicopathological changes associated with experimentally induced Fascioloides magna infection in whitetailed deer. Journal of Wildlife Diseases 15: 83-89.

GERACI, J. R., AND D. J. STAUBIN. 1987. Effects of parasites on marine mammals. International Journal of Parasitology 17: 407-414.

GOMES, D. C., AND J. J. VICENTE. 1972. Estudo do genero Rhopalias Stiles and Hassall, 1898 (Trematoda, Rhopaliasidae). Memoirs of the Institute of Oswaldo Cruzi 70: 115-133.

HARWOOD, P. D. 1939. Notes on Tennessee helminths. IV. North American trematodes of the subfamily Notocotylinae. Journal of the Tennessee Academy of Science 14: 332340.

KITSNER, T. P., AND L. D. KOLLER. 1975. Experimentally induced Fasciola hepatica infections in black-tailed deer. Journal of Wildlife Diseases 11: 214-220.

KRULL, W. H. 1934. New experimental hosts for Brachylaemus virginiana (Dickerson) Krull. Journal of the Washington Academy of Science 24: 483-485.

LAMOTHE-ARGUMEDO, R. 1985. La paragonimiasis en el continente Americano. Salud Publica del Mexico 27: 514-523.

LAMOTHE-ARGUMEDO, R., AND B. J. CRUZ. 1982. Trematoda-Parasitic stages. In: Aquatic Biota of Mexico, Central America and the West Indies. S. H. Hurlbert and A. Villalobos-Figueroa, eds. San Diego State University, San Diego, California.

LAMOTHE-ARGUMEDO, R., E. A. MALEK, AND O. MEAVE-GALLEGOS. 1983. Aroapyrgus alleei Morrison, 1946 (Gastropoda:Hydrobiidae) first intermediate host of Paragonimus mexicanus in Colima, Mexico. Journal of Parasitology 69: 226-228. 
LAMOTHE-ARGUMEDO, R., AND R. B. MADRIGAL. 1983. Descripcion de una especie nueva de Amphimerus Barker,1911 (Trematoda: Opisthorchidae) de Costa Rica. Anales del Institute de Biologia. Universidad Nal Auton del Mexico 54, Ser Zoologia 1: 13-21.

MAS-COMA, S., J. G. ESTEBAN, AND M. A.

VALERO. 1986. The genus

Scaphiostomum Braun, 1901 (Trematoda:

Brachylaimidae): A systematic review and description of Scaphiostomum palaearcticum n. sp.. Systematic Parasitology 8: 141-150.

MILLEMANN, R. E. AND S. E. KNAPP. 1970.

Biology of Nanophyetus salmincola and "Salmon Poisoning" disease. Advances In Parasitology 8: 1-41.

MIYAZAKI, I. 1964. Notes on the metacercaria of Paragonimus kellicotti Ward, 1908 in North America (Trematoda: Troglotrematidae). Japanese Journal of Parasitology 13: 453457.

MIYAZAKI, I., AND Y. ISHII. 1968.

Comparative study of the Mexican lung flukes with Paragonimus kellicotti Ward, 1908. Journal of Parasitology 54: 845-846.

OLIVIER, L. 1952. A comparison of infections in mice with three species of schistosomes, Schistosoma mansoni, Schistosoma japonicum and Schistosomatium douthitti. American Journal of Hygiene 55: 22-35.

PRICE, E. W. 1953. The fluke situation in American ruminants. Journal of Parasitology 39: 119-134.

REDETZKE, K.A., AND A. G. CANARIS. 1977. Brachylaime microti: A mechanistic simulation model of the parasite, its intermediate snail host, Oreohelix strigosa and its definitive rodent hosts, Paromyscus maniculatus and Microtus montanus. Experimental Parasitology 41: 229-241.
REYNOLDS, B. D. 1938. Metacercariae of a species of Brachylaemus, probably $B$. virginianus, from Agriolimax agrestis. Journal of Parasitology 24: 377-378.

ROGERS, L. L., AND S. M. ROGERS. 1974. Parasites of bears: A review. In bears - their biology and management. M. R. Pelton, J. W. Lentfer, and G. E. Folk eds. The International Union for Conservation of Nature and Natural Resources 40: 411-430.

SAMUEL, W. M. 1968. Endoparasites of domestic ruminants and white-tailed deer.

Transactions of the Thirty-third North American Wildlife and Natural Resources Conference: 364-372.

SHOOP, W. L., AND K. C. CORKUM. 1987. Maternal transmission by Alaria marcianae (Trematoda) and the concept of amphiparatenesis. Journal of Parasitology 73: 110-115.

SIMMS, B. T., C. R. DONHAM, AND J. N. SHAW 1931. Salmon poisoning. American Journal of Hygiene 13: 363-391.

TANABE, B. 1923. The life history of a new schistosome, Schistosomatium pathlocopticum Tanabe, found in experimentally infected mice. Journal of Parasitology 9: 183-198.

ULMER, M. J. 1951. Postharmostomum helicis (Leidy, 1847) Robinson, 1949, (Trematoda), its life history and revision of the subfamily Brachylaeminae. Transactions of the American Microscopical Society 70: 189238. 


\section{INDEX \\ PARASITES}

Acanthatrium alicatai 14

Acanthatrium amphidymum 14

Acanthatrium beuschleini 15

Acanthatrium eptesici 15

Acanthatrium lunatum

(see: Acanthatrium pipistrelli) 15

Acanthatrium microcanthum 15

Acanthatrium nycteridis 15

Acanthatrium oligacanthum 15

Acanthatrium oregonense 15

Acanthatrium pipistrelli 15

Acanthatrium spp. 15

Acanthatrium umbracutatum 15

Alaria alata 24

Alaria americana 24

Alaria arisaemoides 25

Alaria canis

(see: Alaria americana) 24

Alaria canadensis

(see: Alaria mustelae) 25

Alaria craterum 25

Alaria dubia (see: Alaria mustelae) 25

Alaria intermedia (see: Alaria mustelae)25

Alaria marcianae 25

Alaria minnesotae 25

Alaria minuta (see: Alaria mustelae) 25

Alaria mustelae 25

Alaria nasuae 26

Alaria oregonensis (see: Alaria arisaemoides) 25

Alaria spp. 26

Alaria taxideae (see: Alaria mustelae) 25

Allassogonoporidae 13

Allassogonoporus marginalis 13

Allassogonoporus vespertilionis 13

Amphimerus neotropicalis 9

Amphimerus pseudofelineus 9

Amphistoma fabaceum (see: Chiorchis fabaceus) 12

Amphistomum subfriquetrum

(see: Stichorchis subtriquetrus) 12

Anenterotrematidae 13

Anenterotrema freitasi 13

Apophallus spp. 7

Apophallus venustus 7

Apophallus zalophi

(see: Pricetrema zalophi) 9
Ascocotyle ampullacea 7

Ascocotyle diminuta

(see: Phagicola diminuta) 8

Ascocotyle leighi 7

Ascocotyle longa (see: Phagicola longa) 8

Ascocotyle nana (see: Phagicola nana) 8

Ascocotyle pachycystis 7

Baschkirovitrema incrassatum 3

Brachylaemidae 22

Brachylaemus bravoae (see: Brachylaima bravoae) 22

Brachylaemus migrans (see: Brachylaima virginiana) 23

Brachylaima bravoae 22

Brachylaima chiapensis 22

Brachylaima didelphus 22

Brachylaima dolichodirus 23

Brachylaima opisthotrias 23

Brachylaima opisthotrias virginiana 23

Brachylaima ovatus 23

Brachylaima peromysci 23

Brachylaima rauschi 23

Brachylaima rhomboideus 23

Brachylaima spp. 23

Brachylaima thompsoni 23

Brachylaima virginiana 23

Brachylaime microti (see: Scaphiostomum microti) 24

Brachylaimus virginianus (see: Brachylaima virginiana) 23

Brachylecithum spp. 13

Braunina cordiformis 29

Calicophoran microbothrioides 12

Campula oblongata 2

Campula palliata 2

Campula rochebruni 2

Campulidae 2

Carneophallus turgidus 18

Castroia silvai 15

Catatropis filamentis

(see: Notocotylus filamentis) 10

Catatropis fimbriata 10

Catatropis johnstoni 10

Cephalophallus obscurus 13

Cercaria szidati

(see: Linstowiella szidati) 24

Chiorchis fabaceus 12

Conspicuum icteridorum 13 
Cotylophallus similis 7

Cotylophallus venustus

(see: Apophallus venustus) 7

Cryptocotyle concavum 7

Cryptocotyle jejuna 7

Cryptocotyle lingua 7

Cyathocotylidae 24

Dicrocoeliidae 13

Dicrocoelium dendriticum 13

Dicrocoelium lasiuri 13

Dicrocoelium rileyi 14

Dictyonograptus chamelensis 14

Didelphodiplostomum variabile 26

Digenea 2

Digenea Unidentified 29

Diplostomidae 24

Diplostomum mergi 26

Diplostomum variabile

(see: Didelphodiplostomum variabile) 26

Distoma helicis

(see: Postharmostomum helicis) 24

Distoma magnum

(see: Fascioloides magna) 5

Distoma recurvatum

(see: Echinoparyphium recurvatum) 3

Distoma tricolor

(see: Hasstilesia tricolor) 27

Distomum echinatum

(see:Echinostoma echinatum) 3

Distomum gastrophilum

(see: Pholeter gastrophilus) 8

Distomum limatulum

(see: Limatulum limatulum) 16

Distomum lingua

(see: Cryptocotyle lingua) 7

Distomum opisthotrias

(see: Brachylaima opisthotrias) 23

Distomum palliatum

(see: Campula palliata) 2

Distomum rochebruni

(see: Campula rochebruni) 2

Distomum tubiporum

(see: Maxbraunium tubiporum) 16

Echinochasmus schwartzi 3

Echinoparyphium contiguum 3

Echinoparyphium recurvatum 3

Echinoparyphium spp. 3

Echinostoma callawayensis 3

Echinostoma echinatum 3

Echinostoma revolutum 3

Echinostoma spp. 4

Echinostomatidae 3

Echinostomatiformes 2

Echinostomum armigerum

(see: Echinostoma revolutum) 3
Echinostomum callawayensis

(see: Echinostoma callawayensis) 3

Echinostomum coalitum

(see: Echinostoma revolutum) 3

Echinostomum inerme

(See: Euparyphium inerme) 4

Echinostomum sp.

(see: Echinostoma spp.) 4

Ectosiphonus ovatus

(see: Brachylaima ovatus) 23

Enhydridiplostomum alarioides 26

Entosiphonus thompsoni

(see: Brachylaima thompsoni) 23

Euparyphium beaveri 4

Euparyphium inerme 4

Euparyphium melis 4

Euparyphium spp. 4

Euryhelmis monorchis 7

Euryhelmis pacificus 8

Euryhelmis pyriformis 8

Euryhelmis squamula 8

Eurytrema allentoshi 14

Eurytrema komareki 14

Eurytrema procyonis 14

Eurytrema vulpis

(see: Eurytrema procyonis) 14

Fasciola hepatica 4

Fasciola magna

(see: Fascioloides magna) 5

Fasciolidae 4

Fascioloides magna 5

Fibricola cratera 26

Fibricola laruei

(see: Fibricola cratera) 26

Fibricola lucida 27

Fibricola nana

(see: Fibricola cratera) 26

Fibricola spp. 27

Fibricola texensis 27

Glyptoporus noctophilus 15

Gynaecotyla adunca 18

Gyrabascus brevigastrus 15

Gyrosoma singularis 6

Hadwenius mironovus 2

Hadwenius nipponicus 2

Hadwenius seymouri 2

Harmostomum opisthotrias

(see: Brachylaima opisthotrias) 23

Harmostomum opisthotrias virginiana

(see: Brachylaima opisthotrias virginiana) 23

Hasstilesia novicia

(see: Hasstilesia tricolor) 27

Hasstilesia spp. 28

Hasstilesia texensis

(see: Hasstilesia tricolor) 27 
Hasstilesia tricolor 27

Hasstilesiidae 27

Hemistomum craterum (see: Fibricola cratera) 26

Heterobilharzia americana 28

Heterobilharzia spp. 28

Heterophyidae 7

Ityogonimus scalopi 24

Lecithodendriidae 14

Lecithodendrium anticum 15

Lecithodendrium breckenridge (see: Ochoterenatrema breckenridgei) 16

Lecithodendrium posticum 15

Lecithodendrium spp. 16

Lecithodesmus goliath 2

Lecithodesmus spinosus 2

Leucasiella mironovi (see: Hadwenius mironovus) 2

Leucochloridiomorphidae 28

Limatulum costarricensis 16

Limatulum diminutum (see: Ochoterenatrema diminutum) 16

Limatulum gastroides 16

Limatulum istmicus 16

Limatulum limatulum 16

Limatulum mcdanieli 16

Limatulum oklahomensis 16

Limatulum scabrum (see: Prosthodendrium naviculum) 17

Limatulum spp. 16

Linstowiella szidati 24

Lyperosomum intermedium 14

Lyperosomum sinuosum 14

Maritreminoides nettae 18

Maritrema prolixum 18

Maritreminoides spp.19

Maxbraunium tubiporum 16

Mediogonimus ovilacus 21

Metagonimoides oregonensis 8

Metorchis conjunctus 9

Microphallidae 18

Microphallus aspalacis 19

Microphallus enhydrae 19

Microphallus pirum 19

Microphallus spp. 19

Monostomum affinis 12

Myotitrema asymmetrica 13

Nanophyetidae 19

Nanophyetus salmincola 19

Nanophyes salmincola

(see: Nanophyetus salmincola) 19

Nasitrema dalli 5

Nasitrema delphini 5
Nasitrema globicephalae 5

Nasitrema spp. 6

Nasitrema stenosoma 5

Nasitrematidae 5

Neodiplostomum lucidum (see: Fibricola lucida) 27

Neoglyphe locellus 20

Neoglyphe soricis 20

Notocotyle quinqueseriale

(see: Quinqueserialis quinqueserialis) 11

Notocotylidae 10

Notocotylus filamentis 10

Notocotylus hassalli 10

Notocotylus spp. 10

Notocotylus urbanensis 10

Nudacotyle novica 10

Nudacotyle spp. 10

Ochoterenatrema breckenridgei 16

Ochoterenatrema caballeroi (see: Ochoterenatrema diminutum) 16

Ochoterenatrema diminutum 16

Ochoterenatrema labda 16

Ochoterenatrema travassosi 17

Odhneriella seymouri

(see: Hadwenius seymouri) 2

Ogmogaster antarctica 10

Ogmogaster pentalineatus 11

Ogmogaster plicatus 11

Ogmogaster trilineatus 11

Opisthioglyphe locellus

(See Neoglyphe locellus) 20

Opisthorchiidae 9

Opisthorchiiformes 7

Opisthorchis pricei 9

Opisthorchis tenuicollis 9

Opisthorchis tonkae 9

Orthosplanchnus arcticus 2

Orthosplanchnus fraterculus 2

Ototrema schildti 17

Panopistus pricei 24

Paragonimidae 19

Paragonimus ecuadoriensis

(see: Paragonimus mexicanus) 19

Paragonimus inca (see: Paragonimus mexicanus) 19

Paragonimus kellicotti 19

Paragonimus mexicanus 19

Paragonimus peruvianus 19

(see: Paragonimus mexicanus) 19

Paragonimus rudis 20

Paragonimus spp. 20

Paragonimus westermani 20

Paralecithodendrium carlsbadensis 17

Paralecithodendrium chilostomum 17

Paralecithodendrium emollidum 17 
Paralecithodendrium macnabi 17

Paralecithodendrium naviculum 17

Paralecithodendrium nokomis 17

Paralecithodendrium swansoni 17

Paralecithodendrium transversum 18

Parallelorchis diglossus 27

Parametorchis canadensis 9

Parametorchis complexus 9

Parametorchis spp. 10

Paramonostomum pseudalveatum 11

Paramphistomidae 12

Paramphistomiformes 10

Paramphistomum castori

(see: Stichorchis subtriquetrus) 12

Paramphistomum cervi 12

Paramphistomum liorchis 12

Paramphistomum microbothrioides

(see: Calicophoron microbothrioides) 12

Paramphistomum spp.12

Parascocotyle lageniformis

(see: Phagicola diminuta) 12

Phagicola diminuta 8

Phagicola lageniformis

(see: Phagicola diminuta) 8

Phagicola longa 8

Phagicola nana 8

Pharyngostomoides adenocephala 27

Pharyngostomoides ovale 27

Pharyngostomoides procyonis 27

Phocitrema fusiforme 8

Pholeter gastrophilus 8

Plagiorchiidae 20

Plagiorchiiformes 13

Plagiorchis elegans 20

Plagiorchis massino 20

Plagiorchis micracanthos 20

Plagiorchis micracanthum

(see: Plagiorchis micracanthos) 20

Plagiorchis muris 21

Plagiorchis noblei 21

Plagiorchis proximus 21

Plagiorchis spp. 21

Plagiorchis vespertilionis 21

Plagiorchis vespertilionis parorchis 21

Platynosomum allentoshi

(see: Eurytrema allentoshi) 14

Platynosomum beltrani 14

Postharmostomum helicis 24

Postharmostomum laruei

(see: Postharmostomum helicis) 24

Postharmostomum noveboracensis 24

Pricetrema eumetopii 9

Pricetrema phocae 9

Pricetrema spp. 9

Pricetrema zalophi 9

Proalaria variabilis

(see: Didelphodiplostomum variabile) 26
Procyotrema marsupiformis 27

Prosthodendrium alaskensis 18

Prosthodendrium diminutum (see: Ochoterenatrema diminutum) 16

Prosthodendrium duboisi (see: Paralecithodendrium macnabi) 17

Prosthodendrium emollidum (see: Paralecithodendrium emollidum) 17

Prosthodendrium longiforme 18

Prosthodendrium macnabi (see: Paralecithodendrium macnabi) 17

Prosthodendrium mizellei (see: Paralecithodendrium macnabi) 17

Prosthodendrium naviculum (see: Paralecithodendrium naviculum) 17

Prosthodendrium nokomis (see: Paralecithodendrium nokomis) 17

Prosthodendrium oligolecithum (see: Paralecithodendrium chilostomum) 17

Prosthodendrium paeminosum (see: Paralecithodendrium naviculum) 17

Prosthodendrium scabrum (see: Paralecithodendrium naviculum) 17

Prosthodendrium silvai 18

Prosthodendrium singularium 18

Prosthodendrium spp. 18

Prosthodendrium swansoni (see: Paralecithodendrium swansoni) 17

Prosthodendrium tetralobulatum (see: Paralecithodendrium naviculum) 17

Prosthodendrium transversum (see: Paralecithodendrium transversum) 18

Prosthodendrium travassosi (see: Ochoterenatrema travassosi)54 17

Prosthodendrium volaticum (see: Paralecithodendrium transversum) 18

Prosthogonimidae 21

Pseudodiscus zibethicus (see: Wardius zibethicus) 12

Pseudomaritrema beolopolskoi 19

Pseudospelotrematoides spp. 19

Psilostomidae 6

Psilostomum ondatrae 6

Psilostomum spp. 6

Psilotrema spp. 6

Ptyalincola ondatrae 28

Quinqueserialis floridensis 11

Quinqueserialis hassalli 11

Quinqueserialis quinqueserialis 11

Quinqueserialis spp. 11

Quinqueserialis zibethicai 11

Quiscalus spp.

(see: Conspicuum icteridorum) 13

Rhopalias baculifer 6

Rhopalias coronatus 6 
Rhopalias horridus 6

Rhopalias macracanthus 6

Rhopaliasidae 6

Ribeiroia ondatrae 6

Roberidigenea dollfusi 14

Rossicotrema venustus

(see: Apophallus venustus) 7

Scaphiostomum microti 24

Scaphiostomum pancreaticum 24

Schistosomatidae 28

Schistosomatium douthitti 28

Sellacotyle mustelae

(see: Troglotrema mustelae) 22

Sellacotyle vitellosa 22

Stephanoproraoides lawi 6

Stephanoprora spinosa 4

Stichorchis subtriquetrus 12

Strigeiformes 22

Synthesium tursionis 2
Telorchiidae 22

Telorchis spp. 22

Tremajoannes buckleyi 18

Trematoda 2

Troglotrematidae 22

Troglotrema mustelae 22

Urotrematidae 22

Urotrema scabridum 22

Urotrema shillingeri 22

Wardius zibethicus 12

Xiphidiotrema lockerae 22

Zalophotrema hepaticum 2

Zalophotrema spp. 3

Zonorchis komareki 14

Zygocotyle lunata 13 
Aello megalophylla 37

Alces alces 31

Alces alces americana 31

Alces americanus

(see: Alces alces americana) 31

Alopex lagopus 32

Artiodactyla 31

Balaenoptera borealis 36

Balaenoptera physalus 36

Balaenoptera sp. 36

Balaenopteridae 36

Balantiopteryx ochoterenai

(See:Balantiopteryx plicata) 37

Balantiopteryx plicata 37

Bison bison 31

Blarina brevicauda 40

Blarina brevicauda brevicauda 40

Blarina brevicauda kirtlandi 40

Bos taurus 31

Bovide 31

Callorhinus ursinus 41

Caluromys derbianus pallidus 46

Canidae 32

Canis latrans 32

Canis latrans lestes 32

Canis lestes

(see: Canis latrans lestes) 32

Canis lupus 32

Canis rufus 32

Canis rufus $\times$ Canis latrans 32

Capromyidae 42

Cariacus virginianus

(see: Odocoileus virginianus) 31

Carnivora 32

Castor canadensis 42

Castor canadensis carolinensis 42

Castor canadensis leucodontos 42

Castor canadensis mexicanus 42

Castoridae 42

Cervidae 31

Cervus elaphus 31

Cervus elaphus nelsoni 31

Cervus canadensis nelsoni

(see: Cervus elaphus nelsoni) 31

Cervus elaphus roosevelti 31

Cervus canadensis roosevelti

(see: Cervus elaphus roosevelti) 31

Cervus unicolor 31

Cetacea 36

Chilonycteris rubiginosus
(see:Pteronotus parnellii rubiginosus) 38

Chiroptera 37

Clethrionomys gapperi 42

Clethrionomys rutilus dawsoni 42

Cottontail 41

Dasypodidae 40

Dasypterus floridanus

(see: Nycteris intermedia floridana) 39

Dasypus novemcinctus 40

Delphinapterus leucas 37

Delphinidae 36

Delphinus delphis 36

Delphinus bairdi

(see: Delphinus delphis) 36

Dicotyles tajacu angulatus 32

Dicrostonyx groenlandicus rubricatus 42

Didelphidae 46

Didelphis marsupialis 46

Didelphis marsupialis caucae 46

Didelphis marsupialis etensis (see:Didelphis marsupialis caucae) 46

Didelphis marsupialis mesamericana (see: Didelphis virginiana californica) 46

Didelphis marsupialis tabascensis 46

Didelphis marsupialis virginiana (see: Didelphis virginiana virginiana) 46

Didelphis mesamericana mesamericana (see: Didelphis virginiana) 46

Didelphis mesamericana tabascensis (see: Didelphis marsupialis tabascensis) 46

Didelphis spp. 46

Didelphis virginana 46

Didelphis virginiana californica 46

Didelphis virginiana virginiana 46

Edentata 40

Emballonuridae 37

Enhydra lutris 33

Eptesicus fuscus 38

Eptesicus fuscus fuscus 38

Eptesicus nilssoni 38

Eptesicus propinguus 40

Erethizon dorsatum 42

Erethizontidae 42

Erignathus barbatus 42

Eschrichtidae 37

Eschrichtius gibbosus 37

Eschrichtius robustus

(see: Eschrichtius gibbosus) 37

Eumetopias jubatus 41

Eutheria 31 
Felidae 33

Felis concolor 33

Felis rufa

(see: Lynx rufus) 33

Felis onca 33

Felis pardalis 33

Geomyidae 42

Glaucomys sabrinus 45

Globicephala melaena 36

Globicephala sieboldii 36

Gulo gulo (see: Gulo luscus) 33

Gulo luscus 33

Heteromyidae 42

Insectivora 40

Jack rabbit 41

Lagenorhynchus obliquidens 36

Lagomorpha 41

Lasionycteris noctivagans 38

Lasiurus borealis

(see: Nycteris borealis) 39

Lasiurus borealis borealis

(see: Nycteris borealis borealis) 39

Lasiurus cinereus

(see: Nycteris cinerea) 39

Leporidae 41

Lepus americanus 41

Lepus americanus phaeonotus 41

Lepus americanus struthopus 41

Lepus californicus merriami 41

Lepus californicus texianus 41

Lepus sylvaticus

(see: Sylvilagus nuttallii) 41

Lepus texianus

(see: Lepus californicus texianus) 41

Liomys pictus pictus 42

Lissodelphis borealis 36

Lutra canadensis 33

Lutra canadensis vaga

(see: Lutra canadensis) 33

Lynx canadensis 33

Lynx rufus 33

Lynx rufus fasciatus 33

Lynx fasciatus fasciatus

(see: Lynx rufus fasciatus) 33

Lynx rufus floridanus 33

Mammalia 31

Manatus latirostris

(see: Trichechus manatus) 46

Marmosa mexicana mexicana 47

Marmota monax 45

Marmota monax rufescens 45
Marsupialia 46

Martes americana 33

Martes americana actuosa 33

Martes pennanti 33

Mephitis macroura macroura 33

Mephitis mephitis 33

Mephitis mephitis avia 34

Mephitis mephitis nigra 34

Mephitis nigra

(see: Mephitis mephitis nigra) 34

Metachirops opossum 47

Metachirops opossum fuscogriseus 47

Metachirops opossum pallidus 47

Metatheria 46

Micronycteria hirsuta 37

Microtus longicaudus baileyi 43

Microtus montanus caryi (see: Microtus montanus nanus) 43

Microtus montanus fusus 43

Microtus montanus nanus 43

Microtus miurus muriei 43

Microtus miurus paneaki

(see: Microtus miurus muriei) 43

Microtus oeconomus macfarlani 43

Microtus pennsylvanicus 43

Microtus pennsylvanicus drummondii 43

Microtus pennsylvanicus modestus 43

Microtus pennsylvanicus pennsylvanicus 43

Microtus pennsylvanicus terraenovae 43

Mirounga angustirostris 42

Molossidae 37

Monodontidae 37

Mormoops megalophylla (see: Aello megalophylla) 37

Mormoops megalophylla senicula (see: Aello megalophylla) 37

Muridae 42

Mus musculus 43

Mustela cicognanii (see: Mustela erminea cicognanii) 34

Mustela erminea 34

Mustela erminea cicognanii 34

Mustela frenata 34

Mustela frenata noveboracensis 34

Mustela noveboracensis noveboracensis (see: Mustela frenata noveboracensis) 34

Mustela spp. 34

Mustela vison 34

Mustela vison letifera 34

Mustela vison vulgivaga 34

Mustelidae 33

Myocastor coypus 42

Myocastor coypus bonariensis 42

Myotis californicus 38

Myotis californicus caurinus 38

Myotis evotis evotis 38

Myotis grisescens 38 
Myotis keenii 38

Myotis lucifugus 38

Myotis lucifugus alascensis 39

Myotis lucifugus lucifugus 39

Myotis lucifugus occultus 39

Myotis nigricans nigricans 39

Myotis sodalis 39

Myotis velifer incautus 39

Myotis volans longicrus 39

Nasua narica

(see: Nasua nasua) 35

Nasua nasua 35

Natalidae 37

Natalus stramineus mexicanus 37

Natalus mexicanus

(see: Natalus stramineus mexicanus) 37

Neofiber alleni nigrescens 43

Neurotrichus gibbsii 40

Nycteris borealis 39

Nycteris borealis borealis 39

Nycteris cinerea 39

Nycteris intermedia floridana 39

Nycticeius humeralis 39

\section{Ochotona collaris 41}

Ochotonidae 41

Odocoileus hemionus 31

Odocoileus hemionus columbianus 31

Odocoileus hemionus hemionus 31

Odocoileus spp. 31

Odocoileus virginianus 31

Odocoileus virginianus borealis 31

Odocoileus virginianus leucurus 31

Ondatra rivalicia

(see: Ondatra zibethicus rivalicius) 44

Ondatra zibethicus 43

Ondatra zibethicus macrodon 44

Ondatra zibethicus obscurus 44

Ondatra zibethica occipitalis 44

Ondatra zibethicus osoyoosensis 44

Ondatra zibethicus rivalicius 44

Ondatra zibethicus zibethicus 44

Oryzomys palustris 44

Otariidae 41

Panthera onca

(see: Felis onca) 33

Peromyscus banderanus banderanus 44

Peromyscus gossypinus gossypinus 44

Peromyscus guatemalensis guatemalensis 44

Peromyscus leucopus 44

Peromyscus leucopus leucopus 45

Peromyscus leucopus noveboracensis 45

Peromyscus maniculatus 45

Peromyscus maniculatus bairdii 45

Peromyscus maniculatus sonoriensis 45

Philander laniger pallidus (see: Caluromys derbianus pallidus) 46

Philander opossum

(see: Metachirops opossum) 47

Philander opossum fuscogriseus

(see: Metachirops opossum fuscogriseus) 47

Philander opossum pallidus

(see: Metachirops opossum pallidus) 47

Phoca hispida 42

Phoca largha

(see: Phoca vitulina largha) 42

Phoca vitulina 42

Phoca vitulina largha 42

Phoca vitulina richardsii 42

Phocidae 42

Phocoena phocoena 36

Phocaena vomerina

(see: Phocoena phocoena) 36

Phocoenoides dalli 36

Phyllostomatidae 37

Pinnipedia 41

Pipistrellus hesperus 40

Pipistrellus sp. 40

Pipistrellus subflavus 40

Pipistrellus subflavus subflavus 40

Procyonidae 35

Procyon lotor 35

Procyon lotor crassidens 35

Procuon lotor hirtus 35

Procyon lotor litoreus 35

Procyon lotor lotor 35

Procyon lotor pacificus 35

Procyon lotor solutus 36

Procyon psora pacifica

(see: Procyon lotor pacificus) 35

Pteronotus parnellii rubiginosus 38

Phoca hispida 42

Phoca largha

(see: Phoca vitulina largha) 42

Phoca vitulina 42

Phoca vitulina largha 42

Phoca vitulina richardsii 42

Phocidae 42

Phocoena phocoena 36

Phocaena vomerina

(see: Phocoena phocoena) 36

Phocoenoides dalli 36

Phyllostomatidae 37

Pinnipedia 41

Pipistrellus hesperus 40

Pipistrellus sp. 40

Pipistrellus subflavus 40

Pipistrellus subflavus subflavus 40

Procyonidae 35

Procyon lotor 35

Procyon lotor crassidens 35

Procyon lotor hirtus 35

Procyon lotor litoreus 35 
Procyon lotor lotor 35

Procyon lotor pacificus 35

Procyon lotor solutus 36

Procyn psora pacifica

(see: Procyon lotor pacificus) 35

Pteronotus parnellii rubiginosus 38

Rabbit 41

Rangifer caribou sylvestris (see: Rangifer tarandus caribou) 31

Rangifer tarandus caribou 31

Rattus norvegicus 45

Rattus spp. 45

Red bat 40

Reithrodontomys humulis 45

Rodentia 42

Scalopus aquaticus 40

Sciuridae 45

Sciurus carolinensis 45

Sciurus carolinensis carolinensis 45

Sigmodon hispidus 45

Sirenia 46

Shrew 40

Skunk 35

Sorex bendirii palmeri 40

Sorex cinereus 40

Sorex cinereus cinereus 40

Sorex obscurus permiliensis

(see: Sorex vagrans permiliensis) 40

Sorex palustris 40

Sorex palustris navigator 40

Sorex vagrans 40

Sorex vagrans permiliensis 40

Soricidae 40

Spermophilus franklinii 45

Spermophilus richardsonii 45

Spermophilus variegatus utah 45

Spilogale putorius 34

Steno bredanensis 36

Suidae 31

Sus scrofa 31

Sylvilagus aquaticus 41

Sylvilagus aquaticus aquaticus 41

Sylvilagus floridanus 41

Sylvilagus floridanus alacer 41

Sylvilagus floridanus mallurus 41

Sylvilagus floridanus mearnsii 41

Sylvilagus nuttallii 41
Sylvilagus palustris 41

Sylvilagus palustris palustris 41

Sylvilagus transitionalis 41

Tadarida brasiliensis 37

Tadarida brasiliensis cynocephala 37

Tadarida cynocephala

(see: Tadarida brasiliensis cyocnephala) 37

Tadarida brasiliensis mexicana 37

Talpidae 40

Tamiasciurus hudsonicus 45

Tamiasciurus hudsonicus loquax 45

Tamias striatus 45

Tamias striatus lysteri 45

Taxidea taxus 34

Tayassuidae 32

Theria 31

Thomomys talpoides 42

Trichechidae 46

Trichechus manatus 46

Tursiops gillii 36

Tursiops nesarnack 37

Tursiops truncatus (see: Tursiops nesarnack) 37

Urocyon cinereoargenteus 32

Urocyon cinereoargenteus cinereoargenteus 32

Ursidae 36

Ursus americanus 36

Ursus arctos 36

Varying hare 41

Vespertilio lasiurus

(see: Nycteris borealis borealis) 39

Vespertilionidae 38

Vulpes fulva (see: Vulpes vulpes) 32

Vulpes fulva alascensis (see: Vulpes fulva alascensis) 32

Vulpes lagopus (see: Alopex lagopus) 32

Vulpes vulpes 32

Vulpes vulpes alascensis 32

Zalophus californianus 42

Zapodidae 45

Zapus hudsonius 45

Zapus hudsonius hudsonius 46 


